\title{
When Friends Give Bad Advice
}

\author{
Analyzing Response to Recommendation Performance \\ of Close Others
}

\author{
Dissertation \\ zur Erlangung des Doktorgrades \\ der Wirtschaftswissenschaftlichen Fakultät \\ der Georg-August-Universität Göttingen \\ (Dr. rer. pol.)
}

vorgelegt von

Carsten Leo Demming, M.Sc.

geboren in Rhede, Westfalen

Göttingen, 2020 
This page is intentionally left blank. 


\section{Betreuungsausschuss und Prüfungskommission}

Erstgutachterin:

Prof. Dr. Yasemin Boztuğ, Professur für Marketing und Konsumentenverhalten

Georg-August-Universität Göttingen, Platz der Göttinger Sieben 3, 37073 Göttingen

Zweitgutachter:

Prof. Dr. Maik Hammerschmidt, Professur für Marketing und Innovationsmanagement

Georg-August-Universität Göttingen, Platz der Göttinger Sieben 3, 37073 Göttingen

Drittgutachter:

Prof. Dr. Waldemar Toporowski, Professur für Marketing und Handelsmanagement

Georg-August-Universität Göttingen, Platz der Göttinger Sieben 3, 37073 Göttingen

Tag der mündlichen Prüfung: 27.03.2020 
This page is intentionally left blank. 


\section{Acknowledgements}

This work is dedicated to my wife, friend and love, Frauke, who has supported and encouraged me during all the challenges of this process. I am truly thankful for having you in my life.

I also thank my children and my parents for being supportive and forbearing for the last years.

Lastly, I thank my three supervisors and my colleagues for their support and their companionship. It has been a pleasure to work with you. 
This page is intentionally left blank. 


\section{Table of Contents}

List of Figures ..................................................................................................................................... III

List of Tables...................................................................................................................

$1 \quad$ General Introduction................................................................................................... 5

Conceptualization of Recommendations .......................................... 7

1.2 Research on Recommender Evaluation ...........................................

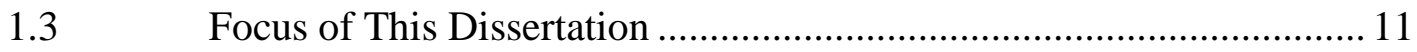

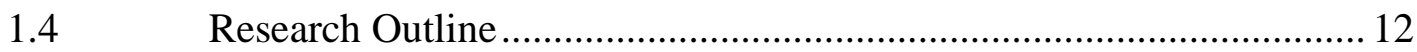

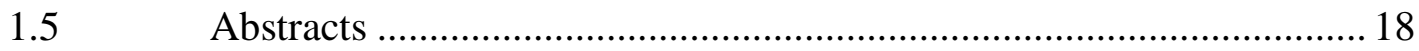

2 Following Fallible Friends: Relationship Closeness Determines How Consumers

Respond to Recent Recommendation Performance (Paper 1) ................................... 21

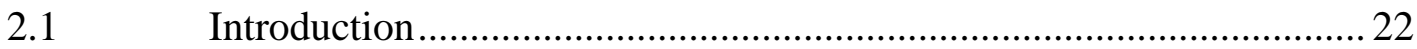

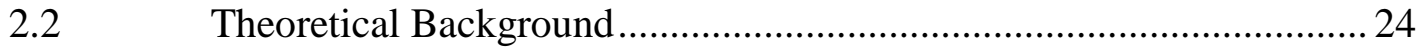

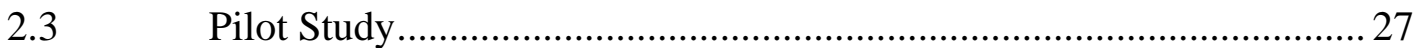

2.4 Main Study: Movie Recommendation ............................................... 29

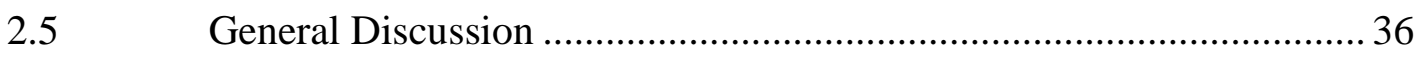

3 Easing a Rough Start: How Relationships to Recommenders Affect Response to

Service Failure in Initial Encounters (Paper 2) ................................................................ 41

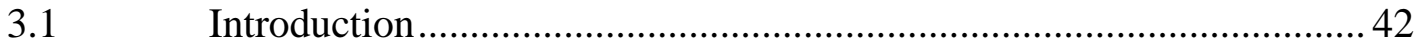

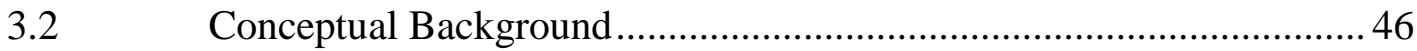

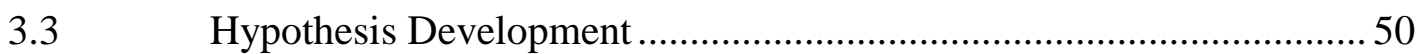

Overview of Studies..................................................................... 53

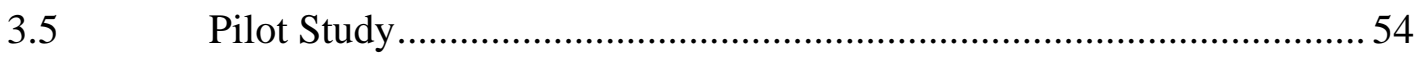


3.6 Study 1: Service Failure at a Doctor's Appointment ...........................56

3.7 Study 2: Service Failure in a Restaurant ...........................................62 62

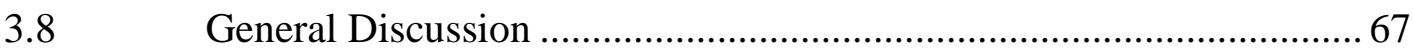

4 Conducting Mediation Analysis in Marketing Research (Paper 3) ....................... 73

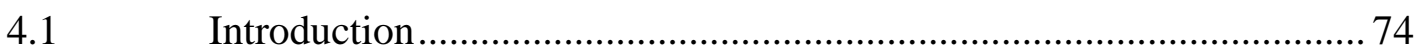

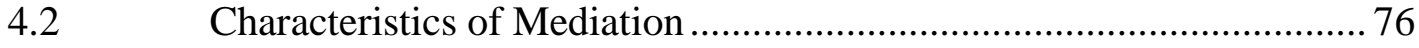

4.3 Approaches to Examine Mediation................................................. 87

4.4 Tutorial: Estimating Mediation Models with PROCESS …................. 93

4.5 Robustness Assessment and Complex Model Testing....................... 108

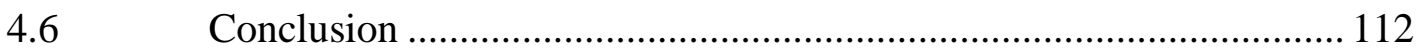

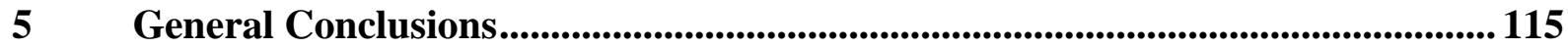

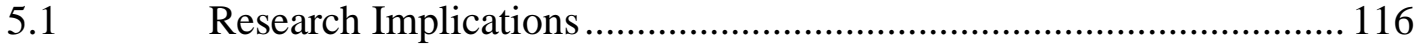

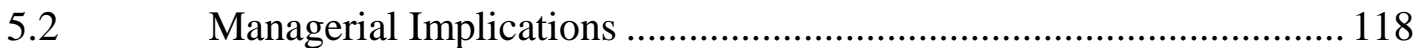

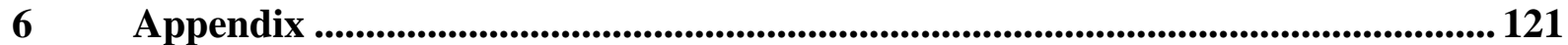

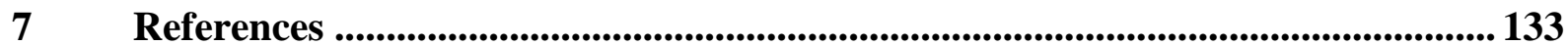




\section{List of Figures}

Figure 1: Dissertation Framework.

Figure 2: Effect of Recent Recommendation Performance on Intent to Follow Friends and

Acquaintances Again.

Figure 3: Effect of Recent Recommendation Performance by Initial Levels of Intent to

Follow.

Figure 4: Effects of Recommendations from Distant and Close Others on Loyalty after IESF.

Figure 5: Belief in Friends and Acquaintances as Recommenders. 55

Figure 6: Citation Trend of Influential Mediation Analysis Publications.............................. 76

Figure 7: Typology of Mediation Model Groups........................................................... 78

Figure 8: Simple Mediation Model as an Extension of a Simple Causal Relationship. .......... 79

Figure 9: Parallel Mediation Model with Two Mediators................................................... 80

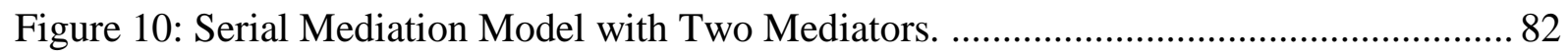

Figure 11: Selection of Variants of a Moderated Mediation Model. ..................................... 84

Figure 12: Mediation Types and Their Implications for Theory Building............................ 86

Figure 13: Example of a Bootstrapped Sampling Distribution of the Indirect Effect $a b \ldots . . . . . .91$

Figure 14: Screenshot of the PROCESS Graphical User Interface in IBM SPSS Statistics.... 94

Figure 15: Simple Mediation Example (PROCESS Model 4)........................................... 95

Figure 16: PROCESS Output for Simple Mediation Example (Model 4). ........................... 97

Figure 17: Parallel Mediation Example (PROCESS Model 4)......................................... 99

Figure 18: PROCESS Output Summary of Parallel Mediation Example (Model 4)............. 101

Figure 19: Serial Mediation Example (PROCESS Model 6)............................................ 102

Figure 20: PROCESS Output Summary of Serial Mediation Example (Model 6)................ 103

Figure 21: Moderated Mediation Example (PROCESS Model 7) ...................................... 104

Figure 22: PROCESS Output Summary of Moderated Mediation Example (Model 7)........ 105 


\section{List of Tables}

Table 1: Overview of the Papers. .................................................................................. 15

Table 2: Data, Sample, Research Context and Methodology of the Empirical Papers............ 17

Table 3: Effect of Way of Acquisition on Intent to Retain a New Practice After Initial

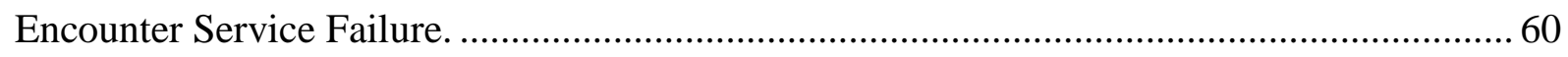

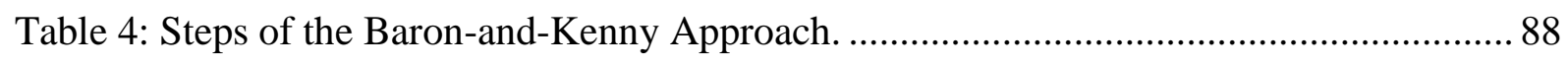

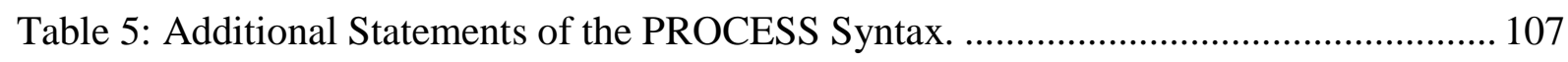




\section{General Introduction}

"The advice of friends must be received with a judicious reserve; we must not give ourselves up to it and follow it blindly, whether right or wrong."

Pierre Charron, Philosopher (1541-1603)

Recommendations are common in everyday interactions of consumers and strongly impact consumption decisions (Chen \& Kirmani, 2015; Urbany, Dickson, \& Wilkie, 1989). Thus, understanding the driving forces that make consumers follow recommendations is equally relevant for managers and consumer researchers. While following good recommendations means that people consume products that satisfy their wants, following bad recommendations leads to poor product decisions and ultimately reduces well-being. Therefore, choosing wisely which recommendations to follow and which not to follow is important (Gershoff, Broniarczyk, $\&$ West, 2001). Relational bonds between the person that recommends and the receiver of the recommendation have been identified as a crucial driver of following recommendations. Thus, the most influential recommendations come straight from the people we know well. According to a global Nielsen survey, $83 \%$ of all interviewed consumers state that they regularly follow the recommendations of friends and family (Nielsen, 2015). This strong reliance on close other's advice is at least partly based on the belief that people from the nearest social surrounding know preferences best (Gershoff \& Johar, 2006). However, in contrast to this lay theory, friends and family members do not necessarily recommend more accurately than more distant recommenders (Eggleston et al., 2015). Consequently, their ability to recommend tends to be overstated (Gershoff \& Johar, 2006; Lerouge \& Warlop, 2006). Similarly, research has shown that close relationships do not necessarily improve ability to predict the relation partner's 


\section{Chapter 1}

preferences (Kenny \& Acitelli, 2001). Frequently, close others recommend what they prefer themselves instead of taking into consideration the preferences of the recommendation receiver (Lerouge \& Warlop, 2006). This is partly due to the fact that they overestimate the degree of their preference similarity to friends or family members (Jussim \& Osgood, 1989; Locke, Craig, Baik, \& Gohil, 2012). In short, it is highly conceivable that not all recommendations from close others result in favorable outcomes that match the preferences of the recommendation receiver. At the same time, prior research suggests the bad recommendations of close others may be inconsistent to consumer beliefs (Eggleston, Wilson, Lee, \& Gilbert, 2015) which may result in discounting negative information. However, blindly relying on recommendations of close others entails potential risks of subsequent bad product decisions. Surprisingly, despite its relevance for many recommendation situations, to our knowledge no prior research has explored the behavioral consequences of conflicting information about recommendation performance and the recommender characteristic relationship closeness. In this dissertation, we address the research gap in the field of recommender evaluation. We investigate the impact of relationship closeness on the response to recommendation outcomes and its consequences for recommenders and firms. Thereby, we especially focus on response to unfavorable recommendation outcomes. This leads to the following research questions:

How do consumers respond when recommendations of close others turn out bad? How does relationship closeness to recommenders shape the reasoning about these negative outcomes? And what are the consequences of this reasoning for recommenders and companies in subsequent decisions?

Our premise is that given the relationship to a recommender is close, this may alter individuals' reasoning about bad recommendation performances of this recommender. Whether consumers integrate performance information for their evaluation of a close recommender in a 
way they do with distant recommenders is important, as it has immediate consequences for consumption decisions and ultimately for the adoption of products or services that are object of recommendations.

This dissertation is structured as follows: Chapter 1 provides an overview of established conceptualizations of recommendations and briefly reviews existing research on recommender evaluation. This literature reflects how recommendation performance affects the persuasiveness of recommenders and is a starting point for the question how evaluation of close others may differ from those of distant others. Following, we integrate both aspects, recommendation performance evaluation and relationship closeness, into our research framework. Chapter 2 and Chapter 3 form the empirical part of the dissertation. Here, we experimentally investigate the interplay of relationship closeness and recommendation performance on the intent to follow a recommender again (Paper 1) and how a close relationship to a recommender can ease negative consequences for firms after a service failure in first encounters (Paper 2). In both papers, we are especially interested in the mechanisms that drive the differential processing of recommendation performance information for close others versus distant others. To examine this question, we use mediation analysis. Chapter 4 (Paper 3) reflects on this statistical method and evaluates several approaches to establish mediation. Based on this conceptual reflection, we recommend how to conduct mediation analysis with the state-of-the-art methodology. In sum, Paper 3 underlines the rigor of the methods which have been used in the two empirical papers. The dissertation ends with general conclusions.

\subsection{Conceptualization of Recommendations}

To examine our research questions, it is first necessary to conceptualize our understanding of recommendations for the context of this dissertation. Recommendations among consumers are prevalently discussed in the realm of word-of-mouth (WOM) literature (Berger, 2014). They 


\section{Chapter 1}

can be regarded as a special form of WOM engagement as they are "person-to-person communication between a receiver and a communicator whom the receiver perceives as noncommercial, regarding a brand, a product, or a service" (Arndt, 1967, p. 3). According to Rosen and Olshavsky (1987), in a recommendation situation the recommender serves the recommendation receiver by simultaneously providing an alternative together with a positive evaluation for that alternative. This distinguishes recommendations from other WOM activities like product evaluations or gossiping about brands or companies as they can also entail negative connotations and thus may harm companies (Gershoff, Broniarczyk, \& West, 2001). Duhan, Johnson, Wilcox, and Harrell (1997) state that recommendations are a form of interpersonal communication among consumers concerning their personal experiences with a firm or a product. Thus, recommendations are commonly regarded as a way to help consumers decide for products or services (White, 2005; Yaniv, 2004). However, when consumers consult recommenders to support their decision making, they are also faced with the added responsibility of having to evaluate the recommender to ensure they only listen to suitable advisors (Gershoff et al., 2001). Prior research suggests that recommendation receivers use different types of information to assess recommenders (Feick \& Higie, 1992; White, 2005).

Across the diverse conceptualizations of recommendations, several characteristics of recommendations are consistently emphasized and thereby build the foundation for understanding the concept in this work, namely that recommendations are characterized by positive communication about products or services. They concern interpersonal communication among consumers that sets them apart from company or media communications. Recommendation situations encompass the person who provides the recommendation (recommender), the object of the recommendation (message), typically a service or a product, and the person who receives the recommendation (recommendation receiver). Finally, the 
recommendation receiver has to evaluate the recommender in order to increase the probability of suitable recommendation outcomes. This means, if consumers decide to rely on recommendations, they partly forward the responsibility of product choice to another person. However, they in return have to bear the responsibility of evaluating the recommender. Against this background, a considerable amount of research has examined which factors influence the evaluation of recommenders.

\subsection{Research on Recommender Evaluation}

Prior research has established that the decision to follow a recommendation is closely linked to a recommendation receiver's prediction that the person providing the recommendation is able to give valuable advice (Gershoff et al., 2001). Literature refers to this as recommender evaluation (Yaniv, 2004). An appropriate recommender evaluation would reliably reflect whether the recommendation provided by a specific recommender will match the preferences of the recommendation receiver. Two main approaches of recommender evaluation can be distinguished, evaluating recommenders based on recommendation performance and based on recommender characteristics.

Extant research suggests that recommendation performance is especially important for recommender selection (Gershoff et al., 2001; Gershoff et al., 2003; Harvey \& Fischer, 1997; Yaniv et al., 2011; Yaniv \& Kleinberger, 2000). It reflects the notion that performance in the past is predictive of performance in the present and thus individuals can learn from prior experience with a recommender. Indeed, recommendation performances seem to enable consumers to learn quickly about a recommender's appropriateness (Bonaccio \& Dalal, 2006). However, surprisingly scant research has explored how consumers actually integrate performance information in recommendation contexts. Only two studies explicitly address this question. Yaniv and Kleinberger (2000) investigated in a numerical guessing task how the 


\section{Chapter 1}

quality of a recommender's former advice influenced advice-taking intent in subsequent situations. Gershoff et al. (2001) examined the influence of recommendation performance when selecting between potential recommenders. While both works differ in their research context and presentation of information, they both show that a recommender is more influential if that person's recommendations were accurate before (Gershoff et al., 2001). Likewise, consumers are less likely to follow the recommender if past recommendations were inaccurate (Yaniv \& Kleinberger, 2000). Yaniv and Kleinberger (2000) also argue that negative performance information is particularly influential because it helps to identify inappropriate advisers. Other studies, though not explicitly examining recommendation performance, confirm that past opinion agreement with a recommender is an important cue for consumers' acceptance of current recommender advice (Gershoff et al., 2003; Yaniv et al., 2011). All these works manipulated prior performance of recommenders but did not provide additional information about recommender characteristics to the participants.

However, consumers often do not only consider recommendation performance. Instead, they also evaluate recommenders based on other characteristics that - in the perspective of the consumers - help to obtain good recommendation ability (White, 2005). Such inferences about recommender characteristics seems especially helpful when past performance information about a recommender is not available, for instance when a recommendation receiver has never followed a recommendation of this person. Prior research has shown that recommendation receivers value similar demographic profiles (Gino, Shang, \& Croson, 2009), similar goals (Twyman, Harvey, \& Harries, 2008), shared values (van Swol, 2011), and how well the recommender knows their preferences (Gershoff \& Johar, 2006). The underlying principle for relying on recommender characteristics is the belief that these characteristics can predict recommendation quality and that people who have these characteristics are good recommenders 
(Bonaccio \& Dalal, 2006). Relationship closeness is a source of belief that subsumes many beneficial characteristics and refers to the emotional association of relationship partners (Swann \& Gill, 1997). An example of people who have deep and involved relationships are close friends. More than strangers or acquaintances, friends care for each other and often know each other very well. Moreover, friends often have similar demographic profiles and share more similar attitudes and values than strangers (Lee et al., 2009; McPherson, Smith-Lovin, \& Cook, 2001). Friends can even become central to one's identity (Kenny \& Acitelli, 2001). Accordingly, people have high beliefs that friends know their personal preferences and needs especially well (Gershoff \& Johar, 2006) and thus are especially suitable recommenders (Eggleston et al., 2015).

\subsection{Focus of This Dissertation}

Although previous research has discussed various recommender characteristics that potentially affect people's willingness to accept recommendations, studies have not yet examined how these characteristics interact with the utilization of recommendation performance information. Specifically, recommender characteristics such as similarity or expertise widely appear in the word-of-mouth literature streams (Brown \& Reingen, 1987; Faraji-Rad, Samuelsen, \& Warlop, 2015; White, 2005; Yaniv, Choshen-Hillel, \& Milyavsky, 2011), but these streams have neglected the role of recommendation performance. Although Gershoff et al. (2001) acknowledged the parallel presence of performance information and recommender characteristics, research has yet to examine their interaction. Likewise, research has not yet paid attention to the question of how recommender characteristics may influence the use of performance information. This is surprising because, in many recommendation situations, consumers have both, recommendation performance and inferred recommender characteristics available (Feick \& Higie, 1992). 


\section{Chapter 1}

The two empirical papers of this dissertation examine how recommendation receivers deal with the situation when an interrelational recommender characteristic (i.e., relationship closeness) suggests favorable recommender evaluation, but at the same time an unsuitable recommendation performance suggests the opposite. While Paper 1 analyzes the behavioral consequences of bad recommendation performance on the intent to follow the same recommenders in subsequent recommendation situations, Paper 2 focuses on the consequences of relationship closeness to recommenders for companies that have caused a service failure in an initial encounter. The contribution of this dissertation is to integrate both aforementioned evaluation approaches and address the identified research gap by investigating whether, when and how performance of close (vs. distant) recommenders affects recommendation receivers' response differently. To our best knowledge our work is the first that focuses on the behavioral consequences in case close others have given bad advice.

\subsection{Research Outline}

The dissertation comprises three papers. In the first two papers we examine how recommendation receivers react to unfavorable outcomes that result from recommendations of close recommenders. First, we examine the joint effects of negative recommendation outcomes and relational closeness to a recommender on the intent to follow this person again (Paper 1). Second, we examine how recommendations from close others may also alter the reasoning about a service failure that has occurred after a recommendation. Specifically, we suggest that a close relationship to a recommender may influence causal reasoning and behavioral consequences after service failure in a beneficial way for this firm (Paper 2). Paper 3 is a methodological contribution that comprehensively examines mediation analysis, a group of methods that explore the causal mechanisms by which a predictor affects an outcome. This analysis is used in both empirical papers. Figure 1 shows the dissertation's framework. 


\section{Figure 1: Dissertation Framework.}

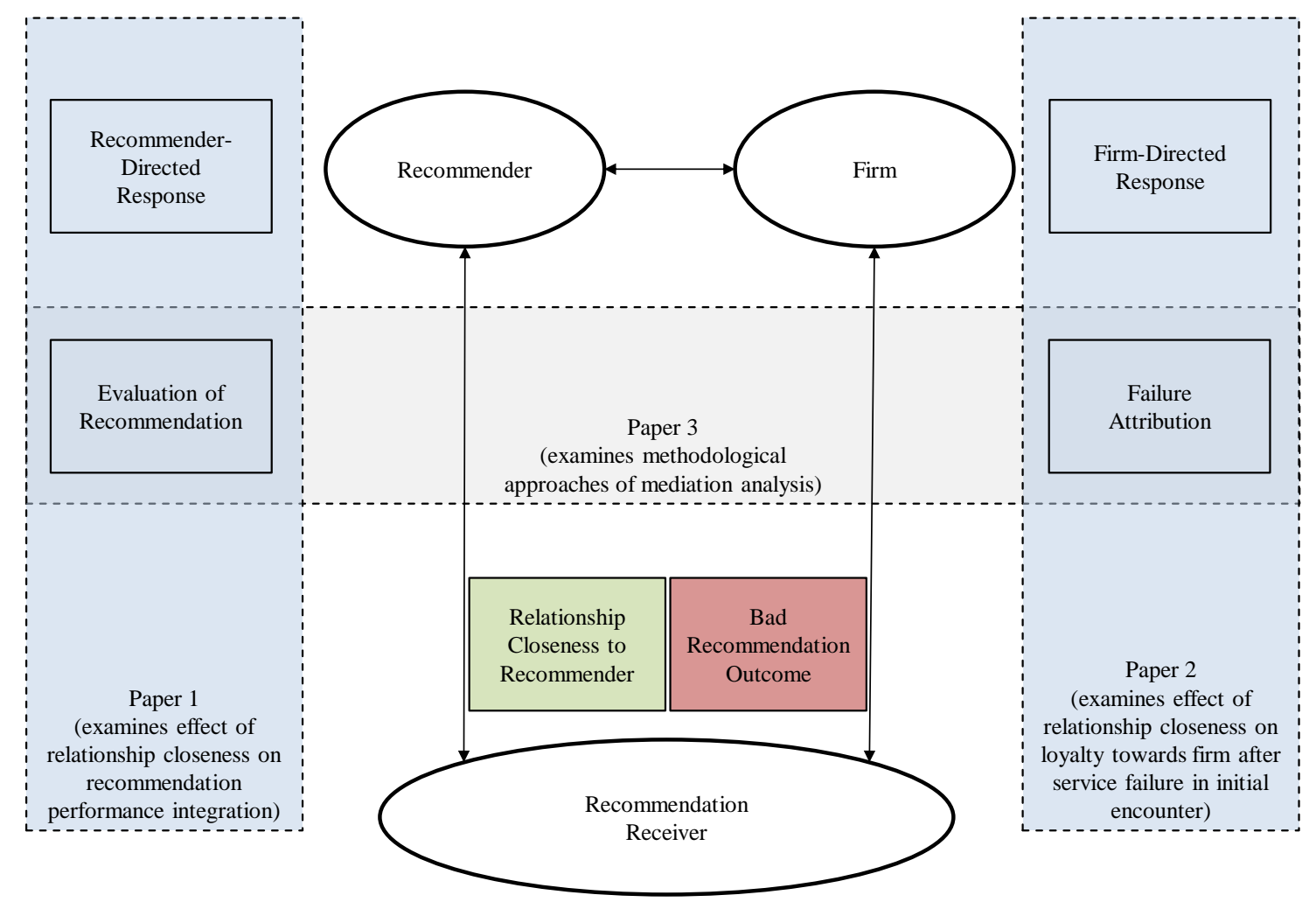

The framework points out the interrelations of recommendation receivers, recommenders, and firms as stakeholder in recommendation contexts and provides an overview about the specific approaches of all three papers. First, this framework illustrates the impact of relationship closeness on recommender evaluation and helps to understand potential effects of relationship closeness in recommender evaluation (e.g., sticking with an unsuitable recommender). Second, the framework points to consequences of relationship closeness on other stakeholders in the recommendation context. Here, our focus is on the effects that relationship closeness to a recommender can have for firms in case of service failure. Drawing on attribution theory we explain why recommendations of close others may help companies to get a second chance after a service failure in the initial encounter with a customer. This 


\section{Chapter 1}

perspective deepens the understanding of buffering negative response to service failure when encounters result from recommendations. Finally, we take a methodological perspective that supports the rigor of our statistical analysis and extends the understanding of mediation analysis. Table 1 summarizes the research goals, key findings, and key contributions of the papers. 


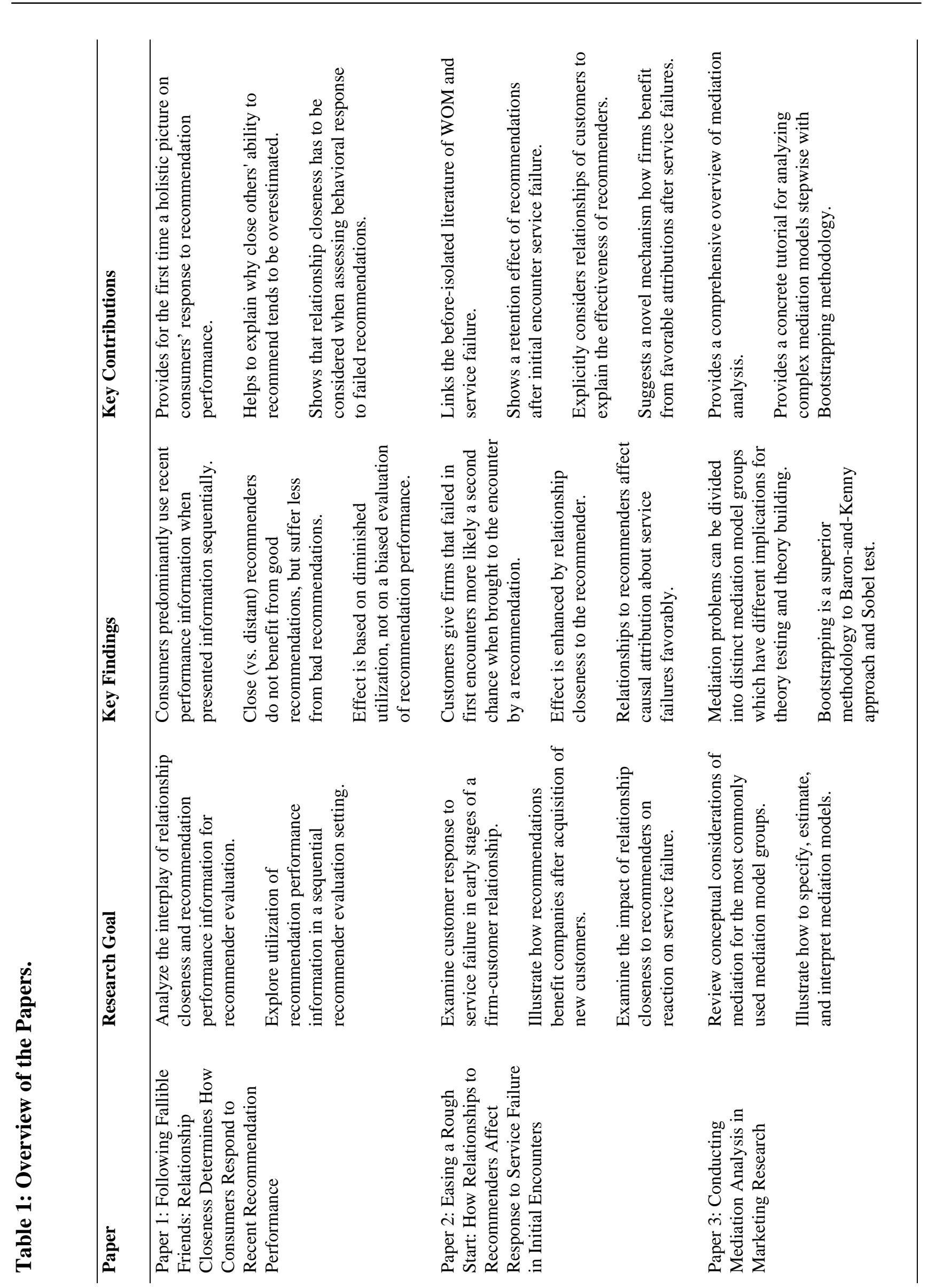




\section{Chapter 1}

Each of the paper faces different methodological challenges. The two empirical papers employ a portfolio of established analytical methods such as analysis of variance, logistic regression analysis, bootstrapped mediation (moderation) analysis, and methods for addressing selection, heterogeneity and endogeneity issues. Table 2 gives an overview of the data, sampling, research context, and methodology of the two empirical papers. In both papers, we conducted an exploratory pilot study which paved the way for the following studies. Furthermore, we used a scenario-based experimental approach to test our conceptual frameworks. This approach is common for measuring the effects of recommendation performance on consumer reasoning in recommendation research (e.g., Gershoff \& Johar, 2006; Gershoff, Mukherjee, \& Mukhopadhyay, 2003; Yaniv \& Kleinberger, 2000). With regard to research context, Paper 1 follows previous recommendation research focusing on domains which are characterized by preference heterogeneity like movies (e.g., Gershoff \& Johar, 2006). Other reasons for the choice of our contexts were the results of our pilot studies that suggested domains where recommendations among consumers are especially common. In Paper 2, we aligned our research contexts with prior research on service failure and created our scenarios accordingly. Specifically, we followed previous research that examined service failure in the domain of primary care physicians (Mittal, Huppertz, \& Khare, 2008) and in the restaurant domain (Cheng \& Lam, 2008; Mittal et al., 2008). Also, we manipulated service failures in a similar way to previous research (Bitner, Booms, \& Tetreault, 1990) to make our results comparable. A detailed description of our considerations is provided in the data and methodology sections of the respective papers. 

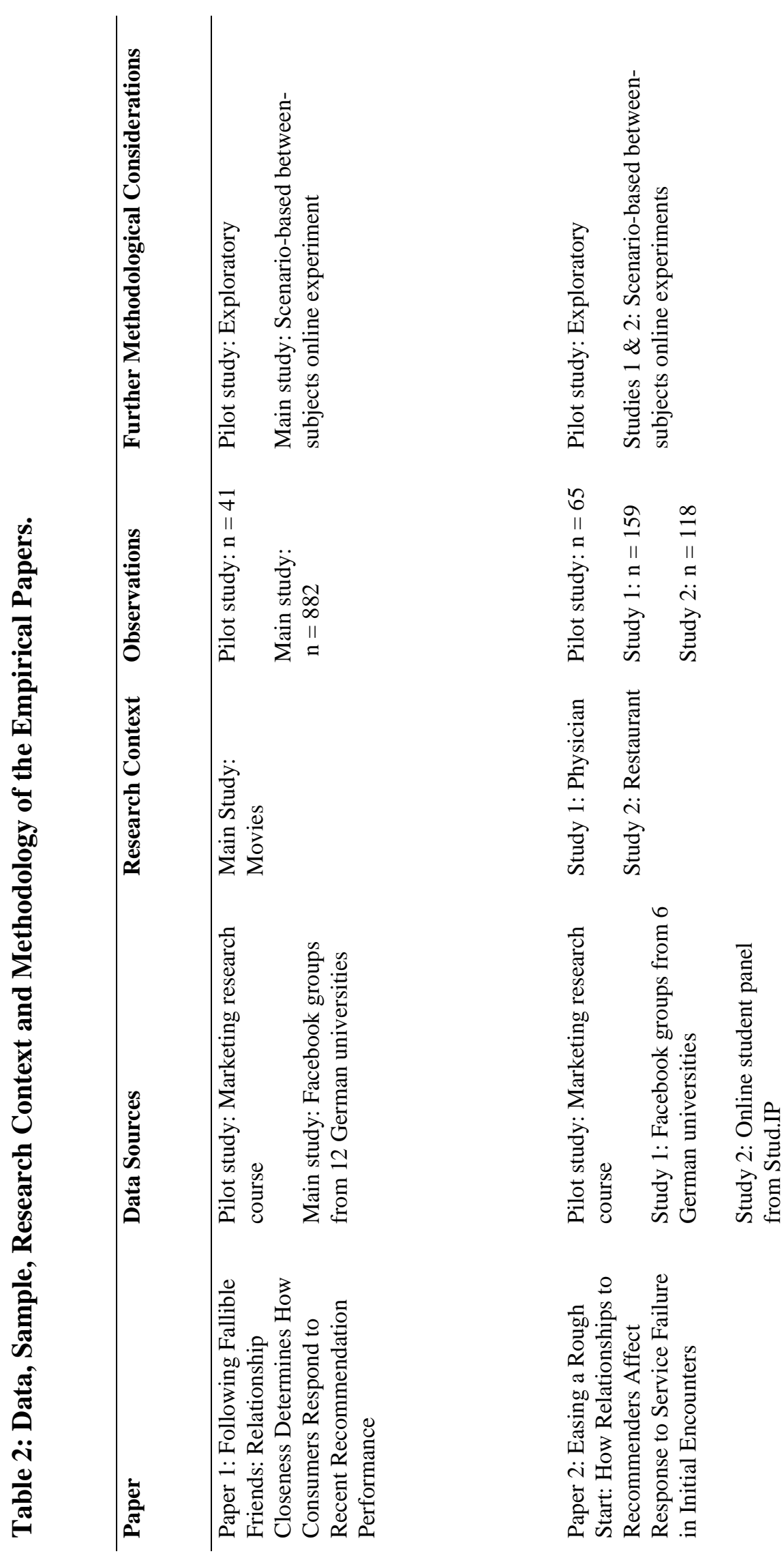


\section{Chapter 1}

Paper 3, as conceptual paper, is based on a systematic review of 65 methodological articles on mediation analysis from top-tier journals. Furthermore, a content analysis analyzing in 752 articles in top-5 marketing journals illustrates the rapidly gained importance of mediation analysis in the last 40 years has and shows which approaches are applied by marketing researchers. In addition, it provides a tutorial and syntax for common models in regressionbased mediation analysis.

The next chapter provides the abstracts of the papers.

\subsection{Abstracts}

\subsubsection{Paper 1}

Consumers often decide whether they follow a recommender based on his or her prior performance. In this article, we argue that as consumers integrate information sequentially they especially respond to recent performance of a recommender - regardless of the total number of prior recommendations. Drawing on the notion that close relationships activate social beliefs, we further argue that consumers utilize recent performance differently when they have a close relationship to a recommender. Specifically, we show that consumers respond less to a failed recommendation from a friend than to one of an acquaintance. Interestingly, the response to recent good recommendation performance is similar among friends and acquaintances. As a consequence, consumers are more susceptible to stick with unsuitable recommenders when these are friends. In sum, we provide a recommender evaluation framework that explicitly considers sequential performance processing as well as the impact of relationship closeness between recommenders and recommendation receivers. 


\subsubsection{Paper 2}

First encounters with service providers are crucial for the trajectory of firm-customer relationships. Therefore, it may be critical when a service fails during the initial touchpoint with a customer. The purpose of this paper is to examine how recommendations from other consumers affect customer response to service failure in the first encounter with a service provider. Two scenario-based online experiments show that recommendations from other consumers enhance customer loyalty in comparison to traditional marketing after initial encounter service failure. Furthermore, we find that especially the recommendations from close others have a positive impact on customer retention. By parallel mediation analysis we examine the underlying psychological mechanisms that drive the beneficial effect of close relationships to recommenders. We show that customer retention effect of recommendations of close others stems from a shift in failure attribution of new customers. Our suggested conceptual framework helps to examine the role of relationships to recommenders in an initial encounter service failure setting. Thus, this paper provides insights into how recommendations can help to retain service relationships that went wrong after acquisition. This paper is one of the first to address a mechanism that links recommendations of close recommenders to service failure literature. Moreover, this paper provides guidance for managers to re-assess the value of other consumers for strategies to retain new customers after service failure.

\subsubsection{Paper 3}

Marketing researchers frequently conduct mediation analysis to enrich their understanding of a focal causal relationship by examining its underlying mechanism. The main purpose of this review is to provide an overview of what mediation analysis means, which approaches exist to establish mediation, and how to conduct mediation analysis with the state-of-the-art methodology. In the first part of the paper we review conceptual considerations of mediation for the 


\section{Chapter 1}

most commonly used mediation model groups. We further discuss the suitability of different mediation analysis approaches, focusing on the bootstrapping approach. The second part of the paper is organized as a tutorial. Based on an example from the marketing field, we illustrate how to specify, estimate, and interpret mediation models with a tool for SPSS and SAS called PROCESS (Hayes 2017). We recommend a hierarchical procedure in which simple mediation models are examined first, followed by more complex models. 


\section{Following Fallible Friends: Relationship Closeness Determines How Consumers Respond to Recent Recommendation Performance (Paper 1)}

(With Steffen Jahn and Yasemin Boztuğ $)^{1}$

Earlier versions of this paper have been under review in Marketing Letters, 2020 and in Journal of Consumer Psychology, 2019.

Prior versions of the paper were presented as:

When Friends Give Bad Advice: How Relationship closeness Impacts Evaluation of Recommendations and Willingness to Follow Again. EMAC Conference, Glasgow, UK, 2018.

The Impact of Relational Closeness on Advice Taking After Prior Inaccurate Recommendations. EMAC Doctoral Colloquium, Groningen, Netherlands. 2017.

Keywords: Recommendations; Agent Evaluation; Motivated Reasoning; Relationship Closeness; Word-of-Mouth; Recency Bias

\footnotetext{
${ }^{1}$ This paper was created in cooperation with the listed co-authors. I was responsible for the literature review, the theoretical framework, the hypotheses development, the data management, the methodology, and the empirical analysis. My co-responsibilities comprised the positioning, the contribution statement, the conceptual framework, and the implication section.
} 


\section{Chapter 2}

\subsection{Introduction}

Imagine you received a bad movie recommendation from a friend. Would you follow another movie recommendation from this friend? Research on recommender evaluation suggests that this decision would be influenced by the friend's prior performance in recommending movies (Gershoff et al., 2003). Specifically, consumers are thought to weigh in all good and bad outcomes of former movie recommendations to assess the probability of a successful movie recommendation in the future (Gershoff et al., 2001). If, for example, the friend has previously provided 30 out of 40 good movie recommendations, one recent bad recommendation would not ruin the overall favorable success rate. If, however, the friend would have made only 3 out of 4 good movie recommendations before, one additional bad recommendation would substantially lower his or her success rate, implying that one would less likely follow the next recommendation.

Past research has established that consumers use information on recommendation performance when a summary is provided that lists all past successes and failures of a recommender (e.g., Gershoff et al., 2001; Gershoff et al., 2003, 2007). However, it remains unclear how consumers apply performance information when they deal with a recommender sequentially. In this article, we argue that consumers might not keep an exact tally of past recommendation performance and instead might react more strongly to recent events. Furthermore, while many authors (e.g., Berger, 2014; Brown \& Reingen, 1987; Duhan et al., 1997) acknowledge that recommendations are often given by persons who are close, extant research largely ignores the consequences that relationship closeness has for recommender evaluation and how recommendation performance from close others is processed. Provided that close relationships activate social beliefs which influence downstream behaviors (Cavanaugh, 2016), we suggest that response to a failed recommendation from a friend might differ from 
one of a less close recommender. We develop a framework that explicitly considers sequential performance processing as well as the impact of relationship closeness on utilization of performance information. The framework draws from literature on information integrationsuggesting that whenever people process information sequentially, weighting of information may deviate from having information available all at once (Hogarth \& Einhorn, 1992) —and motivated reasoning - suggesting that belief-inconsistent information may be discounted (Klein \& Kunda, 1992).

By integrating sequential information processing and motivated reasoning frameworks in the context of recommender evaluation, we contribute to extant literature in several ways. First, while past research has explored how consumers use recommendation performance when they are presented with all information at once (e.g., Gershoff et al., 2001; Yaniv, Choshen-Hillel, \& Milyavsky, 2011), to the best of our knowledge, no work to date has explored how sequential information presentation affects recommender evaluation. This work helps to understand the interplay of recent and less recent performance information that drive recommender evaluation in sequential recommendation contexts.

Second, our research extends what is known about consumers' use of recommendation performance information in the context of recommendations from persons with close relationships. Our research design jointly considers recommenders' past performance and their relational closeness and makes it possible to explain whether potentially different responses to failed advice of friends stem from their relational involvement or from higher exposure to closer recommenders (i.e., a higher number of past recommendations). Specifically, by examining recent performance information that is not consistent to beliefs about close others, we find an 


\section{Chapter 2}

asymmetric effect, in that relationship closeness diminishes utilization of recent poorperformance information but does not affect good-performance information.

\subsection{Theoretical Background}

We build our conceptualization on research highlighting the importance of prior performance for recommender evaluation (Gershoff et al., 2001; Gershoff et al., 2003; Harvey \& Fischer, 1997). In empirical studies, recommendation performance is typically operationalized by the total number of good and bad performances a recommender has given. For example, Gershoff et al. (2001) provided a table showing all past successes and failures of potential recommenders at once.

A shortcoming of presenting performance information simultaneously is that in real life, consumers rarely have a full account of a recommender's performance, i.e., a record of all successful and failed recommendations. Instead, it is more natural for individuals to process information sequentially (Anderson, 1981). Specifically, we know from impression formation literature that consumers tend to process pieces of performance information once at a time (Denrell, 2005). Thus, it is conceivable that the evaluation of recommenders also occurs sequentially. It is important to note that processing information sequentially may change how consumers weigh information (Hogarth \& Einhorn, 1992). One effect that is especially likely to occur is focusing on recent information at the expense of less recent information as the former can be readily recalled (Tubbs, Gaeth, Levin, \& van Osdol, 1993).

In line with our theorizing, Yaniv and Kleinberger (2000) found that the quality of a person's recent advice in a sequential numerical estimation task influenced how much participants departed from their own estimate in favor of the advisor's estimate in a subsequent task. This influence of advice quality seemed to fade away after several subsequent sessions. 
In summary, we argue that simultaneous and sequential performance may lead to substantial differences in recommender evaluation. While presenting performance simultaneously lends importance to the total number of recommendations given by the recommender, the total number of recommendations becomes less relevant in sequential information presentation. This implies that response to a recent negative recommendation will not depend on how many recommendations a person has given previously. This leads to our first hypothesis:

H1: A recommender's recent performance will influence the intention to follow this recommender again regardless of the total number of recommendations the recommender has previously provided.

It is important to note that our premise is not to state that consumers do not utilize at all recommendation performance information that dates back long ago. Instead, we suggest that the number of recommendations a recommender has given plays only a subordinate role compared to the weight of recent information. This suggestion at least questions the external validity of prior studies that presented performance information simultaneously (e.g., Gershoff et al., 2001; Gershoff et al., 2003).

Next, we examine the role of relationship closeness in recommendation settings. Previous work on recommender evaluation has used scenarios where participants did not actually know their recommenders (e.g., Gershoff et al., 2001; Yaniv et al., 2011). Recommenders were either introduced as anonymous participants from prior sessions or as hypothetical recommenders and overviews of their recommendation performance were provided. Performance information had strong effects. However, consumers did not value all performance information equally. For example, consumers consider information about bad 


\section{Chapter 2}

performances especially meaningful and thus utilize negative performance information more than positive information (Yaniv \& Kleinberger, 2000). In contrast, recommendation performance in favor of a product seems to be more important than against it (Gershoff et al., 2003, 2007). Notably, these effects are based on characteristics of the recommendation and not of the recommender. Provided that in many real-life recommendation settings consumers have a relationship with their recommenders, consideration of this social information seems warranted.

Relationship closeness reflects the degree of emotional attachment between individuals (Swann \& Gill, 1997). Prior research has shown that close others influence consumer behavior in many domains (Cavanaugh, 2016). Consumers have strong beliefs about the persons they feel closest to, for example, that these persons know their preferences especially well (Kenny \& Acitelli, 2001). A relevant example of a close relationship is friendship. Friends frequently provide recommendations and are often solicited for advice (Brown \& Reingen, 1987). Moreover, friends care for each other and value their relationship. Prior research also suggests that consumers strongly believe that friends will make superior personal recommendations (Eggleston, Wilson, Lee, \& Gilbert, 2015).

However, these beliefs are not always correct. Gershoff and Johar (2006) found that individuals not only over-estimated how well friends knew their preferences, they also adjusted their estimate to a higher extent when friends knew more than expected than when less than expected. This positivity bias was not found for acquaintances. The authors explained their results by individuals' motivation to defend beliefs that are based on relationship closeness.

We draw on this motivational account and transfer it from attitudinal calibration of friend's knowledge based on simultaneously presented feedback to a sequential 
recommendation situation including behavioral intent toward a recommender. Because belief in friends as recommenders reassures individuals about the closeness of their relationship (Kenny \& Acitelli, 2001), we argue that consumers may be motivated to downplay the diagnostic value of a recent performance if it is incompatible to their belief. Specifically, in the case of friends giving bad recommendations, the utilization of information on negative performance may be reduced.

The underlying rationale stems from motivated reasoning research showing that retaining beliefs is an important goal for individuals (Kruglanski \& Freund, 1983). Beliefretention goals may lead to selective processing of information that is consistent with existing beliefs. In other words, people use those pieces of information they consider most likely to yield a preferred judgment (Klein \& Kunda, 1992). For example, individuals utilize information that supports their desired impression of a target person and inhibit information that interferes with it (Kunda \& Thagard, 1996).

In our context, we expect the goal of following only suitable recommenders is sacrificed for the goal of maintaining the belief in friends as recommenders. This effect should be strongest when performance information is negative and thus directly opposes beliefs underlying the relationship. Thus:

H2: Relationship closeness decreases utilization of recent performance information, especially of negative recent performance information.

\subsection{Pilot Study}

We conducted a pilot study to explore in which domains personal recommendations from others frequently occur and how well people can recall performance information about recommenders. 


\section{Chapter 2}

Forty-one business students (63\% female; average age: 23.4 years) participated as part of a marketing course.

First, we asked the participants to write down all categories in which personal recommendations are common to them and mark those they considered most important. Each participant listed between one and seven categories which we grouped into 15 broader domains. The most frequently stated domains were movies/TV shows (mentioned by $73 \%$ of participants), restaurants (19\%) and food (13\%). Movies/TV shows was also the domain that the highest share of participants considered as especially important for personal recommendations $(39 \%)$.

Second, we explored how the participants evaluated a specific recommender. Therefore, we asked the participants to think of a concrete person who regularly gives them personal recommendations in their most important domain. Next, we asked (1) how they evaluated the most recent recommendation of that person in the chosen domain (three answer options: good, not so good, do not know), (2) how many recommendations that person had given in total in the domain and how many of those turned out (3) good and (4) bad. Finally and most important, the participants were asked whether they felt able to answer each of these four questions reliably (answer options: yes, no). Thereby, we measured the participants' perceived ability to evaluate a recommender with regard to the most recent performance, the total number of recommendations and the total number of good as well as bad performances.

Most of the participants stated that their chosen recommender gave a good recommendation recently $(90 \%)$. The median number of recommendations they received from that person was 7. To calculate the success rate of the recommender, we divided the stated number of good performances by the stated number of bad performances. As expected the 
median ratio of good and bad performances was high (4:1), but only $29 \%$ of the participants reported that they never received a bad recommendation from that person. Finally, we analyzed how confident participants were in reliably recalling performance information. Notably, most participants stated that they felt able to recall the most recent performance well (76\%) and also how many recommendations the recommender had given in total $(63 \%)$. However, only few participants were confident in assessing the total number of good or bad performances well (34\% and $29 \%$, respectively).

The pilot study suggests that consumers have a good sense of recent recommendation performances, but they appear to have difficulty in assessing the overall performance of a recommender, i.e., how many good and bad recommendations this person has given in total. These results provide first support for our reasoning. In the main study we investigate the impact of recent recommendation performance on the decision to follow a recommender again. Further, the study tests whether a close relationship to the recommender diminishes the extent to which recent recommendation performance is utilized.

\subsection{Main Study: Movie Recommendation}

\subsubsection{Participants and Design}

Eight hundred and eighty-two undergraduate and graduate students (71\% female; average age: 24.3 years) participated in the experiment in exchange for a chance to win an Amazon.com voucher. We randomly assigned the participants to one of four conditions in a 2 (recent recommendation performance: good vs. bad) $\times 2$ (relationship closeness: acquaintance vs. friend) between-subjects design. As the pilot study suggested that movies are a common domain for recommendations, we chose this context for the recommendation setting. 


\section{Chapter 2}

\subsubsection{Procedure}

Participants read a scenario ${ }^{2}$ where they received a movie recommendation from a person they know. To manipulate relationship closeness with this recommender, we asked the participants to think of a close friend (acquaintance) of theirs who would act as the recommender and to type this person's name. The questionnaire referred to the recommender's name several times to make the mental representation of the real person in the hypothetical context more vivid.

Participants were asked to imagine that they met their friend (acquaintance) by chance and told them about their plans to go to the cinema, without yet having a movie in mind. The person then provided a movie recommendation. After the participants stated how likely they would follow this initial recommendation (measured on a 7-point scale, $1=$ definitely not, $7=$ definitely), they were told to imagine they had seen the recommended movie thereafter and were provided with a general (not movie- or genre-specific) five-sentence vignette that described the movie. Thereby, we manipulated either a good or bad recent recommendation performance. The vignette, chosen from a pretest (pretest vignette evaluation: $M_{\text {good }}=6.11 \mathrm{vs}$. $\left.M_{b a d}=2.17 ; t(52)=18.05, p<.001\right)$, contained information about several movie dimensions, either phrased positive or negative. As our main target variable, the participants stated how likely they would follow again if their friend (acquaintance) provided another movie recommendation next time they were going to the cinema (measured like initial intent to follow). We also asked the participants how many movie recommendations the recommender had given the participant in the past to test if recent performance information utilization was dependent on the total number of recommendations.

\footnotetext{
${ }^{2}$ Stimuli and measures of this study are provided in Appendix 1A.
} 
As a first manipulation check, we measured the participants' evaluation of the recent recommendation performance with three items: matching taste, quality of the movie, appropriateness of the recommendation (measured on 7-point scales). Our second manipulation check measured relationship closeness to the recommender by an adapted scale from Swann and Gill (1997): the participants stated the time spent doing things with the recommender, conversing with the recommender, sharing problems and worries with the recommender, and their liking of the recommender (measured on 7-point scales). First checks confirmed the internal consistency of our measures of recent performance evaluation $(\alpha=.95)$ as well as relationship closeness $(\alpha=.90)$ and indicated that our manipulations worked as intended: the participants in the good performance condition rated the recent recommendation performance more favorably than those in the bad performance condition $\left(M_{\text {good }}=5.52\right.$ vs. $M_{b a d}=2.79$; $t(880)=39.67, p<.001)$. Furthermore, relationship closeness was higher in the friend condition than in the acquaintance condition $\left(M_{\text {friend }}=5.55\right.$ vs. $M_{\text {acquaintance }}=3.18 ; t(880)=$ $37.81, p<.001)$.

\subsubsection{Results}

Intent to Follow Again. We analyzed the data by a two-way analysis of covariance (ANCOVA) using our manipulations of recent performance and relationship closeness as binary independent variables to explain intent to follow the recommender again, controlling for the total number of prior movie recommendations from the recommender.

As expected, the intent to follow again was higher for recommenders who were friends compared to those that were acquaintances $\left(M_{\text {friend }}=5.15\right.$ vs. $M_{\text {acquaintance }}=4.10$; $F(1,824)=16.58, \quad p<.001)$. Furthermore, participants were more likely to follow recommenders after a good recent recommendation $(M=5.54)$ than after a bad one $(M=3.76$; 


\section{Chapter 2}

$F(1,824)=43.48, p<.001)$. However, the number of prior movie recommendations from the recommender did not affect the intent to follow again $(p>.1)$.

Hypothesis 1 stated that a recommender's recent performance will influence the intention to follow again regardless of the total number of recommendations the recommender has given previously. In line with this hypothesis, moderated regression results indicate a nonsignificant interaction between recent recommendation performance and the number of prior movie recommendations from the recommender $(p>.4)$. Thus, the participants utilized recent recommendation performance independent of prior experience with the recommender. Specifically, poor (vs. good) recent recommendation performance had a significant negative effect across low $(b=-2.57, p<.001)$ and high $(b=-2.10, p<.001)$ levels of number of prior recommendations from that person.

Next, we analyzed the impact of relationship closeness on the utilization of recent recommendation performance. Hypothesis 2 stated that relationship closeness attenuates utilization of recent performance information, especially of negative recent performance information. As predicted, we found a significant interaction of recent recommendation performance and relationship closeness $(F(1,824)=5.67, p=.023)$. As Figure 2 shows, when the recommender was an acquaintance, recent recommendation performance had a high impact on the intent to follow. Thus, it acted as a strong signal for participants that assessed a recommendation from an acquaintance $\left(M_{\text {good }}=5.30\right.$ vs. $M_{b a d}=2.79 ; F(1,877)=357.59$, $p<.001)$. Although recent performance also impacted the intent to follow a friend's recommendation $\left(M_{\text {good }}=5.75\right.$ vs. $\left.M_{b a d}=4.55 ; F(1,877)=99.08, p<.001\right)$, its effect was less substantial. In terms of percentage change, a recent bad performance (vs. a good one) of an acquaintance resulted in an almost halved intent to follow (-47\%), whereas loss of influence due to recent poor performance of a friend was substantially lower $(-21 \%)$. 


\section{Figure 2: Effect of Recent Recommendation Performance on Intent to Follow Friends} and Acquaintances Again.

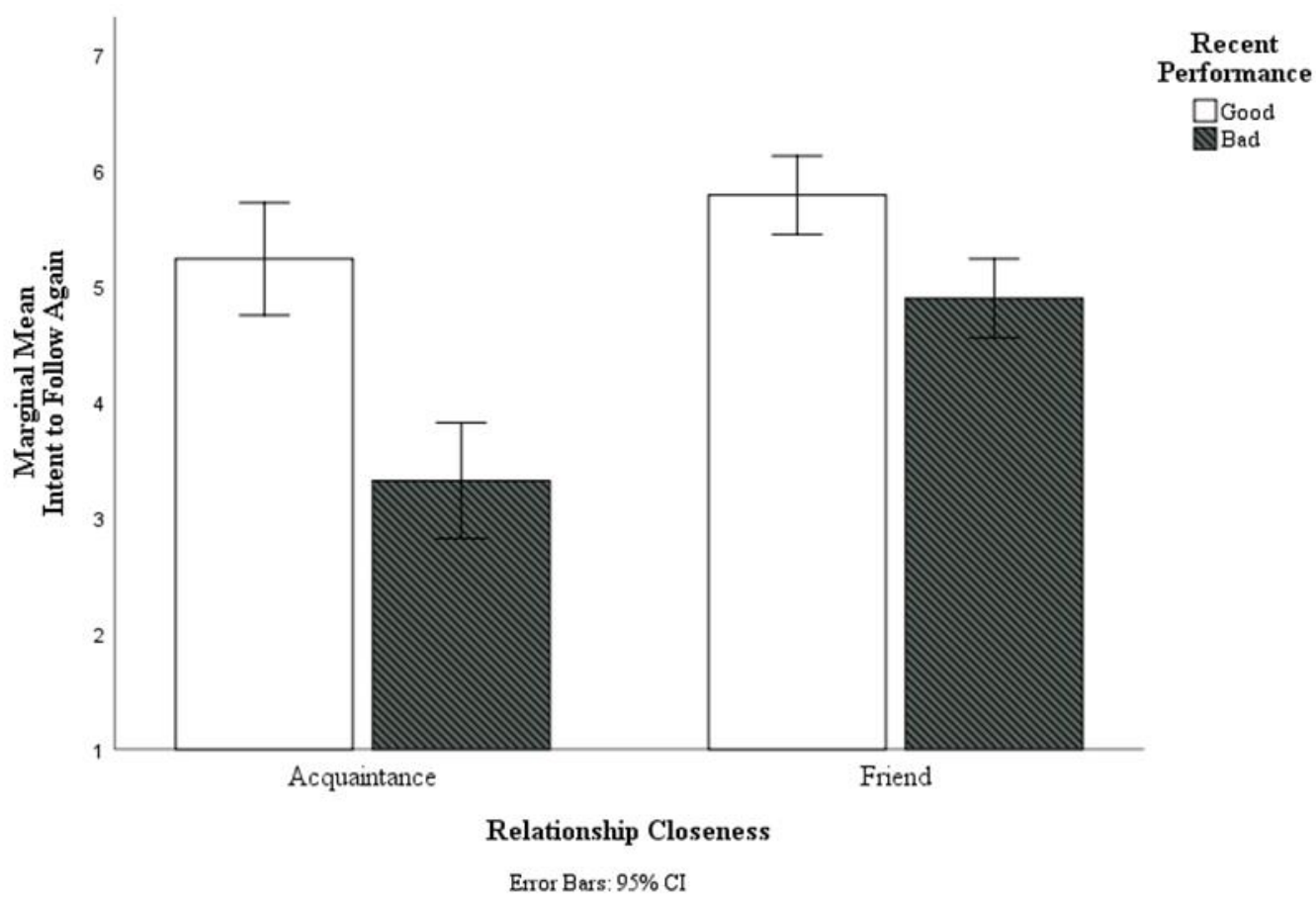

Comparing both relationship closeness groups for good recent performance showed only a small difference regarding the intent to follow the recommender again (mean group difference $=.45$ ). This means acquaintances ended up only slightly less influential than friends after providing a good recommendation. However, after providing a bad recommendation, the mean difference between the intent to follow a friend and an acquaintance was four times as high (mean group difference $=1.76, p<.05)$.

Initial Intent to Follow. We showed that consumers responded less to a recent failed recommendation from a friend than to one of an acquaintance, whereas the response to a recent good recommendation was similar among friends and acquaintances. While this result is in line with our theorizing, one might argue that the response to recommendation performance may be partly driven by the initial level of intent to follow a recommender. For example, recommenders that start from low levels of intent to follow might gain more influence after a recent good 


\section{Chapter 2}

recommendation than those that start from high levels, simply because they offer more potential to adjust upwards. As friends likely have a high starting level, they might potentially benefit less from recent good recommendations. This ceiling effect for friends might have biased our results.

In contrast, a high starting level also means more potential to adjust downwards after recent bad recommendations. If this effect might have impacted our results, it makes it more likely that friends respond more to recent bad recommendations. Thus, our experiment can be regarded conservative for diminished response of friends to bad performance. To rule out these potentially biasing influences, we compared the response of both relationship closeness groups on identical levels of initial intent to follow. Therefore, we split the data by manipulated recent performance and the levels of initial intent to follow (see Figure 3).

In this analysis we did not consider those levels with only few participants in either of the two relationship closeness groups (level 1 or 2 , friends: $n=7$; level 7 , acquaintances: $n=$ 15). Pair-wise comparisons via t-tests in the remaining four levels showed that after recent bad recommendations consumers update their intent to follow in a subsequent recommendation less if these recommendations came from friends, compared to acquaintances (Figure 3, left panel). However, there were no differences between friends and acquaintances in utilizing recent positive performance information (except the very small one at level 5, Figure 3, right panel). These results demonstrate the robustness of our findings.

Performance Evaluation. So far, we have argued that the response to bad recommendation performance of friends is based on a motivation to utilize belief-inconsistent performance information less. However, research has shown that the motivation to yield a desired conclusion 


\section{Figure 3: Effect of Recent Recommendation Performance by Initial Levels of Intent to} Follow.

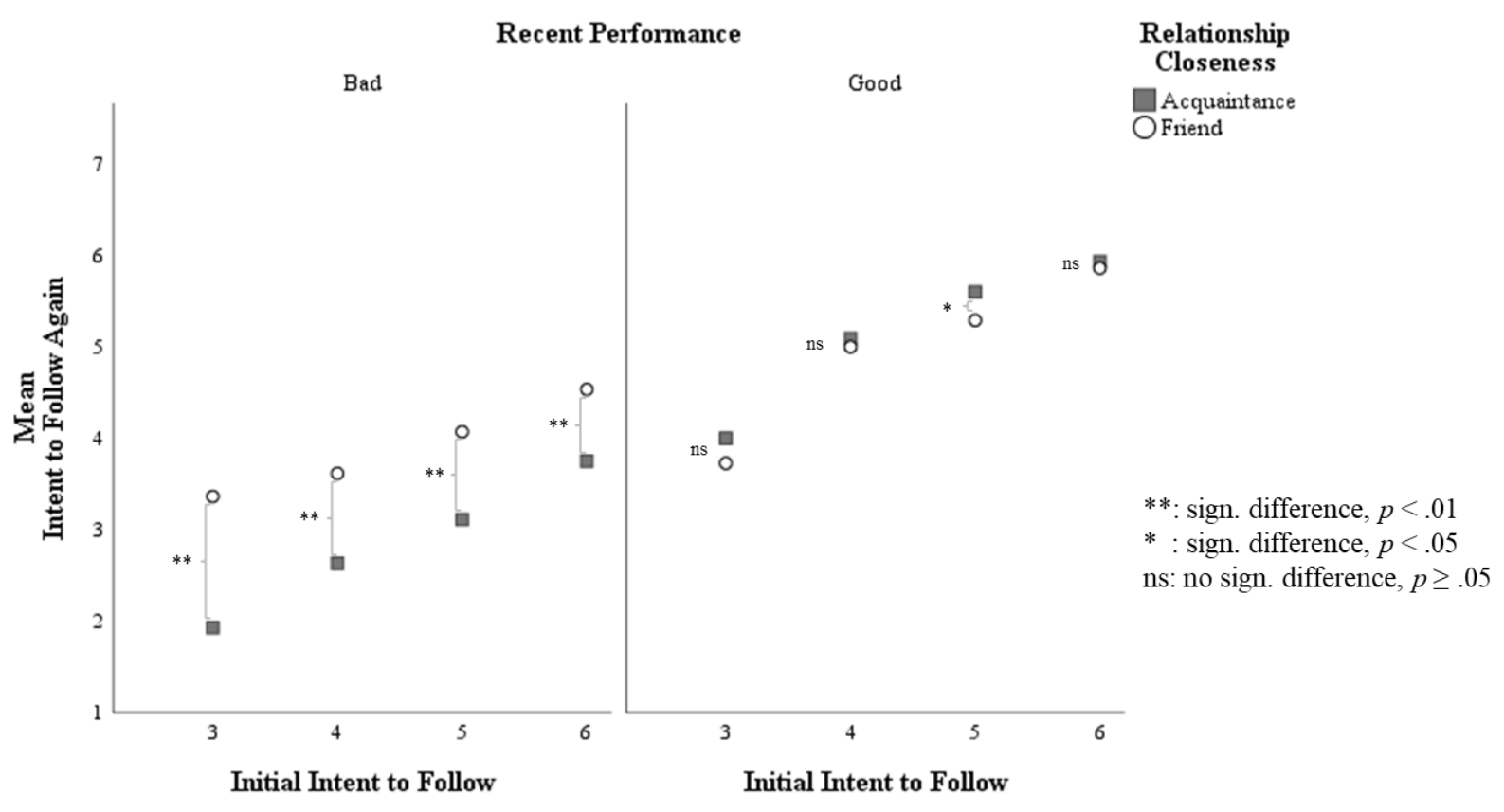

may also be achieved by evaluating the belief-inconsistent information in a biased, more favorable way. This would mean that participants potentially responded less to the bad performance of friends because they actually evaluated recommendation outcomes more benevolently when they came from friends than when they came from acquaintances. To clarify whether the differential response to bad recent performance information can be explained by a bias in utilization of information or a bias in evaluation of this information, we added the measured evaluation of the recent recommendation as a mediator to our model. We kept the binary manipulation of recent performance as independent variable and intent to follow again as dependent variable, controlling for the total number of prior movie recommendations from the recommender. Importantly, we allowed relationship closeness to act as a moderator potentially influencing the path from recent performance to evaluation $\left(1^{\text {st }}\right.$ leg moderated 


\section{Chapter 2}

mediation, Hayes, 2018) as well as the path from evaluation to intent to follow again $\left(2^{\text {nd }}\right.$ leg moderated mediation, Hayes, 2018). While the binary manipulation of recent recommendation performance heavily influenced the evaluation of the performance $(p<.001)$, relationship closeness did not impact the way participants evaluated this performance (the main effect of relationship closeness and the interaction effect of relationship closeness and recent performance were non-significant: $p s>.17)$. This result indicates that the differential response to negative recommendation performance of friends was not based on distorted evaluation of the recommendation performance. Instead, relationship closeness affected the way the evaluation of the performance was utilized $(b=-.35, p<.001)$. In other words, participants used information about recent recommendation performance of recommending friends less in their decision to follow that person's advice again. At the same time, they were fully aware of the quality of this performance and did not interpret it more favorably. Hayes's (2018) PROCESS macro (model 58, 5000 bootstraps) supported moderated mediation. In line with our previous findings, in the friend condition the indirect effect of recommendation performance on the intent to follow again was significantly lower than that in the acquaintance condition (index of moderated mediation: $1.09 ; 95 \%$ confidence interval [CI]: .80 to 1.40 ). In sum, we regard this as evidence that the asymmetric effect of relationship closeness on performance utilization is not produced by differences in evaluation of recommendation performance.

\subsection{General Discussion}

\subsubsection{Theoretical Contributions}

This research examined how consumers combine recent performances and relational bonds to evaluate recommenders, especially how they respond to bad recommendations from friends. This is relevant, as friends are frequent and impactful recommenders, but their ability to recommend tends to be overstated (Gershoff \& Johar, 2006). 
Our research extends what is known about the role of recommendation performance in several ways: First, we investigate a new setting. While research has established that consumers consider performance information when the advice relates to objective guessing tasks (Yaniv \& Kleinberger, 2000) or when selecting between two recommenders (Gershoff et al., 2001), we explore a sequential setting in which individuals need to decide whether to follow personal recommendations of a single recommender or not. We show that the impact of recent information in a sequential recommendation setting is not dependent on the number of prior recommendations from that person. Thus, the impact of recent information does not diminish with longer recommendation history. This result differs considerably from prior work that presented recommendation performance information simultaneously (Gershoff et al., 2001; Gershoff et al., 2003).

Our results also show that consumers are likely to stick with unsuitable recommenders when these are friends. Prior research has largely neglected such an interference of performance and relational cues and instead has contended that performance information works in parallel to other cues (Gershoff et al., 2001). We demonstrate that individuals use certain performance information less when recommenders are friends rather than acquaintances. While the response to recent good recommendation performance was similar among friends and acquaintances, we find that individuals considered bad performance information less when recommenders were friends. Therefore, our findings qualify prior research by considering the moderating effect of relationship closeness.

In our work, we took a motivational perspective to better understand when relationships influence the effect of performance information (and when they do not). Combining our results with work on beliefs about friends as recommenders (Eggleston et al., 2015) shows that 


\section{Chapter 2}

relationship closeness diminishes the consequences of performance information only when this information is not belief-consistent.

We also show that when beliefs are not threatened (e.g., reaction to good recommendations of friends), relationship closeness is not important. This finding suggests that consumers do not always favor friends as recommenders. Consequently, we noted asymmetry in the effect of relationship closeness on the utilization of performance information. Thus, our results also add to the understanding of how consumer information processing can lead to inferior recommender selection (Gershoff et al., 2001).

\subsubsection{Public Policy and Managerial Implications}

Our research has direct implications for consumer welfare. Consumers appear vulnerable to repeatedly making unfavorable product decisions by relying on friends who either misjudge or ignore their personal preferences. The pattern of our results suggests that consumers downplay the diagnosticity of recent bad performances of friends to maintain beneficial beliefs about friends. One way to mitigate the problem is to educate consumers that friends may not always know them the best. Consumers could learn to contemplate the prior recommendation performance of friends to counteract the recency bias and the power of relationship closeness in their decision making.

In addition, our findings may also help companies. Company representatives often act as recommenders for customers. For example, salespeople advise clients on a regular basis. Our research suggests that deep relationships with clients can buffer response to recent advice that was suboptimal and may give salespeople a second chance to advise. Companies that have built close relationships with customers could even consider recommending more controversial options without the risk of instantly losing their influence. In contrast with "sure shots," such 
riskier recommendations often bear the potential to be an especially good fit or exceptional experience for customers. Also, our finding that a recent successful recommendation substantially enhances recommender influence when relationships are not close offers managerial implications. For example, companies could utilize an initial recommendation in a small matter with a new client to raise the acceptance rate for a subsequent focal recommendation. Such a sequential persuasive strategy would be similar to a foot-in-the-door technique (Scott, 1977).

Companies that use consumer recommendations as leverage can also learn from this research. We show that targeting friends for seeding strategies is reasonable as they are likely to be influential even when they did not give good advice previously. Another result of our research is that recommenders who have performed well recently are nearly as influential as close recommenders. These recommenders have not yet been targeted by seeding campaigns but offer high potential.

\subsubsection{Future Research Directions}

Further research could complement our findings in several ways. First, the sequential single recommender perspective we investigated deserves more attention as it occurs in many lowstakes product decisions. Often consumers may not make the effort or be able to recall all prior performances of a recommender. Also, they may not ask for a second opinion of another recommender or invest extra effort in searching for further product information when the significance of a product decision is low. In such situations, a recommendation may immediately influence product decisions. One promising avenue for further research is to qualify established findings in recommender evaluation by taking a sequential perspective that highlights the order of information integration. For example, consumers seem to pay special 


\section{Chapter 2}

attention to extreme performances when assessing recommenders, thus weight very good or very bad performances highly (Gershoff et al., 2003). The effect of extreme performances might differ with the order of their occurrence.

Second, in our study we investigated only short sequences of two successive recommendations. Future research could examine longer recommendation sequences and investigate whether the effect of relationship closeness on the use of performance information remains. As we showed that the total number of prior recommendations from the recommender did not influence the use of recent information, a myopic view of performance utilization over longer sequences appears plausible.

Third, this research focused on personalized recommendations in subjective product domains. Future research could investigate whether our results can be generalized to recommender evaluation in objective tasks that have nothing to do with personal preferences (e.g., buying stocks). This generalization is not trivial, as prior research suggests that consumers do not overstate friends' abilities in objective domains (Gershoff \& Johar, 2006). Therefore, we assume that the motivational account of performance information utilization would be dampened in such objective recommendation situations.

In conclusion, we offer an extended framework to examine how consumers utilize recommendation performance information. Our work links evaluation of recommenders based on their recent performance with the impact of relationships for this consideration. Our findings offer implications for consumers and companies. Taken together, the results provide a critical view of the evaluation of friends as recommenders. 


\section{Easing a Rough Start: How Relationships to Recommenders Affect Response to Service Failure in Initial Encounters (Paper 2)}

(With Yasemin Boztuğ $)^{3}$

To be submitted to Journal of Service Management.

Keywords: Service Failure, Recommendations, Relationship Closeness, Attribution,

Initial Encounters

\footnotetext{
${ }^{3}$ This paper was created in cooperation with the listed co-author. I was responsible for the literature review, the theoretical framework, the hypotheses development, the data management, the methodology, and the empirical analysis. My co-responsibilities comprised the positioning, the contribution statement, the conceptual framework, and the implication section.
} 


\section{Chapter 3}

\subsection{Introduction}

Service failures are service encounters that do not meet the expectations of customers and have severe consequences for customer loyalty (Voorhees et al., 2017). For example, after experiencing a service failure, customers often switch their service provider (Keaveney, 1995). Due to its managerial relevance, ample research has examined how the negative effects of service failure for companies can be mitigated. The two strategies service recovery and customer relationship management dominate this discussion. First, service recovery (e.g., Matos, Henrique, \& Rossi, 2007; McCollough, Berry, \& Yadav, 2000) describes actions a service provider takes towards dissatisfied customers as a response to service failure. Effective service recovery can compensate or oven overcompensate the negative consequences of service failure (Smith \& Bolton, 1998). Second, customer relationship management (e.g., Hess, Ganesan, \& Klein, 2003; Mattila, 2001; Sajtos, Brodie, \& Whittome, 2010) means building and maintaining strong relationships to customers that buffer undesirable response after service failure. Firm-customer relationships have been shown to protect service providers from the negative consequences of failure on customer loyalty (Hess et al., 2003).

However, for early stages of firm-customer relationships both strategies-service recovery and customer relationship management-appear to be impractical as remedies for service failure (Boulding, Kalra, Staelin, \& Zeithaml, 1993). On the one hand, if service failures occur in early relationship stages, companies had no time to establish customer relationships and develop strong bonds to customers (Berry \& Parasuraman, 2004). On the other hand, customers in early stages of a service relationship have been shown to be unlikely to voice out in case they are dissatisfied and thus a high share of service failures stays unnoticed while many customers defect "silently" (DeWitt \& Brady, 2003; Singh, 1990). While providers have less 
options in addressing service failures in the early relationship stage, such initial encounter ${ }^{4}$ service failure (hereafter: IESF) are exceptionally dramatic as they influence subsequent evaluations (Mattila, 2003) and customers have been shown to especially focus on reliability of a service during initial encounters (Berry \& Parasuraman, 2004). Thus, firms face a "double whammy" if service failures occur early in a relationship.

Surprisingly, despite its critical relevance for managers and consumers, we are unaware of research that has explored the response of customers to IESF and what can countervail such a situation. In this paper, we address these gaps. We examine customer response to failures in initial encounters when intent to complain is especially weak and firm-customer relationships are non-existent. Further, we examine the role of other consumers as a potential factor that may impact customers' response to IESF. Specifically, we suggest that whether a new customer uses the service as a result of a recommendation of a fellow consumer or not may affect how tolerant this new customer is when a service failure occurs in the initial service encounter. We also highlight that it matters who provides the recommendation. Our underlying rationale is that not only the relationship with a service provider, but also the relationship with another consumer that links the new customer to the firm influences the response to an ISEF. To test our reasoning, we examine whether relationship closeness of recommenders and new customers can to a certain extent replace a provider-customer relationship and thus constitute a "customer retention effect" of recommendations after IESF.

Recommendations among consumers have been widely discussed as a part of Word-ofMouth (WOM) literature (Berger, 2014). They can be regarded as a special form of WOM

\footnotetext{
${ }^{4}$ By initial encounter we mean the first interaction of a new customer with a service provider, for example when a customer visits a restaurant for the first time.
} 


\section{Chapter 3}

engagement as they are positive-valanced communication between a receiver and a recommender whom the receiver perceives as non-commercial, with regard to a brand, a product, or a service (Arndt, 1967) . Recent WOM research particularly centers on the additional value of WOM for companies beyond the acquisition of new customers (Kumar et al., 2010). This research emphasizes that customers are valuable to companies because of their social influence on other customers (Nitzan \& Libai, 2011). Specifically, studies have suggested that WOM might be superior to traditional marketing with regard to customer retention (Trusov, Bucklin, \& Pauwels, 2009). For example, Villanueva, Yoo, and Hanssens (2008) found that customers acquired through WOM have a higher lifetime value than customers acquired through traditional marketing like advertising. Similarly, research showed that incentivized referrals of other customers lead to longer firm-customer relationships in comparison to traditional marketing (Schmitt, Skiera, \& van den Bulte, 2011). However, this stream of literature does not inform how WOM influences perceptions of and response to service that failed. By measuring customer retention after multiple weeks up to three years (Schmitt et al., 2011; van den Bulte, Bayer, Skiera, \& Schmitt, 2018; Villanueva et al., 2008), prior research is also silent on the value of WOM in early stages of firm-customer relationships.

This paper extends the aforementioned previous work in that it examines the effectiveness of recommendations in specific, highly critical service contexts instead of considering the repercussions of WOM for service relationships in general or service retention of customers in later stages of the relationship. By doing so, we make several contributions. First, we are the first to focus on customer retention after "non-routine" services that have been inflicted by a service failure, which are particularly critical for firms. In doing so, this paper

\footnotetext{
${ }^{5}$ While the concept of recommendations is encapsulated in the broader concept of WOM, many authors use the term WOM when referring to recommendations. In this work we follow this approach and use the words recommendations and WOM interchangeably unless stated different.
} 
links the before-isolated literature streams of WOM and customer retention after service failure. Second, prior research analyzed the impact of WOM on service relationships with a longitudinal perspective to understand relationship development over time (Schmitt et al., 2011; van den Bulte et al., 2018; Villanueva et al., 2008). In contrast, our work is focused on the retention effect of recommendations after a critical event has spoiled the very beginning of a relationship. Third, prior work is based on data that does not contain detailed information of interrelations between acquired customers and their recommenders (van den Bulte et al., 2018). We instead use experimental data and explicitly consider relationships of customers to recommenders. Specifically, we are able to examine the differential effects of recommendations from relationally close and distant fellow consumers on reaction to service failure. We thereby pioneer in examining how relationships that involve other consumers (e.g., those who act as recommenders) shape the effects of service failure. On a more general level, we extend the prevalent dyadic customer-firm perspective when studying service relationships by considering the role of other actors beyond the focal customer (Alexander, Jaakkola, \& Hollebeek, 2018). Fourth, our approach also enables us to analyze potential psychological processes that drive the response to ISEF after recommendations from other consumers.

The remainder of the paper is structured as follows. The sections 3.2 and 3.3 develop a conceptual framework that considers the impact of service failure on customer loyalty, the particularities of initial encounters and the relationship to recommending fellow consumers as an influencing factor. The sections 3.4 to 3.7 discuss the methodology, data and results of our empirical studies. The paper concludes with a general discussion. 


\section{Chapter 3}

\subsection{Conceptual Background}

\subsubsection{Service Failure and Customer Loyalty}

Service failure heavily impacts customer behavior, which has led to considerable research attention (e.g., Hess et al., 2003; Mattila, 2004; McCollough et al., 2000; van Vaerenbergh, Orsingher, Vermeir, \& Larivière, 2014). For example, service failure has been shown to cause negative emotions of customers (Bonifield \& Cole, 2007; Kalamas, Laroche, \& Makdessian, 2008) and negatively affects their long-term satisfaction (McCollough et al., 2000; Smith, Bolton, \& Wagner, 1999). Ultimately, service failure lowers attitudinal and behavioral customer loyalty and translates into negative WOM and churn of customers (Keaveney, 1995; Mattila, 2004). Close firm-customer relationships buffer such negative effects of service failure and maintain loyalty towards a service provider despite service failure (Hess et al., 2003). Consequently, customers involved in committed service relationships have shown to be more tolerant when failures occur (Berry, 1995). Extant research suggest that this tolerance is built up by prior positive encounters with the service provider that lead to cumulated satisfaction over time (Bolton, 1998). This view is consistent with research that shows that the reaction to failures depend on a "reservoir of goodwill" (Germann, Grewal, Ross, \& Srivastava, 2014) that companies can fill up, e.g. by past service performance (Vázquez-Casielles, del RíoLanza, \& Díaz-Martín, 2007) or by strengthening their firm reputation (Hess, 2008). Bolton (1998) found that customers who have strong relationships with a provider weigh these priors more heavily than a single failure in a service encounter.

The protective effect of relationships at least partly stems from its influence on the way customers reason about service failure (van Vaerenbergh et al., 2014). According to attribution theory, when customers experience negative outcomes, they typically evaluate potential causes that may have led to the failure (Weiner, 1985). The result of this causal attribution process 
substantially impacts how customers react to failure (van Raaij \& Pruyn, 1998). In a service context, prior positive perceptions of service providers have been shown to motivate a more favorable reasoning about the causes that have led to service failure (Vázquez-Casielles et al., 2007). Two general questions determine causal attribution in the case of service failure: whether the service provider could have prevented the service failure (controllability attribution) and whether the service failure will likely occur in future interactions again (stability attribution, Weiner, 2000). Prior research highly suggests that customers' attributions after service failures are influenced by existing firm-customer relationships (Hess 2003). However, as there are no such priors at the beginning of service relationships, a favorable attribution seems unlikely then.

\subsubsection{Service Failure in Initial Encounters}

The development of relationships between organizations and customers is typically described by different stages (for overview see Zhang 2016). Several authors underline the special importance of the initial stage of interaction in a firm-customer relationship (Heide, 1994) which some authors also call exploration stage (Dwyer 1987, Zhang 2016). Early in a life cycle, customers collect information, form attitudes about the relationship partner and try to avoid failure (Heilman, Bowman, \& Wright, 2000). Similarly, Berry and Parasuraman (2004) found that customers early in a relationship are primarily tied to a firm through product or service performance. These findings illustrate that an initial encounter per se is a crucial touchpoint in a service relationship. If this initial encounter is as anticipated, it has little impact on the expectations of the customer an thus influences the trajectory of the service relationship only incrementally (Harmeling, Palmatier, Houston, Arnold, \& Samaha, 2015).

However, the first encounter is even more crucial as soon as service performance is outside the customer's "zone of tolerance" (Berry 1991). If the initial encounter is below prior 


\section{Chapter 3}

expectations it can be regarded a potentially transformational event that may dramatically change the trajectory of a relationship (Harmeling 2015). IESF are such events that substantially and negatively disconfirm prior expectations of the new customers. Research on turning points indicates that such an event may result in dramatic change in the evaluation of the relationship and may re-define the relationship to a relationship partner itself (Baxter \& Bullis, 1986). More dramatically, when service failure occurs in the first interaction with a customer, companies do not benefit from built-up goodwill from past interactions. This means customers cannot outweigh the failure experience with previous social bonds or positive experiences associated with the company. This is consistent with the finding that new customers have especially low intents to give service providers a second chance after a service failure (Bolton, 1998). To conclude, service failures seem particularly critical in initial encounters as there exist no prior firm-customer relationships in first interactions. Thus, new customers also cannot consider prior encounters that may influence their attribution in case of failure. In such a case it seems reasonable that customers look for other cues that surrogate the relationship to the service provider to guide their causal judgements about the background of a failure. Communication with other consumers may be one information source that consumers use to form expectations about companies prior to interactions (Bansal \& Voyer, 2000).

\subsubsection{Recommendations of Others and Response to Service Failure}

It is generally established that customers do not only develop favorable attitudes towards a company by encounters with that firm or through firm's marketing activities, but also through the communication with fellow consumers (Boulding et al., 1993). Prior research shows that recommendations of other consumers have a positive effect on service relationships (Trusov et al., 2009). Customers that have been brought to the company via referrals of existing customers usually exhibit higher margins and lower churn (Schmitt et al., 2011). Generally, prior research 
explains the effect of WOM on service relationships by two mechanisms: (1) better matching and (2) social enrichment (van den Bulte et al., 2018). Better matching means that other consumers usually know the service provider's offerings and the person they refer and thus are able to match both parties especially well. As a consequence, referred customers experience services tailored to their needs and thus are less likely to churn. Social enrichment refers to the social bonds between a customer and a service provider that may be strengthened by the presence of a recommender who is connected to both parties. Social enrichment leads referred customers to develop a stronger affective commitment to service providers (van den Bulte et al. (2018).

However, an IESF context with an immediate disappointment of expectations by service failure differs greatly from the context of prior studies as we pointed out before. Therefore, it is not clear whether the reported positive effects of WOM on service relationships can be transferred to an IESF setting. Drawing on the expectancy-disconfirmation paradigm, a conceivable alternative account would be that recommendations lead to higher expectations towards service providers and thus result in an especially disappointing experience in case of IESF. Similarly, research has shown that high expectations towards brands may even augment the negative effects of a product recall (Germann et al., 2014). Consequently, it remains unclear whether recommendations buffer or even amplify the negative effects of a service failure in an initial encounter. If recommendations have positive effects on response to service failure, it is still highly doubtable that the proposed mechanisms better matching or social enrichment are applicable for our context. First, IESF implies that a good match to the service provider cannot be assumed by the new customer. Second, it appears also unlikely that affective commitment to a service provider is developed when a failure occurs in the initial encounter. 


\section{Chapter 3}

\subsection{Hypothesis Development}

Based on the established buffering effect of close relationships between firms and customers on service failure response (Hess et al., 2003), we propose a novel mechanism which helps to explain the potential effect of recommendations on response to IESF. First, we suggest that also relationships to other actors than the firm may impact customer response to IESF. One group that is characterized by close relationships to new customers are other consumers. Specifically, close others often act as recommenders that bring new customers in contact with service providers (Nielsen, 2015). In general, several authors have acknowledged the pivotal role that relationships between consumers have for effectiveness of WOM (Duhan et al., 1997; Granovetter, 1973). Simpson, Griskevicius, and Rothman (2012) found that many consumption decisions are shaped by people with whom we have close relationships. Cavanaugh (2016) emphasized that choices are frequently influenced by actions or attitudes of close others like friends or family members. Relationship closeness reflects the emotional attachment between relationship partners (Swann \& Gill, 1997). This emotional attachment has also been shown to nurture beliefs about the suitability of recommenders (Gershoff \& Johar, 2006). For example, consumers commonly believe that friends are especially suitable in predicting their preferences (Eggleston, Wilson, Lee, \& Gilbert, 2015). This belief may also be salient when service failures occur after recommendations. However, research has also shown that individuals tend to overstate the ability of such strong-tie recommenders (Eggleston et al., 2015). This is because people are motivated to believe that close others know their preferences especially well (Gershoff \& Johar, 2006). The motivation to evaluate close others favorably is based on the need to protect relationships that are central to one's own self (Kenny \& Acitelli, 2001). Thus, a close relationship to the recommender could lead to stronger convictions that this person recommends only services that suits own preferences. Drawing on favorable beliefs about 
recommenders, we expect that a recommendation from another consumer may also influence a new customer's loyalty to a service provider that failed in the first instance. Further, we expect that the effect of recommendations on customer loyalty is qualified by the relationship of the recommender to the receiver of the recommendation. Therefore, we suggest that it is crucial for the response who recommends a service provider and that only a close relationship to a recommender may impact reactions to a service failure compared to traditional marketing. This leads to the following hypotheses:

Hla: Recommendations of close others (vs. traditional marketing acquisition) positively influence the loyalty towards a company after a service failure in the initial encounter.

H1b: Recommendations of distant others (vs. traditional marketing acquisition) do not influence the loyalty towards a company after a service failure in the initial encounter.

Drawing on attribution theory (Weiner, 1985), we suggest that a close relationship to the recommender may alter the causal reasoning of an occurring IESF in a way comparable to the effect that has been shown for close firm-customer relationships (van Vaerenbergh et al., 2014). This means that the effect of recommendations of others on IESF response may rely on reasoning towards the recommender which spills over to reasoning towards the service provider. Our rationale is that especially recommendations of close recommenders result in a more favorable attribution of service failure after IESF. One advantage of this approach is that it conceptualizes the effects of recommendations on a broader scope than prior research and considers relationships among multiple actors in the service ecosystem (Brodie, Fehrer, Jaakkola, \& Conduit, 2019).

Our premise is that the object of favorable attribution may not only be the close recommender, but that favorable attribution may also be transferred to the service provider that 


\section{Chapter 3}

is inevitably linked to the recommender. As a result, new customers that experience IESF because of a friends' recommendation may be inclined to use favorable attribution strategies towards the service provider. A favorable attribution of the service failure would be a way to reduce dissonance about the assumed suitability of the close recommender and the disappointing service failure experience. Drawing on Weiner (2000), this dissonance may influence reasoning in way that the failure was not fully the company's responsibility (controllability attribution) or that the failure was unsystematic and therefore will not likely occur in the future again (stability attribution). In the studied context this means that service failure may be attributed less controllable to the service provider and less stable when a customer has been referred by a close fellow customer (e.g., a friend) compared to a more distant fellow customer (e.g., an acquaintance). As a result, customers that have been referred by close others may show a higher intent to re-use the service compared to those referred by distant others because of shifts in controllability and stability attribution.

Thus, we expect that the customer retention effect of close others' recommendations is based on a shift in causal reasoning about the service failure. This mediated effect is comparable to the attribution effect of firm-customer relationships in case of service failure. Accordingly, we except the relationship closeness to be mediated by controllability and stability attribution. This leads to the following hypotheses:

H2a: The influence of relationship closeness on customer loyalty after IESF is mediated by lower attribution of controllability about the cause of the failure.

H2b: The influence of relationship closeness on customer loyalty after IESF is mediated by lower attribution of stability about the cause of the failure. 


\subsection{Overview of Studies}

We examined the influence of recommendations of close others on reactions to service failure empirically in a pilot study and two experiments. In the pilot study, we analyzed the extent to which bad recommendation performance of friends is inconsistent with individuals' expectations. This study served as a starting point to better understand the individuals' beliefs about friends as recommenders. In study 1, we investigated the potential effect of recommendations from relationally close and distant fellow consumers on the intent to retain with a service provider after an IESF. Finally, in study 2 we replicated our findings in another service domain and with a broader operationalization of consumer loyalty and also examined the underlying psychological process for the focal customer retention effect. We focused on stability and controllability attribution as potential accounts for the reduced response to service failure when the recommendation come from friends. Figure 4 illustrates our empirical approach. 
Figure 4: Effects of Recommendations from Distant and Close Others on Loyalty after IESF.

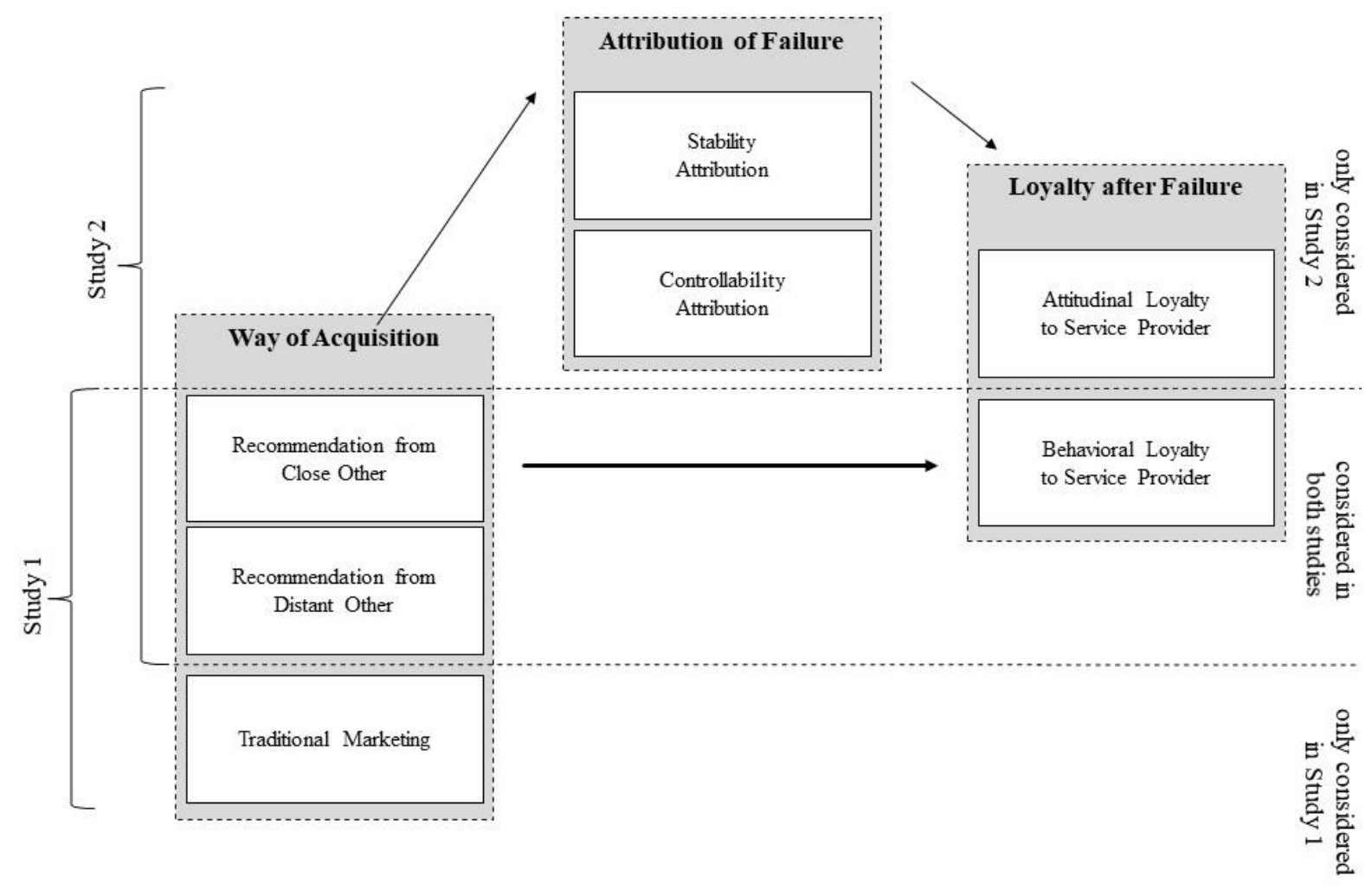

\subsection{Pilot Study}

In the pilot study, we examined individuals' beliefs about friends as recommenders and how these beliefs differ from those about recommenders with whom they are less involved (acquaintances). In addition, we were interested in determining how relevant the performance of friends and acquaintances as recommenders was to the participants. We regard differential beliefs in close and distant recommenders as a prerequisite for our reasoning that failed recommendations of friends are especially unexpected and therefore have a potential to arouse favorable attributions in the case of failure. 
In total, 65 university business students participated in the pilot study as part of a marketing course. Each participant answered four questions that asked about the perceived expectancy and relevancy of recommendation outcomes from friends and acquaintances (measured on 7-point scales, see Figure 5).

Figure 5: Belief in Friends and Acquaintances as Recommenders.

\author{
A: Expectancy of \\ Recommendation Outcomes
}

\author{
B: Relevancy of \\ Recommendation Outcomes
}
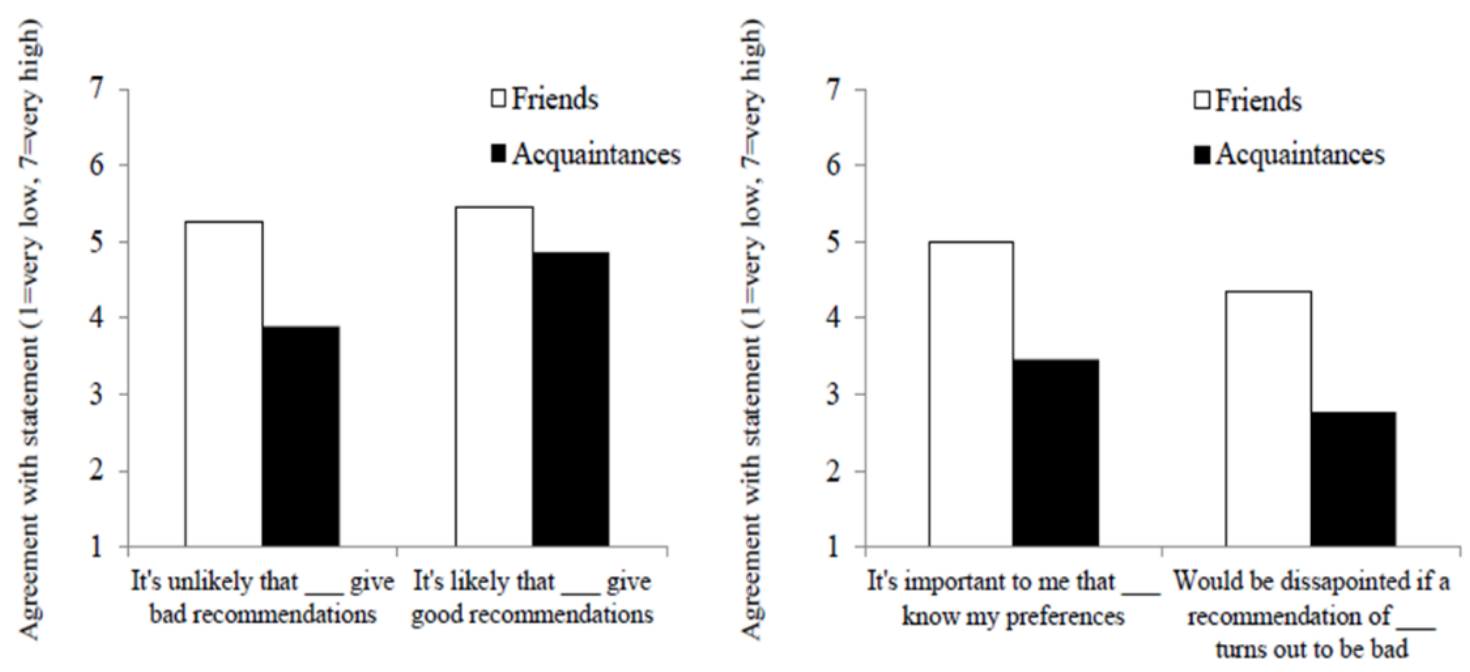

As expected, the participants assessed friends' likelihood to provide suitable recommendations as higher than those of acquaintances $\left(M_{\text {friends }}=5.45\right.$ vs. Macquaintances $=4.86$; $t(64)=4.39, p<.001)$. More important, the data suggest that the participants based their belief in friends as superior recommenders particularly on the expected absence of bad recommendations. Specifically, they deemed it more unlikely that friends (vs. acquaintances) would give bad advice $\left(M_{\text {friends }}=5.25\right.$ vs. Macquaintances $\left.=3.89 ; t(64)=7.58, p<.001\right)$. This is in line with our reasoning that people generally believe that friends very unlikely give bad advice. By contrast, the participants' beliefs about acquaintances as recommenders were vaguer. This 


\section{Chapter 3}

suggests that the participants regarded good and bad recommendations as plausible when the recommender was an acquaintance. Furthermore, our results indicate that recommendation performance of friends may be especially relevant to consumers. While the participants rated it important that friends know their preferences, whether acquaintances know their preferences or not meant less to them $\left(M_{\text {friends }}=5.00\right.$ vs. Macquaintances $\left.=3.46 ; t(64)=8.32, p<.001\right)$ Moreover, we found that bad recommendation performance of friends would disappoint participants more than bad recommendations of acquaintances $\left(M_{\text {friends }}=4.35\right.$ vs. Macquaintances $=2.77 ; t(64)=7.75$, $p<.001)$. Taken together, these results provide first support for our reasoning that bad performance of recommending friends is not consistent with expectations and thus has the potential to create dissonant feelings.

In study 1 , we transfer our rationale to a service failure context and examine the effect of recommendations from close and distant fellow consumers that lead to IESF in comparison to traditional marketing acquisition.

\subsection{Study 1: Service Failure at a Doctor's Appointment}

The goal of study 1 was to examine whether recommendations from other consumers influence a new customer's response to a service failure in an initial service encounter. In addition, we analyzed whether relationship closeness to the recommender reinforces the loyalty intentions towards the service provider after experiencing a service failure. Our analysis enables us to pin down the impact of recommendations and the role of relationships between consumers for the intent to re-consider a service provider that failed in the first place.

\subsubsection{Method}

Participants and Design. In total, 179 undergraduates and graduates recruited from Facebook groups of German universities completed the questionnaire. We randomly assigned the 
participants to one of three conditions in a single-factor between-subjects design (acquisition: recommendation from friend vs. recommendation from neighbor vs. traditional marketing). For data quality reasons (for details: see Appendix 2C), we excluded 20 participants from the original sample which resulted in a final sample of 159 participants (76\% female, $M=25.7$ years).

Procedure. Study 1 simulated an initial service encounter situation ${ }^{6}$ that either resulted from a recommendation of a friend or a neighbor and led to a service failure. These two conditions served as our manipulation of relationship closeness to the recommender. In a third condition, our reference, we stated that the initial service encounter was not based on a recommendation, but on traditional marketing. As service context we chose a doctor's appointment scenario with an impolite employee as service failure.

First, the participants were asked to imagine that they were in need for a new general practitioner as they felt ill and their previous general practitioner practice surprisingly was abandoned. In the 'friend' condition, we told the participants that a good friend recommended a general practitioner practice near their apartment. In the 'neighbor' condition, we framed this recommendation to come from a neighbor they had little to do with. Finally, in the 'traditional marketing' condition we asked the participants to imagine that they recognized an advertisement sign that led them to the new general practitioner practice near their apartment and that this practice seemed to fit their needs at first glance. In all three conditions, the participants imagined that they scheduled a doctor's appointment at the new practice for the next morning.

\footnotetext{
${ }^{6}$ Stimuli and measures of this study are provided in Appendix 2A.
} 


\section{Chapter 3}

Second, the participants imagined that when they arrived at the practice punctually on the next day, they had a negative incident with the practitioner assistant at the information desk. The description stated that the assistant welcomed them brusquely and blamed the participants to be too late as they needed to fill out several forms as a new patient and that this would take some time. In addition, we told the participants that the practitioner assistant made another derogatory remark about them in their presence. Unprompted employee actions are a common reason for failed services (Bitner et al., 1990) and therefore served as our service failure manipulation. At the end of the scenario, we told the participants that the actual doctor's treatment began as scheduled and briefly described it to be as expected.

As our target variable, we asked the participants whether they, based on their experience from the scenario, would retain or switch practice next time they needed to go to a general practitioner (measured on a 3-point scale: $1=\mathrm{I}$ would probably retain this practice, $2=\mathrm{I}$ would probably switch to another practice, $3=$ Not sure). As control variables, we measured how satisfied the participants were with the described service encounter (three items, measured on 7-point scales, adapted from Oliver \& Swan, 1989) and how severe they evaluated the service failure (three items, measured on 7-point scales, adapted from Sajtos et al., 2010). To ensure high data quality, we also asked whether the participants evaluated the scenario to be realistic (yes/no) and included an attention check asking how they were brought to the new practices (possible answers: recommendation of friend, recommendation of neighbor, traditional marketing). Next, the participants stated their demographics (age and gender). Finally, we asked whether the participants would complain after the IESF described in the scenario (measured on a 3-point scale: 1 = I would probably complain, $2=\mathrm{I}$ would probably not complain, $3=$ Not sure) and whether the participants had experienced a real IESF themselves within the last 12 months. 
Reliability checks confirmed the internal consistency of our controls 'satisfaction with the service encounter' $(\alpha=.83)$ and 'severity of the service failure' $(\alpha=.82)$. To check our manipulation of service failure, we further analyzed how the participants perceived the severity of the service failure. We found that on average the manipulated service failure was recognized as such and assessed as rather severe $(M=4.54, S D=1.35)$.

\subsubsection{Results}

To test our first two hypotheses, we compared the intent to retain with the practice between the three experimental conditions using Z-tests for column proportions. As indicated in Table 3, a significantly higher share of participants in the 'friend' condition (70\%) than in the 'traditional marketing' condition (44\%) stated to retain with the practice $(p=.008)$. The share of retainers in the 'neighbor' condition (61\%) was only marginal significantly different from the one in the 'traditional marketing' condition $(p=.082)$. Likewise, the share of participants that stated to switch practice was just about half as high in the 'friend' condition (19\%) as in the 'traditional marketing' condition (36.5\%) and significantly different from it $(p=.043)$. Answers from participants in the 'neighbor' condition did not differ from the 'traditional marketing' condition $(p>.1)$. Finally, the share of participants that answered to be not sure about retaining did not significantly differ between all conditions ( $p s>.2)$. 
Table 3: Effect of Way of Acquisition on Intent to Retain a New Practice After Initial Encounter Service Failure.

\begin{tabular}{|c|c|c|c|c|}
\hline \multicolumn{5}{|c|}{ Traditional } \\
\hline & & Marketing & Recommendation & Recommendation \\
\hline & & (Reference) & Neighbor & Friend \\
\hline & & $N=52$ & $N=54$ & $N=53$ \\
\hline \multirow[b]{2}{*}{ Retain } & $\mathrm{N}$ & $23 a$ & $33_{\mathrm{a}, \mathrm{b}}$ & $37_{\mathrm{b}}$ \\
\hline & $\%$ & $44 \%$ & $61 \%$ & $70 \%$ \\
\hline \multirow[b]{2}{*}{ Not Sure } & $\mathrm{N}$ & $10_{a}$ & $8 \mathrm{a}$ & $6 a$ \\
\hline & $\%$ & $19 \%$ & $15 \%$ & $11 \%$ \\
\hline \multirow{2}{*}{ Switch } & $\mathrm{N}$ & $19 \mathrm{a}$ & $13_{\mathrm{a}, \mathrm{b}}$ & $10_{\mathrm{b}}$ \\
\hline & $\%$ & $36 \%$ & $24 \%$ & $19 \%$ \\
\hline
\end{tabular}

Note: Values in the same row for which the subscript is not identical differ at $\mathrm{p}<0.05$ in the two-sided test for equality for column proportions.

As our primary interest was whether the recommendations from other customers would make participants retain more likely with a service provider after IESF, we collapsed the answers 'switch' and 'not sure' which led to a binary retention variable (coded 1 for 'probably retain', 0 for 'probably not retain or not sure'). We used binary logistic regression to estimate the effect of a recommendation from 'friend' or a 'neighbor' versus 'traditional marketing' on the probability to retain with a service provider. As independent variables we computed two dummy-coded variables that indicated the conditions 'neighbor' and 'friend' in reference to 'traditional marketing'. While we found a significant positive effect of a recommendation of a friend $(b=1.070, S E=.41, p<.001)$ compared to traditional marketing, a recommendation from a neighbor again only marginally affected the intention to stick with a service provider in comparison to traditional marketing $(b=.68, S E=.40, p=.083)$.

Next, we included our controls 'satisfaction with the service encounter' and 'severity of the service failure' as additional independent variables into the model. Our rationale was to rule 60 
out the potential alternative explanation that differences in retention were caused by differential perceptions of the service failure. This could have been plausible as previous research has shown that individuals eventually interpret ambiguous outcomes in a way that they are consistent to their prior expectations (G. Cohen, Aronson, \& Steele, 2000; Lord \& Taylor, 2009). If the effects we observed in study 1 were really produced by biased perception of service failure, the explanatory value of the dummy-coded variables 'neighbor' and 'friend' should be mitigated when incorporating 'satisfaction' and 'severity'. However, after extending the model as described, the significant effect of recommendations from friends and the insignificant effect of recommendations from acquaintances compared to traditional marketing in the original model remained (for details: see Appendix 2D).

Finally, we examined the relevance of IESF as crucial events for companies. Therefore, we analyzed how prevalent IESF was for individuals. The majority of the participants (74\%) stated that they had at least once experienced an IESF themselves in the last 12 months. This result backed our reasoning that IESF is a common situation for customers. Next, we analyzed the participants' intent to complain after the IESF described in the scenario. More than half of the participants (59\%) stated they would probably not complain. This backed our reasoning that IESF often remain silent and thus are a hidden problem for companies.

\subsubsection{Discussion}

Our first study showed that IESF is a challenge for companies that may result in immediate defection of new customers. Our results indicate that many consumers seem to experience IESF regularly, but do not complain to service providers. Study 1 also showed that recommendations of fellow consumers have a customer retention effect after IESF. This effect was clearly apparent when consumers had been referred by a close friend compared to traditional marketing. We interpret this as support for our $\mathrm{H}_{1 \mathrm{a}}$ consistent with our prediction that customers 


\section{Chapter 3}

are more loyal to a service provider after IESF when they have been referred by a close fellow consumer than when they are acquired by traditional marketing methods. However, recommendations of weak-ties seem to have a less substantial impact in comparison to traditional marketing than recommendations of friends, indicated by only marginal difference in intent to retain. This provides preliminary support for our $\mathrm{H}_{1 \mathrm{~b}}$ that a distant relationship to the recommender is a factor that lowers the loyalty effect of recommendations.

\subsection{Study 2: Service Failure in a Restaurant}

The results of study 1 demonstrated that recommendations from fellow consumers reduce behavioral response to services failure in the area of practice retention after an incident with an impolite service employee. Yet the following question arises: Can this effect be generalized to service failures in other domains and for other types of service failures? Study 2 was designed to strengthen the generalizability of our findings. Therefore, in study 2 we examined another service domain that is frequently object to service failure and where recommendations among customers are common, the restaurant domain. In our second study, we also simulated a service failure that differed from an impolite service employee. Instead of a service failure that was based on employee action (Bitner et al., 1990), in study 2 we examined another prevalent failure: extensive waiting time.

In study 1 , we concentrated on differential effects of recommendations from close and distant others in comparison to traditional marketing. In the second study we "zoomed in" to potential differences response that may occur because of relationship closeness to the recommender. Therefore, the second study focused on the differential effects of relationally close versus distant recommenders. In study 1 we have manipulated the relationship closeness to the recommender in an abstract way. The participants were told to imagine a situation with a person who was not specified by name and just introduced by his or her relationship. This 
may have led to a weak mental representation of the recommender. To make the mental representation of the recommender more realistic, in study 2 we manipulated relationship closeness with the recommender by asking the participants to think of a real friend (acquaintance) of them who would act as the recommender in the scenario and to type this person's name. We referred to this name in the questionnaire several times. We also chose to measure our target variable in a way that it would captured attitudinal and behavioral aspects of customer loyalty. While study 1 concentrated on the behavioral loyalty towards the service provider, the multidimensional conceptualization of customer loyalty in study 2 was supposed to make our results more comprehensive and thus better comparable to previous work in service research (Mattila, 2004; Zeithaml, Berry, \& Parasuraman, 1996). Finally, in study 2 we examined the suggested underlying psychological mechanism to explain the customer loyalty effect of recommendations. Specifically, we tested whether the effect stems from a shift in in causal reasoning of the service failure expressed by differential stability or controllability attribution.

\subsubsection{Method}

Participants and Design. In total, 137 participants recruited from an online student panel completed the questionnaire. We randomly assigned the participants to one of two conditions in a single-factor between-subjects design (acquisition: recommendation from friend vs. recommendation from acquaintance). Due to data quality reasons (for details: see Appendix $2 \mathrm{G})$, we excluded 19 participants from the original sample which resulted in a final sample of 118 participants (64\% female, $M=24.1$ years).

Procedure. After the introduction, we asked the participants to think of either one of their real friends or real acquaintances that they could generally imagine to provide a restaurant 


\section{Chapter 3}

recommendation. Next, the participants were told to state this person's name and to describe their relationship in up to three words. This served as our manipulation of relationship closeness to the recommender. Then, the participants were asked to read a scenario where they coincidently met their friend (acquaintance). We told the participants that while chatting, they received a phone call from their parents who liked to come for a visit at in the next days and suggested to go out for dinner in a restaurant of the participant's choice. The participants were told they would have no idea for a suitable restaurant and asked their friend (acquaintance) for advice. In turn, the friend (acquaintance) recommended a little Italian restaurant nearby that the participants did not know yet. The participants were asked to imagine that they reserved a table in this restaurant.

In the next phase of the scenario, we described the subsequent restaurant visit that included a long waiting time as service failure. First, we asked the participants to imagine that their parents arrived for the visit and they went to the recommended restaurant together. We told the participants that the restaurant made a good general impression, but was unexpectedly filled. This information was included so that participants could envision both controllable and uncontrollable causes for long waiting (Hess et al., 2003). To manipulate a service failure, the participants were told that they had to wait $60 \mathrm{~min}$. after ordering before they received their dishes. The scenario ended with a statement that, when the dishes were finally served, they were delicious and authentic.

We measured the participants' loyalty towards the service provider on five items adapted from (Zeithaml et al., 1996). Three of the five items were related to WOM intentions and two items referred to the intended use of the service in the future (measured on 7-point Likert scales). After the participants stated their assessment of customer loyalty to the restaurant, controllability and stability attributions were measured with regard to the service 
failure. For this purpose, we adapted the attribution scale of Hui and Toffoli (2002). Three of the items related to the control attributions and another three items to the stability attributions of the service failure (measured on 7-point scales). The failure attribution was purposely measured subsequent to the loyalty measurement to prevent distortions of our target variable.

The relational closeness with the recommender was measured on four items adapted from Swann and Gill (1997): the time spent doing things with the recommender, conversing with the recommender, sharing problems and worries with the recommender, and their liking of the recommender (measured on 7-point semantic differentials). Also, we asked on two items to what extend the participants think they share a taste with the recommender and how consciously they think the recommender reflected before recommending the restaurant (both measured on 7-point Likert scales). Finally, we measured attention checks and control variables similar to study 1 .

Reliability checks confirmed the internal consistency of our measurements of customer loyalty $(\alpha=.93)$, severity $(\alpha=.77)$, satisfaction $(\alpha=.85)$, controllability attribution $(\alpha=.82)$, and stability attribution $(\alpha=.86)$ First checks indicated that our manipulations worked as intended: Relationship closeness was higher in the friend condition than in the acquaintance condition $\left(M_{\text {friend }}=6.17\right.$ vs. $\left.M_{\text {acquaintance }}=3.24 ; t(116)=19.64, p<.001\right)$. Also, the service failure was recognized as such and judged as rather severe $(M=4.75, S D=1.29)$. Thus, it was ensured that the described service failure situation was not trivial for the participants. There were no differences in severity perception between both experimental groups $\left(M_{\text {friend }}=4.65 \mathrm{vs}\right.$. $\left.M_{\text {acquaintance }}=4.85 ; t(116)=-.854, p>.3\right)$. 


\section{Chapter 3}

\subsubsection{Results}

Loyalty Towards the Service Provider. We analyzed the predicted effect of relationship closeness of the recommender on loyalty towards the service provider with an independent twosample t-test. As expected, participants that had been recommended by a friend were more loyal to the service provider after IESF than those that had been recommended by an acquaintance $\left(\mathrm{M}_{\text {friend }}=<4.48\right.$ vs. $\left.M_{\text {acquaintance }}=3.64 ; t(116)=3.81, p<.001\right)$. This result supports our premise that loyalty towards a service provider after IESF is impacted by the relationship closeness to a recommender.

Parallel Mediation Model. Next, to test our $\mathrm{H}_{2 \mathrm{a}}$ and $\mathrm{H}_{2 \mathrm{~b}}$, we analyzed the proposed explanations for the diverging effect of recommendations from friends and acquaintances. Therefore, we conducted a parallel mediation analysis to examine how relationship closeness influences the response to IESF. We used PROCESS model 4 (5000 bootstraps, Hayes, 2018) to test the proposed parallel mediation. The indirect effect through controllability attribution was not significant (95\% CI: -.01 to .01). However, there was a significant indirect effect of relationship closeness on loyalty through stability attribution ( $b=.07 ; 95 \%$ CI: .02 to .13$)$. As the direct effect of relationship closeness on PI $(b=.10, p=.04)$ was significant as well, the findings indicate partial mediation. These results were also robust when we incorporated the measured control variables to our mediation analysis (for details: see Appendix $2 \mathrm{H}$ ).

\subsubsection{Discussion}

Study 2 corroborates the results from study 1 and extends our main findings to another service domain and another service failure type. In addition, the results of study 2 confirmed that stability attribution reduces the response of new customers to service failure when they have been referred to the service provider by friends. This result shows that people perceive service failure less systematic after a recommendation of a friend. In turn, the favorable stability 
attribution reinforces willingness to give the service provider a second chance. However, we could not find support for our contention that recommendations of close others make people more well-disposed regarding the controllability of service failure. This means, while a recommendation of strong-tie makes it more likely that consumers assume that the failure will not happen again in the near future, the responsibility perception of the failure is not affected.

\subsection{General Discussion}

\subsubsection{Theoretical Contributions}

This research examines how recommendations from other consumers influence customer response to IESF. We especially concentrated on the role of relationship closeness to the recommender on customer loyalty towards the service provider after IESF.

Our results demonstrate that IESF regularly happen and are therefore a relevant topic of investigation. Surprisingly scant research has focused on initial interactions of service providers with customers and how the impact of negative first impressions can be reduced for companies. Our results support and extends prior research that has highlighted the importance of these initial encounters (Heath, Chatterjee, Basuroy, Hennig-Thurau, \& Kocher, 2015; Mattila, 2003).

We also extend what is known about company strategies to overcome consequences of service failure in initial encounters. While research has established that firm-customer relationships act as a buffer (Hess et al., 2003), we show that in initial encounters also the acquisition by recommendations of other consumer protects firms from negative consequences. This finding specifies the premise that WOM is valuable for companies beyond the acquisition of new customers (Kumar et al., 2010) and supports the general rationale that customer acquisition and retention are interdependent processes (Thomas, 2001). In the paper we 


\section{Chapter 3}

examine recommendations of other customers as one factor that might decrease churn and negative WOM after experiencing service failure in first encounters.

We show that not all types of recommendations have the same beneficial effect. Our results suggest that the relationship between the recommender and the receiver of the recommendation plays an important role for the response to IESF. This is an important extension of prior research on WOM in service contexts that has not distinguished between weak-tie and strong-tie recommenders yet (Schmitt et al., 2011; van den Bulte et al., 2018).

Furthermore, we investigate how exactly close relationships impact the response to service failure. One finding is that customers have favorable beliefs about recommenders they feel closely related to. Specifically, it is inconsistent to them that close others would recommend services that do not meet their preferences or are unsuitable. Supported by our empirical data, we propose a shift of stability attribution as a novel mechanism to account for the customer retention effect of strong-tie recommendations after IESF. This psychological process substantially differs from the previously examined accounts for the beneficial effect of WOM on service relationships (van den Bulte et al., 2018).

In short, our research integrates two literature streams, WOM literature and service failure literature. We extend what is known about the role of relationship closeness and WOM for service failure in several ways: We show that the way that a customer been brought to the service provider, tremendously determines his reaction to service failure. We also show that recommendations lower negative response like churn towards service providers. Our work is different from previous papers in that we especially focus on the first contact with a service provider. Thus, our results add to the understanding of IESF and how companies can reduce the negative consequences of IESF. 


\subsubsection{Public Policy and Managerial Implications}

Our research has direct implications for consumer welfare. For example, close others are not always as informative in predicting preferences as individuals might believe (Eggleston et al., 2015). After consumers realize that the first encounter with a service did not turn out well, they are more likely to attribute this information favorably and in consequence re-consider this service when they have come to the service by a recommendation of a close other. This may result in sticking with unreliable service providers. However, giving service providers a second chance after IESF must not always mean that consumers take bad decisions. For example, if a service failure occurs on an initial encounter with a service provider that otherwise always offers consistently good service quality, consumers can even benefit from this behavior.

In addition to better understanding consumer decision making, our findings may be helpful for companies. In this paper we suggest a novel value driver of customer recommendations for companies: retention of other customers after service failure. This seems especially valuable for companies in domains where service failure happens regularly. Our findings suggest that close relationship recommenders have a special relevance for companies and that their value does not only encompass high likelihood to acquire new customers. New customers, which are a vital necessity for business success, moreover seem to become more well-minded towards failure as a result of recommendations by close others. Our research calls for incorporating these benefits into company assessment of customer value. Specifically, companies should consider the protective effect of close recommenders as an additional component of a customer's engagement value (Kumar et al., 2010). Finally, our findings underline the importance of encouraging existing customers to generate company-related WOM especially to strong-ties, for example by tell-a-friend campaigns. 


\section{Chapter 3}

\subsubsection{Avenues for Further Research}

Further research could complement our findings in several ways. First, the focus on the detrimental role of initial service encounters deserves more attention as it sets the tone for subsequent service encounters and is the basis of building relationships to service providers (Mattila, 2003). Often consumers may not be willing to use a service for a second time after initial service failure. Our results suggest that many customers face first-impression failures regularly. In such situations, a recommendation may immediately influence reasoning of consumers and affect subsequent decision making.

Second, we limited our experiments to organic WOM, thus recommendations that occur naturally. However, more and more companies use financial incentives for recommenders to actively seed WOM transmission. Yet, our results do not account for the potential differences between WOM that occurs naturally and WOM stimulated by companies. Another future research opportunity would be to determine the joint effect of relationship closeness of recommenders and incentives on customer loyalty after service failure. Of special interest would be the question whether incentives act as a boundary condition for the customer retention effect of recommendations. In other words: Do people also give a service provider a second chance after a service failure that was based on an incentivized recommendation of a friend? This generalization is not trivial, as prior research suggests that consumers do infer ulterior motives when incentives are involved (Wirtz, Orsingher, Chew, \& Tambyah, 2013). Therefore, we assume that the beneficial effect of relationship closeness would be dampened in such recommendation situations.

Finally, we introduced a perspective that concentrates on the impact of relationships between recommenders and recommendation receivers on customer retention. However, we think that examining the relationship of the recommender to the service provider is another 
relevant avenue for future research. In our manipulations we introduced this relationship as established (the recommender had used the service several times before). It would be fruitful to also examine whether the effects of strong relationships to recommenders we found fade away when the recommender's relationship to the service provider is just lose. For example, this would be the case when friends recommend a service they have just started to use themselves or even have not used yet.

\subsubsection{Conclusion}

To summarize, we offer a framework that helps to examine the role of relationships to recommenders in an IESF setting. Thus, this paper provides insights into how WOM can help to save service relationships that went wrong after acquisition. This paper is one of the first to address a mechanism that links recommendations of close recommenders to services that failed in the initial service encounter. Our findings offer implications for consumers and companies. Taken together, the results extent the understanding of initial service encounters, recommendations from close others, and their value for marketers. 
This page is intentionally left blank. 


\section{Conducting Mediation Analysis in Marketing Research (Paper 3)}

(With Steffen Jahn and Yasemin Boztuğ $)^{7}$

This version of the paper was submitted to Marketing ZFP in May 2017 and has been accepted for publication in Marketing ZFP, published by Beck as:

Demming, Carsten Leo, Steffen Jahn, \& Yasemin Boztuğ (2017): Conducting Mediation Analysis in Marketing Research, Marketing ZFP, 39 (3), 76-93.

https://doi.org/10.15358/0344-1369-2017-3-76

Keywords: Recommendations; Agent Evaluation; Motivated Reasoning; Relationship Closeness; Word-of-Mouth; Recency Bias

\footnotetext{
${ }^{7}$ This paper was created in cooperation with the listed co-authors. Steffen Jahn and I equally contributed to this paper. I was responsible for the literature review, the theoretical foundation, and the data example. My coresponsibilities comprised the positioning, the contribution statement, and the implication section.
} 


\section{Chapter 4}

\subsection{Introduction}

Researchers in marketing and other fields are often interested in the causal effect of a predictor on an outcome variable. Mediation analysis adds to the understanding of such an effect by examining how well the effect can be explained by another variable, called a mediator (Iacobucci, 2008). Thus, mediation analysis reveals how the predictor indirectly affects the outcome through the mediator. A prominent marketing example comes from research on advertising effectiveness, which revealed that the effect of ad liking on purchase intent is mediated by brand liking (MacKenzie, Lutz, \& Belch, 1986). Mediation analysis is therefore especially relevant for those interested in grasping the underlying mechanism of a focal effect (Preacher, 2015).

While common as a concept, an actual methodology of how to analyze mediation had not been established until Baron and Kenny (1986) proposed causal steps a researcher should apply to support a mediation hypothesis. The causal steps approach is based on the idea of inferring mediation from a series of separate regression models (Baron \& Kenny, 1986; James \& Brett, 1984; Judd \& Kenny, 1981). Another approach for analyzing mediation that was becoming increasingly popular at the time is structural equation modeling (SEM, Bagozzi \& Yi, 1988; Bollen, 1989). Due to its ability to simultaneously estimate all model paths (Iacobucci, Saldanha, \& Deng, 2007), SEM is superior to the causal steps method. However, as its relative sophistication poses a number of problems and pitfalls (J. Anderson \& Gerbing, 1988), there have been calls for alternatives that are easy to use while also being equivalent to SEM.

Recent developments indicate that regression-based bootstrap approaches could be that alternative (Preacher \& Hayes, 2004, 2008). In particular, sophisticated mediation analysis has been simplified by Hayes' provision of the mediation analysis macro PROCESS, which is available for SPSS and SAS (Hayes, 2017), as well as the accompanying textbook (Hayes, 
2013). Due to these advances, regression-based mediation analysis now allows the same reliability in estimation as SEM does (Hayes \& Scharkow, 2013). Therefore, applications of regression-based mediation analysis with novel methodology (Hayes, 2013; Preacher \& Hayes, 2004, 2008) have been garnering increasing interest. The growing number of citations of the respective methodology papers reflects the relevance of mediation analysis as a means of theory development and testing in marketing research (see Figure 6). Figure 6 displays how many times the most influential mediation publications (i.e., Baron \& Kenny, 1986; Hayes, 2013; Preacher \& Hayes, 2004, 2008; Sobel, 1982) have been cited in the top-tier marketing outlets Journal of Consumer Research, Journal of Consumer Psychology ${ }^{8}$, Journal of Marketing, Journal of Marketing Research, and Marketing Science since the publication of Baron and Kenny's (1986) causal steps approach. As can be seen from Figure 6, interest in mediation analysis has increased substantially in the last decade.

Although there is now a well-established body of literature advancing the methodology of mediation analysis since Baron and Kenny (1986), this technical literature has not fully "diffused to practicing researchers" (Zhao, Lynch, \& Chen, 2010, p. 197). As a result, researchers often diverge in how they conduct tests of mediation (Hayes \& Scharkow, 2013). This paper contributes to existing literature by concisely integrating theoretical and practical knowledge in order to help in applying mediation analysis. Our main goal is to provide an overview of what mediation analysis means and which approaches exist to establish mediation, followed by a tutorial that demonstrates how to apply the state-of-the-art methodology. In the tutorial we illustrate how to specify, interpret, and report results using PROCESS (Hayes, 2017). The analyses are conducted with examples from the marketing context.

\footnotetext{
${ }^{8}$ Because consumer researchers frequently use mediation analysis, we added the Journal of Consumer Psychology to our list of top-tier journals.
} 
Figure 6: Citation Trend of Influential Mediation Analysis Publications.

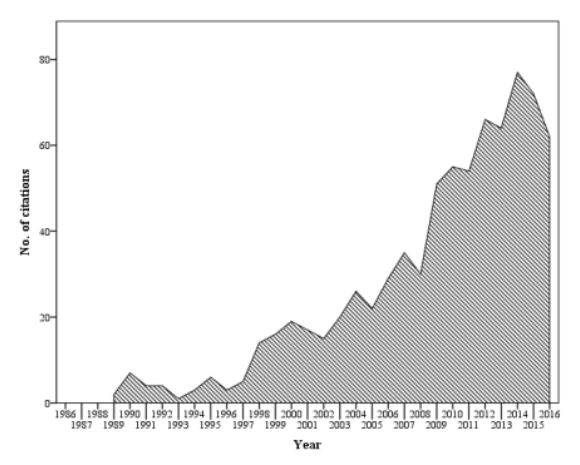

\subsection{Characteristics of Mediation}

Central to the concept of mediation is the so-called mediator. Extending a simple causal inference where a predictor $X$ causes an outcome $Y$, the mediator $M$ intervenes within this relationship. A mediator $M$ is therefore a variable that is influenced by the predictor $X$ and in turn influences the outcome $Y: X \rightarrow M \rightarrow Y$. When conducting mediation analysis, the researcher is primarily interested in this intervention process, namely the indirect effect, because it reveals 
something about how the causal relationship works (Iacobucci, 2008). Therefore, examining the indirect effect is the focal element of theory testing with mediation analysis (Hayes, 2013).

However, to estimate the extent to which the mediation process explains the relationship between the predictor $X$ and the outcome $Y$, it is also necessary to consider the so-called direct effect. The direct effect represents the causal influence of $X$ on $Y$ that is not explained by the mediator $M$ (James \& Brett, 1984). As we will outline in Section 2.3, examining the direct effect is particularly useful for further theory building (Zhao et al., 2010).

\subsubsection{Indirect Effect: Key to Establishing Mediation}

In a first step, a researcher is often interested in whether a proposed mediator $M$ can explain an effect of $X$ on $Y$ at all. This question is addressed by estimating the indirect effect through the mediator $M$ (Hayes, 2013). Hence, interpreting the indirect effect is the foundation for inference about a mediation hypothesis (Baron \& Kenny, 1986). In the following, we discuss how the indirect effect can be interpreted. It is important to note that the interpretation of the indirect effect is dependent on the model structure, especially the number of variables in the model and their interrelationships. While there is a multitude of possible mediation models, in this paper we focus on four prototype model groups (Hayes, 2013). Three of these model groups entail mediation only; hence we refer to them as models of "pure" mediation: (i) simple mediation, (ii) parallel mediation, and (iii) serial mediation. Aside from these "pure" mediation model groups, there are models that additionally contain moderator variables, referred to as moderated mediation models. Figure 7 illustrates the prototype model groups. 
Figure 7: Typology of Mediation Model Groups.

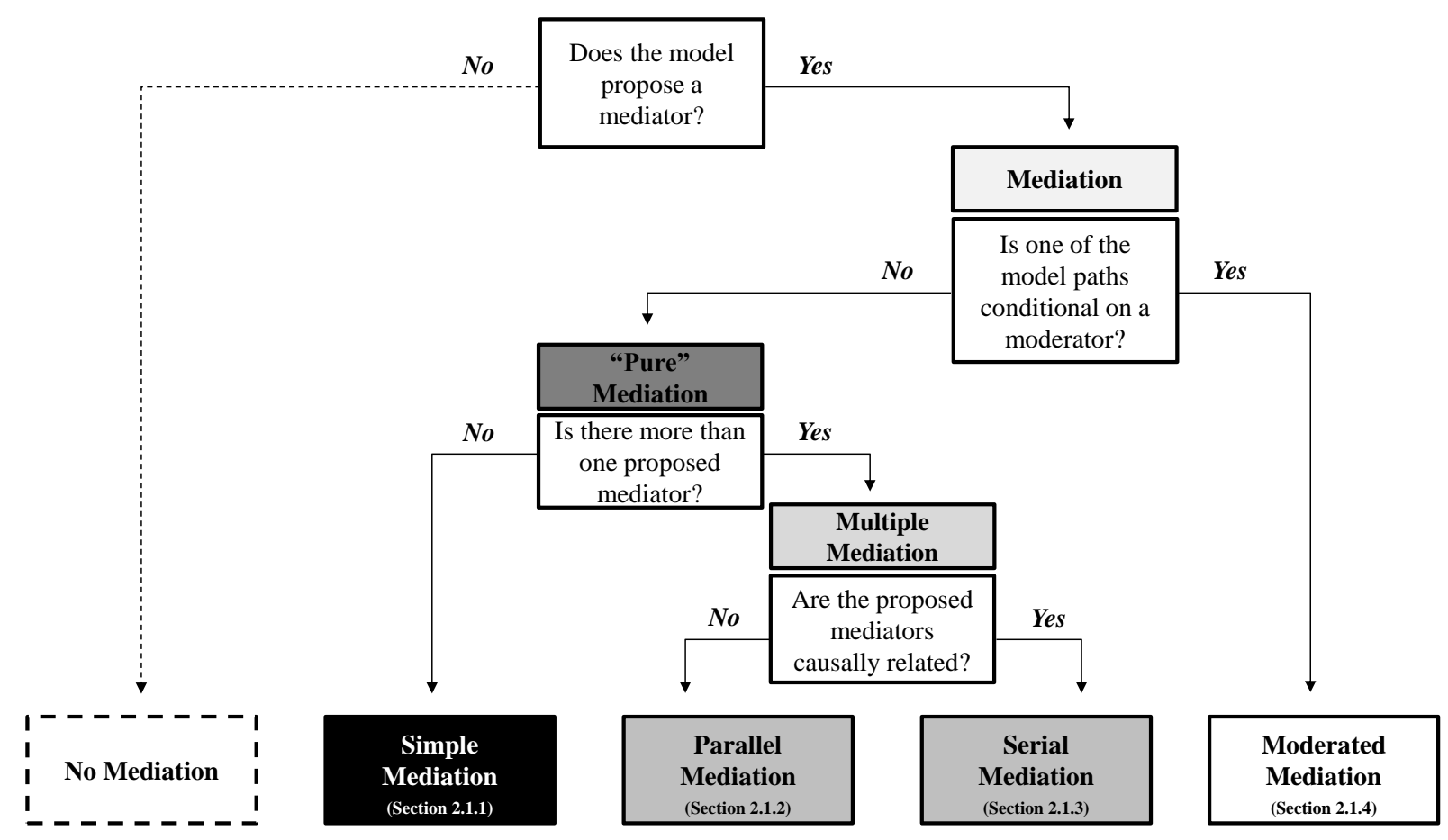

Simple mediation captures the standard $X \rightarrow M \rightarrow Y$ causal system, which means that there is exactly one mediator. In case of two or more mediators in the model, one can speak of multiple mediation (Hayes, 2013). If the multiple mediators are causally unrelated, this is called parallel mediation, while serial mediation is present if at least two of the mediators in the model are causally related (i.e., one mediator affects another one and they form a causal chain). Moderated mediation means that at least one mediation path is linearly dependent on another variable. Each mediation model group is discussed in greater detail in the following section.

\section{Simple Mediation}

Mediation extends simple regression by introducing an explaining variable, the mediator (see Figure 8). When there is exactly one mediator $M$ intervening in the causal relationship of $X$ on $Y$, this is called simple mediation. Conceptually, simple mediation means that a change in $X$ 
leads to change in $M$ (path $a$ ), and that change in $M$ leads to change in $Y$ (path $b$ ). The indirect effect is depicted as path $a b$ because it is the product of the two paths that connect the predictor $X$ to the mediator $M$ (path $a$ ) and the mediator $M$ to the outcome $Y$ (path $b$ ). If the indirect effect $a b$ is greater or smaller than zero (i. e., if it is statistically significant), one can claim that some form of mediation takes place (Zhao et al., 2010).

\section{Figure 8: Simple Mediation Model as an Extension of a Simple Causal Relationship.}

\section{Simple regression}

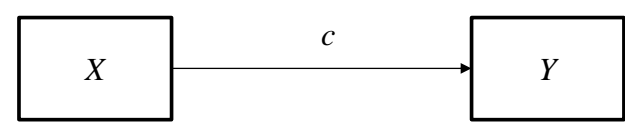

$X$ : predictor variable

$M$ : mediator

$Y$ : outcome variable

Simple mediation

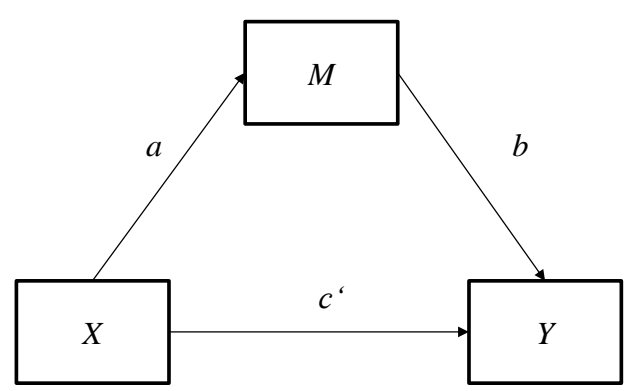

$a$ : effect of $X$ on $M$

$b$ : effect of $M$ on $Y$

$a b$ : indirect effect of $X$ on $Y$

$c^{\prime}$ : direct effect of $X$ on $Y$

$c$ : total effect of $X$ on $Y$

$=a b+c^{\circ}$

(based on Preacher and Hayes, 2004, p. 718)

Simple mediation is the most basic form of mediation and allows one to make inferences about the underlying mechanism that connects an independent with a dependent variable. If the underlying process involves more than one mediator, so-called multiple mediation models are used. We discuss two forms of multiple mediation - parallel and serial mediation - in the following sections. 


\section{Chapter 4}

\section{Parallel Mediation}

In some cases, there are alternative theories to explain an effect of $X$ on $Y$. In such cases, investigating the role of only one mediator is not enough. For example, while one theory might propose a mediator $M_{1}$, another theory might propose a different mediator $M_{2}$ for the same relationship (Hayes, 2009). Considering two or more mediators that are not causally interrelated is the most basic extension of the simple mediation model; it is called parallel mediation (Hayes, 2013). Parallel mediation models enable researchers to probe different mediation theories simultaneously in a model (e.g., Guevarra \& Howell, 2015). The example of two mediators would lead to a conceptual model structure like the one shown in Figure 9.

\section{Figure 9: Parallel Mediation Model with Two Mediators.}

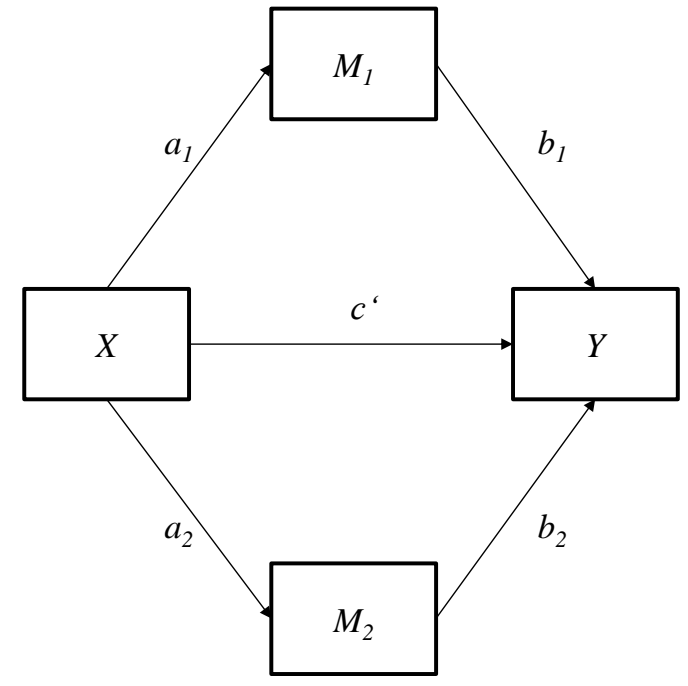

$X$ : predictor variable

$M_{1}:$ mediator 1

$M_{2}:$ mediator 2

$Y$ : outcome variable

$a_{1} b_{1}:$ specific indirect effect of $X$ on $Y$ through $M_{1}$ $a_{2} b_{2}$ : specific indirect effect of $X$ on $Y$ through $M_{2}$

$c$ ': direct effect of $X$ on $Y$ total indirect effect: $a_{1} b_{1}+a_{2} b_{2}$

(based on Hayes, 2013, p. 126)

In models with more than one mediator, several specific indirect effects exist that can be attributed to one of the mediators. In the example displayed in Figure 9, there are two specific indirect effects $a_{1} b_{1}$ and $a_{2} b_{2}$. If the aim of the researcher is to compare these two mediation processes, it is useful to assess the importance of each specific indirect effect. To do so, the researcher could check which of the proposed parallel mediations (i. e., specific indirect effects) 
is significant and then compare the magnitude of those specific indirect effects by testing whether they are equal in size (Preacher \& Hayes, 2008).

All specific indirect effects sum up to the total indirect effect, which expresses the extent to which all mediators together can explain the relationship between $X$ and $Y$. We note that there are cases in which specific indirect effects with different signs cancel each other out, leading to an insignificant total indirect effect, despite having significant specific indirect effects (Rucker, Preacher, Tormala, \& Petty, 2011). Such a finding would be valuable, as it identifies two antagonistic mechanisms and thus offers deeper insight into the relationship between $X$ and $Y$ (Hayes, 2009). This implies that finding a non-significant total indirect effect does not automatically mean that the conceptual model is flawed.

\section{Serial mediation}

Whenever the researcher hypothesizes that two or more mediators in a model influence each other, this is called serial mediation. In contrast to parallel mediation, serial mediation means that the mediators themselves are in a hierarchical causal relationship. Serial mediation is especially useful for investigating fine-grained causal chains of mediation (Hayes, 2013) and is commonly employed in the marketing field (e.g., Hur, Koo, \& Hofmann, 2015; Winterich \& Zhang, 2014). Figure 10 depicts an example in which one mediator affects another mediator. 
Figure 10: Serial Mediation Model with Two Mediators.

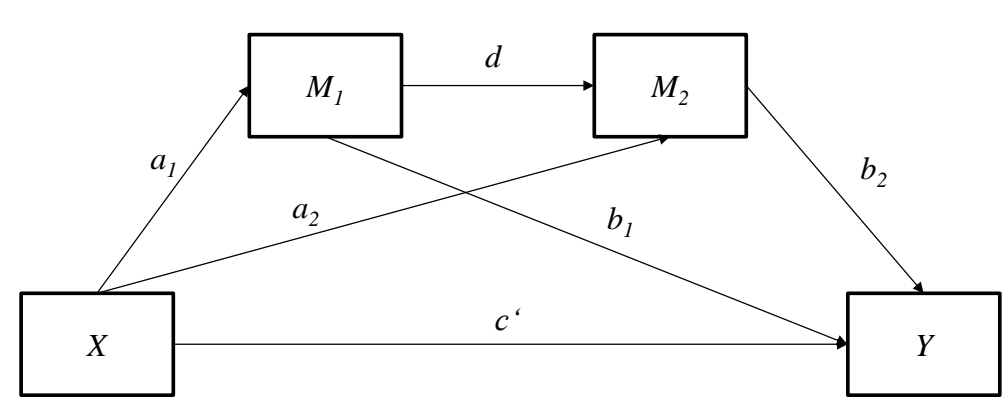

\author{
$X$ : predictor variable \\ $M_{1}$ : mediator 1 \\ $M_{2}$ : mediator 2 \\ $Y$ : outcome variable \\ $a_{1} d b_{2}$ : long-way specific indirect effect \\ of $X$ on $Y$ through $M_{1}$ and $M_{2}$ \\ $a_{1} b_{1}$ : shortcut specific indirect effect \\ of $X$ on $Y$ through $M_{1}$ only \\ $a_{2} b_{2}$ : shortcut specific indirect effect \\ of $X$ on $Y$ through $M_{2}$ only \\ $c$ ': direct effect of $X$ on $Y$ \\ total indirect effect: $a_{1} d b_{2}+a_{1} b_{1}+a_{2} b_{2}$
}

(based on Hayes, 2013, p. 145)

Similar to parallel mediation, the indirect effect in a serial mediation model is split up into several specific indirect effects. In the two-mediator example, three specific indirect effects can be distinguished. First, there is the long-way mediation, which involves both mediators: $a_{1} d b_{2}$. The long-way mediation represents a causal chain of mediators and is therefore the foundation of the serial mediation hypothesis. Second, there are two shortcut mediations, which each involve only one mediator: $a_{1} b_{1}$ and $a_{2} b_{2}$. If the long-way mediation is significant, serial mediation can be claimed. If the long-way mediation is not significant, this indicates that one of the other forms of mediation is more likely: if both shortcut mediations are significant, this indicates parallel mediation (as in Figure 9); and only one significant shortcut mediation indicates simple mediation (as in Figure 8). As in parallel mediation, the sum of all indirect effects constitutes the total indirect effect. The total indirect effect indicates the extent to which the long-way and all shortcut mediations together explain the effect of $X$ on $Y$. 


\section{Moderated mediation}

Researchers often are not only interested in detecting a particular process (which would be tackled by a "pure" mediation analysis) but also want to investigate the conditions under which this process is active (e.g., Blanchard, Carlson, \& Hyodo, 2016). Examining such conditions (also called boundary conditions of the focal effect) offers valuable information that helps assess whether indirect effects are conditional on different groups of respondents, contexts, or - more generally - on another variable (Preacher, Rucker, \& Hayes, 2007). For example, a proposed mediation might exist for one subgroup of the sample but not for another subgroup. Aside from this example of switching the mediation on and off, the so-called moderator variable might also strengthen or weaken the mediation or switch the mediation's direction (represented by a change in sign).

Whenever the mediation process is dependent on another variable, this is called moderated mediation (James \& Brett, 1984; Muller, Judd, \& Yzerbyt, 2005). Moderated mediation analysis works similarly to moderated regression analysis, with the exception that an indirect effect is altered. In moderated mediation, the moderator influences either one or both of the two paths of the indirect effect ( $a$ and $b$; Hayes 2013). Most moderated mediation models propose that the moderator alters the relationship of $X$ on $M$ (so-called first-stage moderated mediation, panel A of Figure 11). However, it is also possible that the moderator conditions how the mediator $M$ influences the outcome variable $Y$ (so-called second-stage moderated mediation, panel B of Figure 11). Furthermore, one or more moderators could also impact both paths of the indirect effect (panels C and D of Figure 11). 
Figure 11: Selection of Variants of a Moderated Mediation Model.

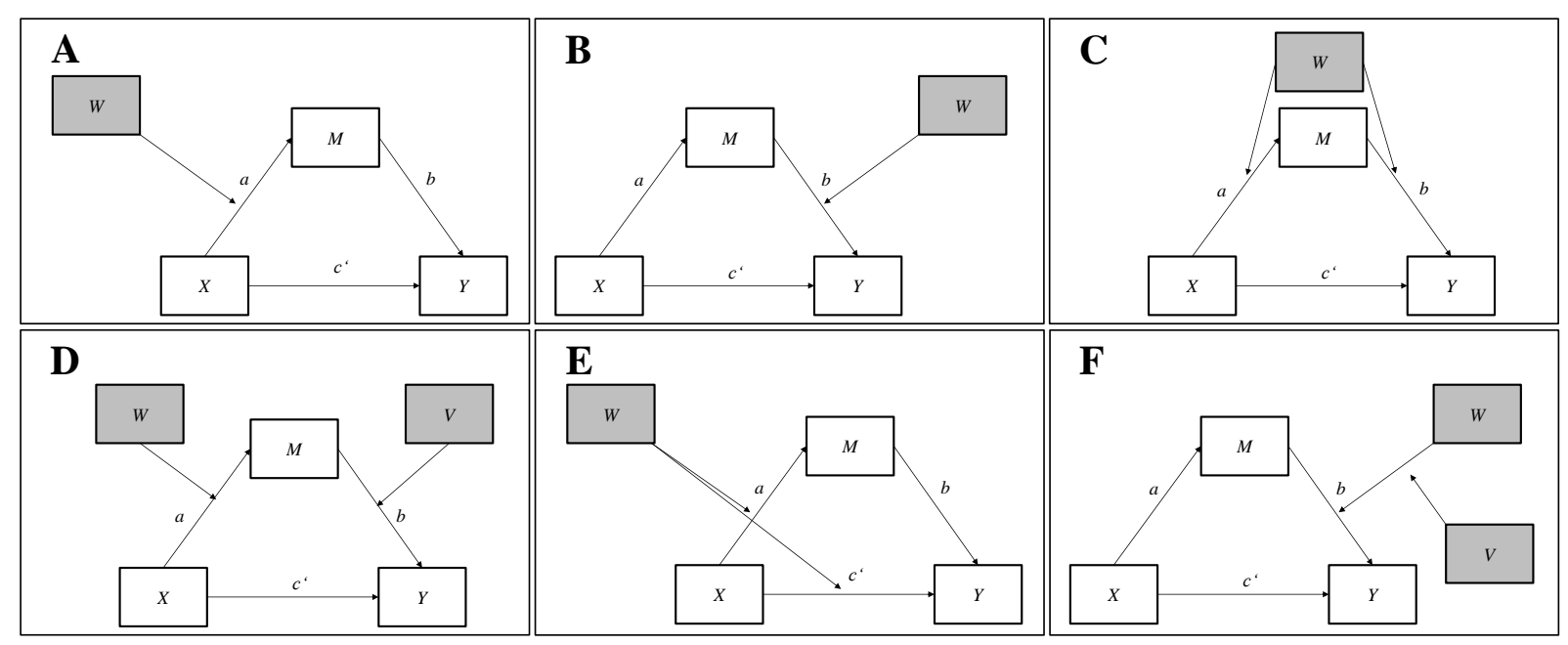

(own illustration based on Hayes, 2013, p. 14)

The influence of the moderator is not necessarily limited to the indirect effect and can include the direct effect (panel E of Figure 11). Further extensions, such as higher-order interactions (panel F of Figure 11), are also possible. The myriad of potential combinations makes it necessary to reason a priori about conditional processes and develop a model based on the specific theorizing.

As moderated mediation is about inferring whether an indirect effect is linearly conditioned by a moderator, the most central result of such a model would be the so-called conditional indirect effect of $X$ on $Y$ (Iacobucci, 2008). Although many authors have conceptually referred to moderated mediation (Baron \& Kenny, 1986; James \& Brett, 1984; Muller et al., 2005), an appropriate procedure for examining a conditional indirect effect was offered only recently (Hayes, 2015). The procedure involves a formal test of the conditional nature of proposed mediators called the index of moderated mediation (Hayes, 2015). 


\subsubsection{Direct Effect: Key to Assessing the Importance of the Mediation}

While a significant indirect effect of $X$ on $Y$ through $M$ answers the question of whether a proposed mediation exists, a researcher might also be interested in understanding to what extent the mediator can explain the relationship between $X$ and $Y$ (Rucker et al., 2011). In mediation analysis, this is determined by the direct effect of $X$ on $Y$, which represents the influence of $X$ on $Y$ that is unrelated to change in $M$. Given a significant indirect effect but an insignificant direct effect, the mediation fully explains the variation of $Y$ by $X$. In this case, researchers speak of full mediation (Baron \& Kenny, 1986; Zhao et al., 2010). However, if the direct effect is significant, the mediator $M$ only partially explains the effect of $X$ on $Y$ and the term partial mediation is used (James \& Brett, 1984; Zhao et al., 2010). While some authors claim full mediation to be the gold standard, most articles that use mediation analysis report only partial mediation (Iacobucci, 2008). In the following, we examine how distinguishing partial from full mediation may offer implications for theory building.

\subsubsection{Mediation Types and Their Implications for Theory Building}

Zhao et al. (2010) developed a typology of mediation based on the interpretation of the indirect and direct effects. Figure 12 illustrates this approach of distinguishing different mediation types (including non-mediation) and the associated implications for theory building.

As previously stated, a significant indirect effect combined with an insignificant direct effect reflects full mediation (Baron \& Kenny, 1986). In the case of full mediation, the underlying process is adequately captured and there is no need to search for additional explanatory elements (e. g., another mediator). In contrast, partial mediation implies that the researcher could find other mediators that have thus far been omitted in the analysis (Rucker et al., 2011). Following Zhao et al. (2010), the partial mediation concept is divided into two subtypes: complementary partial mediation and competitive partial mediation. Complementary 


\section{Chapter 4}

partial mediation occurs when the indirect effect and the direct effect have the same sign. This means that there could be another potential mediator with the same sign as the existing mediator "hidden" in the direct effect. In contrast, competitive partial mediation takes place when the indirect effect and the direct effect have opposing signs. Competitive partial mediation implies that the "hidden" potential mediator and the existing mediator have opposing signs (Zhao et al., 2010).

Figure 12: Mediation Types and Their Implications for Theory Building.

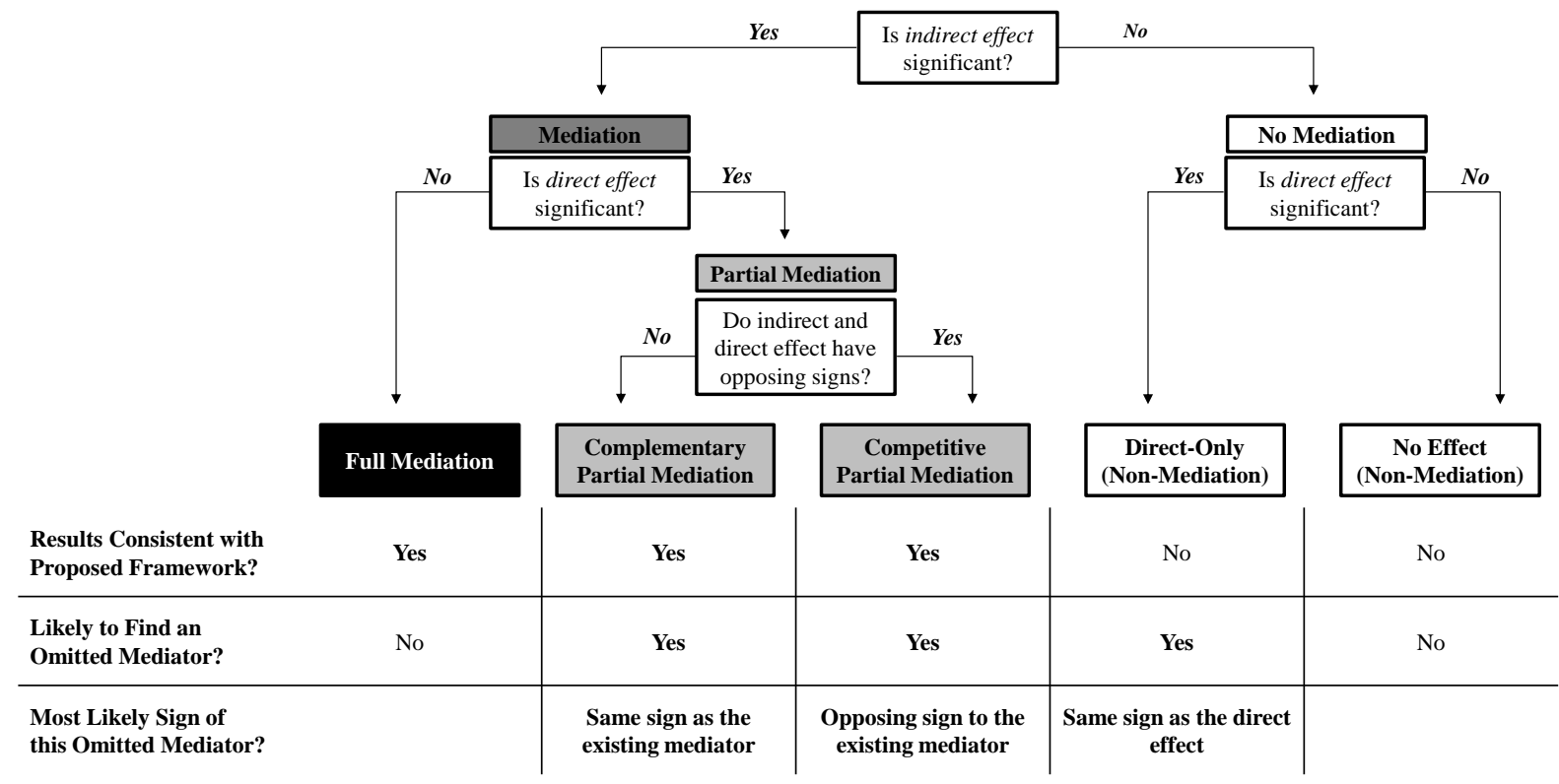

(own illustration based on Zhao et al., 2010, p. 201)

Besides pointing to omitted mediators, partial mediation may also indicate that an important moderator has not been taken into account (Shrout \& Bolger, 2002). This could mean that the proposed mediation might only apply for a certain group or under a certain condition (omitted moderated mediation). If the moderator is not considered, there is a risk of underestimating the importance of the mediation process (Shrout \& Bolger, 2002), such as inferring partial mediation when in reality there is full mediation. 
An insignificant indirect effect suggests non-mediation (Zhao et al. 2010). In this case, the same conclusions as before apply: if the direct effect is significant, there is a chance that the true mediator has been omitted. We note that in such a case, examination of paths $a$ and $b$ is particularly informative. If path $a$ or $b$ is not significant or very small in magnitude, this could explain the insignificance of the indirect effect as a whole, and it can guide future modification of the inconsistent conceptual framework. If both the indirect and direct effects are insignificant, $X$ and $Y$ are apparently unconnected.

\subsection{Approaches to Examine Mediation}

In the marketing field there are three dominant approaches to examining mediation: the causal steps method proposed by Baron and Kenny (1986), the normal theory approach introduced by Sobel (1982, 1986), and the regression-based bootstrapping approach put forth by Preacher and Hayes $(2004,2008)$. While the first two approaches are often used in conjunction and represent the traditional way of testing for mediation, bootstrapping is a more recent approach in mediation analysis.

\subsubsection{Traditional Approaches}

Traditionally, the most influential approach in probing mediation has been the causal steps approach. Though Judd and Kenny (1981) as well as James and Brett (1984) already discussed the technique, it was finally proposed by Baron and Kenny (1986) and is therefore known as the Baron-and-Kenny approach (Kenny, 2008). The basic principle of the causal steps approach is that it does not test the indirect effect itself, but logically infers mediation from testing all paths of the model separately in four steps ${ }^{9}$ (Baron \& Kenny, 1986). The approach involves analyses of (i) the total effect of $X$ on $Y$, (ii) the effect of $X$ on $M$, (iii) the effect of $M$ on $Y$, and

\footnotetext{
9 In their work, Baron and Kenny (1986) describe three regression equations and thus three steps. Because the third equation is used to draw two different inferences, we refer to them as separate steps.
} 


\section{Chapter 4}

(iv) the direct effect of $X$ on $Y$ (see Table 4). Baron and Kenny (1986) propose that one can only claim mediation if all effects in the first three steps turn out to be significant. Given this prerequisite, one can claim full mediation if the direct effect in the fourth step is non-significant and partial mediation if it the direct effect is smaller than the total effect.

Table 4: Steps of the Baron-and-Kenny Approach.

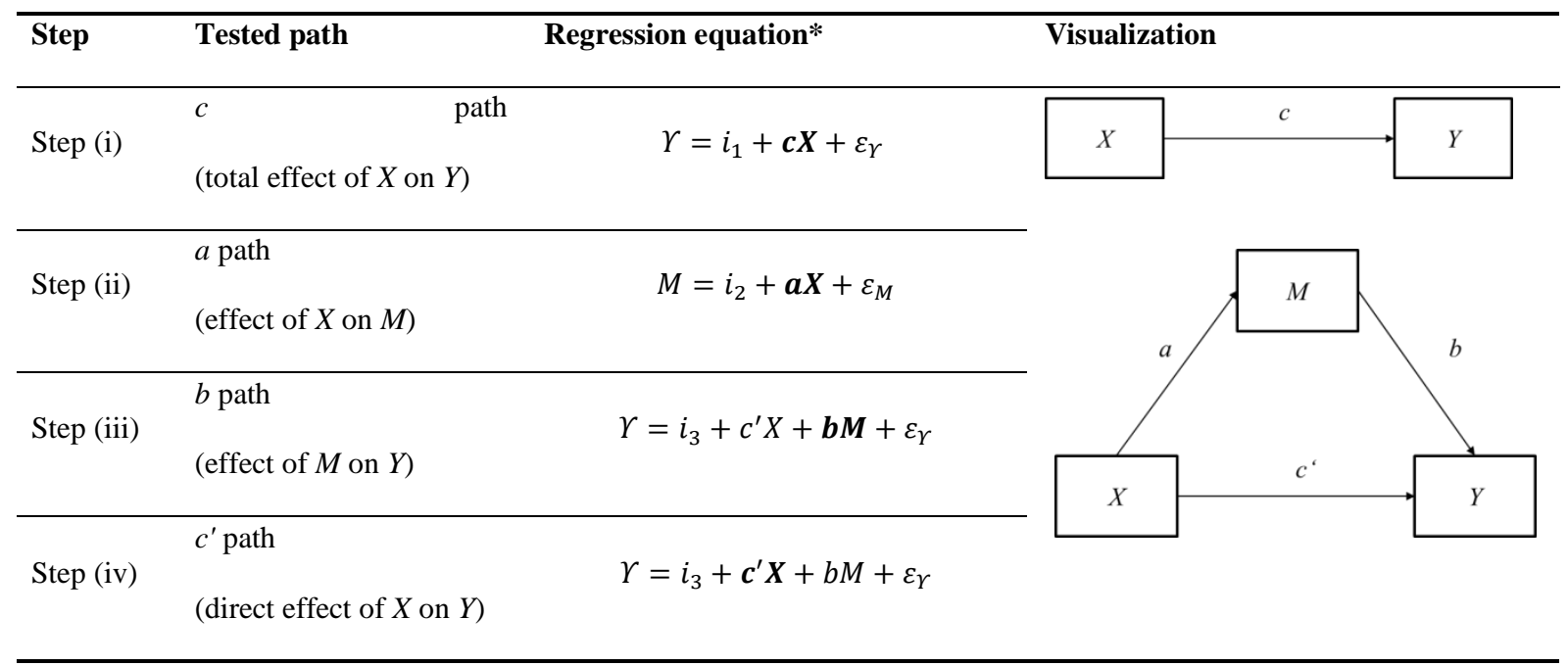

Notes: * Bold terms symbolize the tested parameters.

(own illustration based on Müller, 2009, p. 247)

The Baron-and-Kenny approach has been criticized for several reasons. The most critical issues are its lack of power (which means that it often cannot uncover a genuine mediation process) as well as its failure to test the indirect effect $a b$ (Hayes, 2013; MacKinnon, Lockwood, Hoffman, West, \& Sheets, 2002; Preacher \& Hayes, 2004, 2008). In addition, the requirements for steps (i) and (iv) seem overly restrictive and are unnecessary for establishing mediation (Hayes, 2009; Rucker et al., 2011; Zhao et al., 2010). Due to these limitations, the Baron-and-Kenny approach no longer seems to be recommended (Hayes, 2013; MacKinnon et al., 2002). 
The normal theory approach (Sobel, 1982, 1986), also called the Sobel test, addresses one weakness of the Baron-and-Kenny approach by establishing a formal test of the indirect effect $a b$. The Sobel test uses a logic for the inference of the indirect effect similar to that usually used for the estimation of direct effects. To conduct the test, one calculates the product of the coefficients $a$ and $b$, divides this product by an estimate of the standard error of $a b, s e_{a b}$, and compares the outcome to a critical value from the standard normal distribution (Sobel, 1982):

$$
Z=\frac{a b}{s e_{a b}}
$$

Several methodological variations of the Sobel test exist, each varying in the way that $s e_{a b}$ is estimated. The simplest estimation approach (Baron \& Kenny, 1986; Sobel, 1982), also referred to as first-order delta solution, encompasses the squared coefficients $a$ and $b$ and their squared standard errors:

$s e_{a b}=\sqrt{a^{2} s e_{b}^{2}+b^{2} s e_{a}^{2}}$

More complex estimation approaches include an additional product term of both squared standard errors, with this term either added to Equation 2 (Aroian, 1947; so-called second-order delta solution) or subtracted (Goodman, 1960; so-called unbiased delta solution). As all methods yield very similar results (Hayes \& Scharkow, 2013; MacKinnon, Warsi, \& Dwyer, 1995), the simple first-order delta solution seems to be the most straightforward approach. Nevertheless, the following remarks hold for all variants of the Sobel test.

The Sobel test's shortcomings result from its normality assumption of the sampling distribution in the indirect effect $a b$. This assumption is usually only met in very large sample sizes (i. e., $n>1,000$; Kisbu-Sakarya, MacKinnon, \& Miočević, 2014), while in smaller samples the sampling distribution of $a b$ tends to be asymmetric (Bollen \& Stine, 1990; Stone 


\section{Chapter 4}

\& Sobel, 1990). Although this limitation becomes less problematic with increasing effect sizes (e. g., a sample size of $n=100$ seems sufficient to detect medium-sized mediation effects; MacKinnon et al., 2002), the Sobel test has specific weaknesses in detecting mediation when either path $a$ or path $b$ is weak. Therefore, the Sobel test has low power in detecting indirect effects and thus tends to be overly conservative. This means that the Sobel test might indicate that there is no indirect effect, while in reality there is mediation (Shrout \& Bolger, 2002). Hence, there is some risk of overlooking a genuine indirect effect in the data when using the Sobel test, unless the effect size or sample size is large (for details regarding mediation effect size and required sample size, see Fritz \& MacKinnon, 2007). Due to these limitations, use of (any variant of) the Sobel test is not recommend (Hayes \& Scharkow, 2013).

\subsubsection{Bootstrapping Approach}

Bootstrapping is a non-parametric approach that bypasses the problem of questionable distributional assumptions of traditional techniques and enables an accurate test of the indirect effect (Bollen \& Stine, 1990; Shrout \& Bolger, 2002), even in small samples (Preacher \& Hayes, 2008). Importantly, bootstrapping provides more power in detecting indirect effects, but it does not show a higher type-I-error tendency (i. e., claiming mediation although there is none) than the traditional methods (Hayes \& Scharkow, 2013). Because one can easily employ bootstrapping for mediation analysis via macros such as PROCESS (Preacher \& Hayes, 2004, 2008), the approach is being applied increasingly.

Bootstrapping relies on resampling of the data (Efron, 1982), whereby one draws a large number (e. g., 10,000) of new samples of size $n$ with replacement from the original sample. The model parameters are estimated for each new sample, resulting in a large number of estimates for each parameter. The estimates can then be ordered by size to draw a probability density 
distribution for each path parameter (Preacher \& Hayes, 2008). Figure 13 shows a hypothetical example for such a density distribution of the indirect effect $a b$.

\section{Figure 13: Example of a Bootstrapped Sampling Distribution of the Indirect Effect $a b$.}

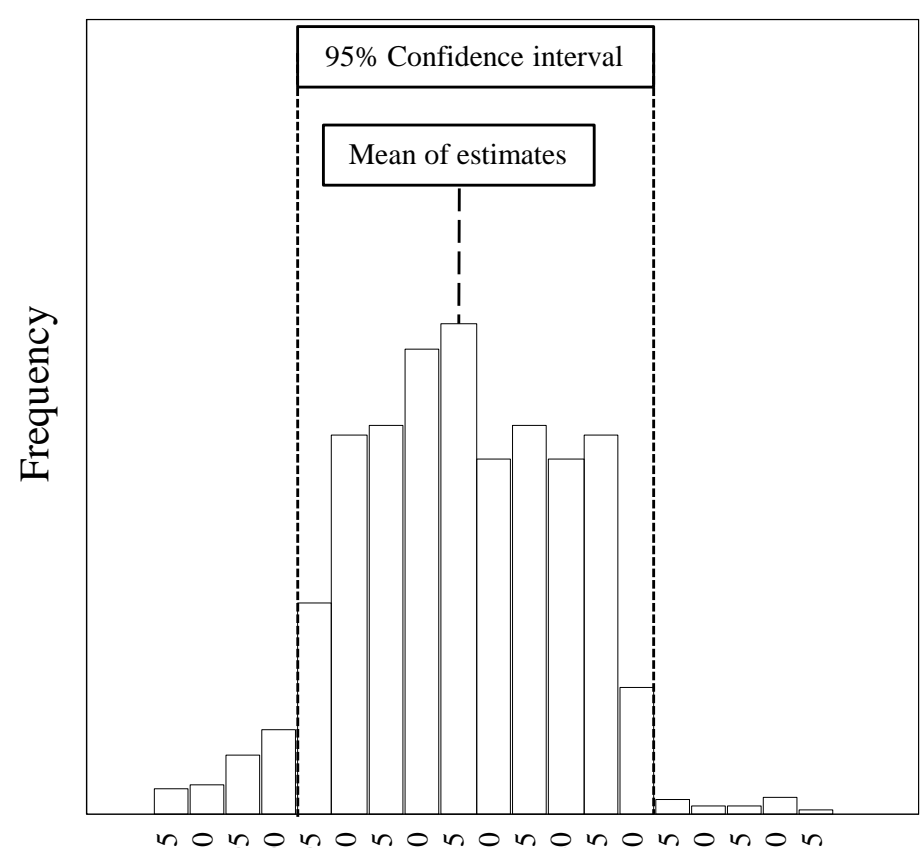

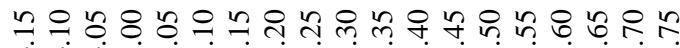

Coefficients of indirect effect $a b$

(own illustration based on Preacher \& Hayes, 2004, p. 721)

The mean of all bootstrap estimates is calculated for the point estimate of the indirect effect $a b$ (see Figure 13). Because a non-normal distribution of parameters precludes the calculation of $t$ - and $p$-values, the significance of the indirect effect $a b$ is inferred from the confidence interval of its bootstrap distribution. If the confidence interval does not include zero, one can be statistically confident that the effect is different from zero.

In the basic form, called percentile bootstrap, the confidence interval is determined by two percentile cutoffs of the sampling distribution (e. g., $2.5 \%$ and $97.5 \%$ in the case of $\alpha=$ $.05)$. In the example of 10,000 bootstraps illustrated in Figure 13, the $250^{\text {th }}$ highest $(.05)$ and 


\section{Chapter 4}

$9751^{\text {st }}$ highest score $(.50)$ define the $95 \%$ confidence interval. The results of the percentile bootstrap in Fig 8 indicate that the proposed indirect effect is significantly different from zero, as the confidence interval does not include zero (meaning it does not encompass positive and negative values). Therefore, one can say with $95 \%$ confidence that mediation is present (Preacher \& Hayes, 2004).

The percentile bootstrap is especially suitable in circumstances where robustness of the estimation is important, such as when samples include potential outliers (Creedon \& Hayes, 2015), when either the $a$ or $b$ path is large and the other path is zero (Koopman, Howe, Hollenbeck, \& Sin, 2015), or when facing small sample size $(n<50$; Koopman et al., 2015). In case of larger sample size it is recommendable to use an alternative form called bias-corrected bootstrap. This procedure generally results in slightly more liberal bootstrap confidence intervals because it adjusts the confidence interval for bias in the bootstrap sample distribution (Efron, 1987). Such bias may result from non-symmetric bootstrap sample distributions and is not accounted for by the percentile bootstrap (Efron \& Tibshirani, 1993). The differences across forms of bootstrapping are usually small, but they can sometimes influence the inference. While percentile bootstrapping may be reasonable in adverse situations (such as small sample size), bias-corrected bootstrap today is the standard form in mediation analysis.

Although percentile bootstrap and bias-corrected bootstrap differ slightly in their estimates, both outperform the Sobel test and Baron-and-Kenny approach remarkably with regard to statistical power (Hayes \& Scharkow, 2013) and propensity to type I error (MacKinnon et al., 2002). Hence, among the methods that are commonly used, reliable, and easy to conduct, bootstrapping seems to be the most promising approach for mediation analysis. After two seminal papers and add-ons about the bootstrapping approach in mediation analysis (Preacher \& Hayes, 2004, 2008), Hayes (2013, 2017) released a macro for SPSS and SAS called 
PROCESS, which combines the functionality of the preceding add-ons. In the following, we demonstrate how to conduct and interpret mediation analysis following the bootstrapping approach with PROCESS. The tutorial illustrates the specification of the different model groups and the interpretation of respective results with an example from advertising effectiveness.

\subsection{Tutorial: Estimating Mediation Models with PROCESS}

PROCESS (which can be downloaded from the developer's website; Hayes, 2017) is specialized for mediation analysis, moderation analysis, and combinations of both procedures using the regression-based bootstrapping approach. PROCESS provides a dialog box-style graphical user interface as well as a syntax-based form, which makes it easy for researchers to specify and estimate models. The macro works with predefined models numbered from model 1 to model 76, all assigned to one special conceptual structure of the focal mediation model and thus enabling it to estimate the most commonly used theoretical model structures ${ }^{10}$. PROCESS requires specification of (i) the model [number] that is to be estimated (a conceptual reasoning that must be clarified a priori and then reflected by the model number one can find in the templates document provided at the PROCESS website) and (ii) the variables included in the model and their associated roles (e. g., independent variable, dependent variable, and mediator). Figure 14 shows the graphical user interface (for SPSS) which can be found under ANALYZE $\rightarrow$ REGRESSION $\rightarrow$ PROCESS after installing the macro (see Hayes, 2013).

\footnotetext{
10 It is recommendable to also download the templates document from the website, which lists all the models
} PROCESS can specify (Hayes, 2017). A full documentation of PROCESS is provided by Hayes (2013). 
Figure 14: Screenshot of the PROCESS Graphical User Interface in IBM SPSS Statistics.

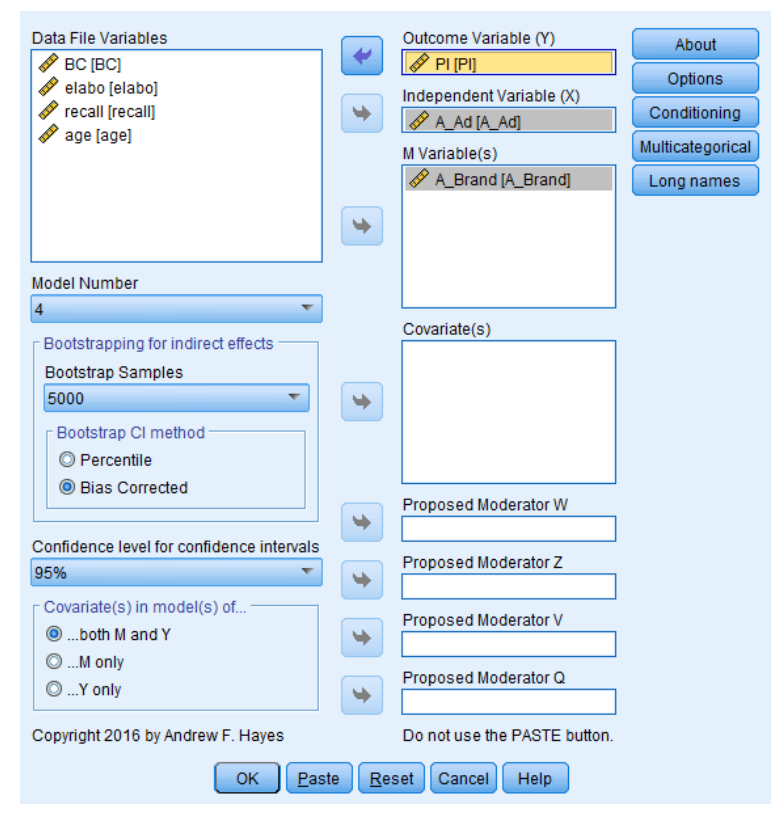

In this section, we illustrate how to specify the examples of the four mediation model groups introduced in Section 2 with hypothetical experimental data ${ }^{11}$. The context of our example is advertising effectiveness, which represents a classic topic in marketing research and provides a suitable framework for testing different forms of mediation models. Specifically, research has suggested processes that are in line with simple mediation (MacKenzie et al., 1986), parallel or serial mediation (S. Brown \& Stayman, 1992), and moderated mediation (MacKenzie \& Spreng, 1992). To facilitate understanding the underlying process, we recommend a hierarchical procedure in which a simple model is considered first, followed by a gradual increase in model complexity. We employ the hierarchical procedure in this tutorial as well and begin with an examination of a simple mediation model, followed by parallel and serial mediation models, and ending with moderated mediation analysis.

\footnotetext{
${ }^{11}$ The data can be downloaded from the Marketing ZFP - Journal of Research and Management website. 
The illustration focuses on the purchase intent of the product advertised $(P I)$ and how it is influenced by attitude toward the ad (hereafter $A_{A d}$; MacKenzie et al., 1986). The example data set also includes variables that are potentially important for the underlying process, namely attitude toward the brand $\left(A_{\text {Brand }}\right)$, product recall (recall), and the elaboration of the ad (elabo). All variables were measured on 7-point Likert scales (with $7=$ "completely agree"), except elabo which was experimentally manipulated (with the levels $1=h i g h$ and $2=l o w$ ). Finally, the data set includes the control variable age. With these variables, we will provide guidance on how to interpret the PROCESS output; suggestions for reporting results for each of the four mediation models are summarized in Appendix 3.

\section{Simple Mediation}

First, we might be interested in answering the question of whether $A_{\text {Brand }}$ mediates the effect of $A_{A d}$ on PI. This corresponds to a simple mediation model, as proposed by MacKenzie et al. (1986) and illustrated in Figure 15.

\section{Figure 15: Simple Mediation Example (PROCESS Model 4).}
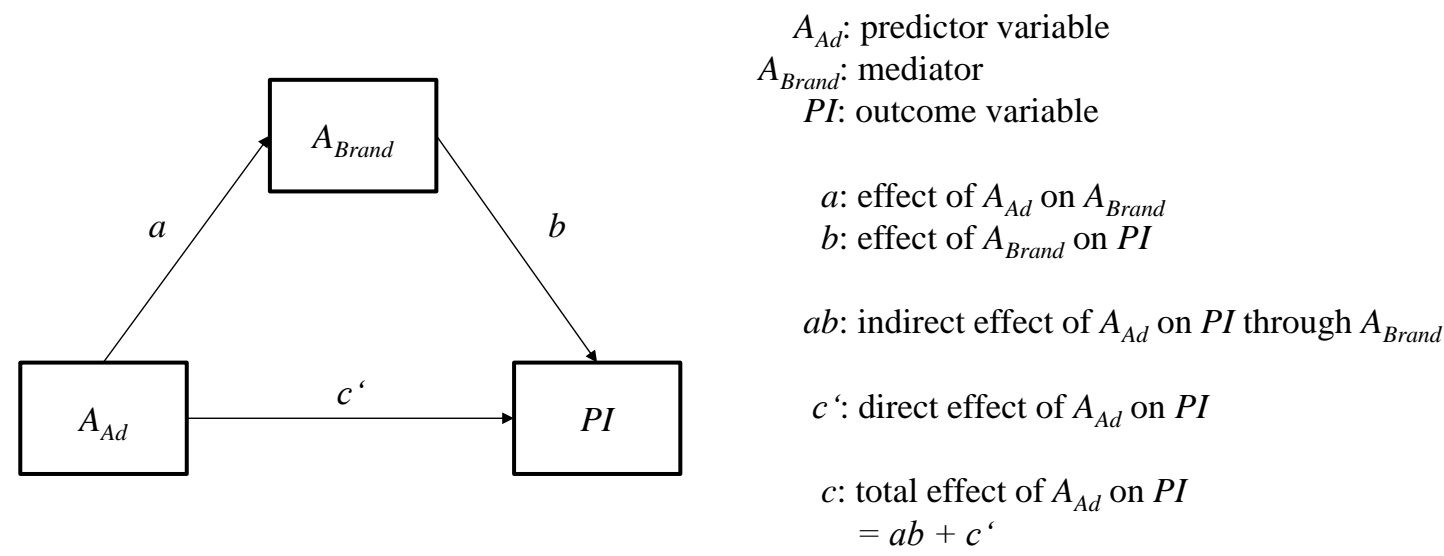


\section{Chapter 4}

Translating the simple mediation model to PROCESS means that we have to choose the model number 4 in the PROCESS template (see Hayes, 2013) and specify the necessary variables. The associated syntax command must be specified as follows ${ }^{12}$ :

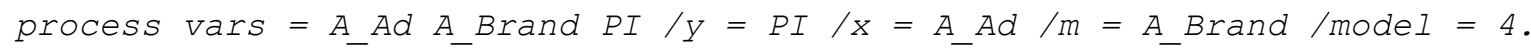

The syntax command first defines that the procedure "process" shall be used. Next, after the "vars $="$ argument, all model variables are listed and assigned to their roles as $X, M$, or $Y$ in the model. The final specification assigns the appropriate model number, " $/$ model $=4 . "$ After running the syntax, PROCESS generates an output, which is divided into different sections, separated by lines of stars (see Figure 16).

\footnotetext{
${ }^{12}$ One can find the specification for this model in the graphical user interface in Figure 14.
} 
Figure 16: PROCESS Output for Simple Mediation Example (Model 4).
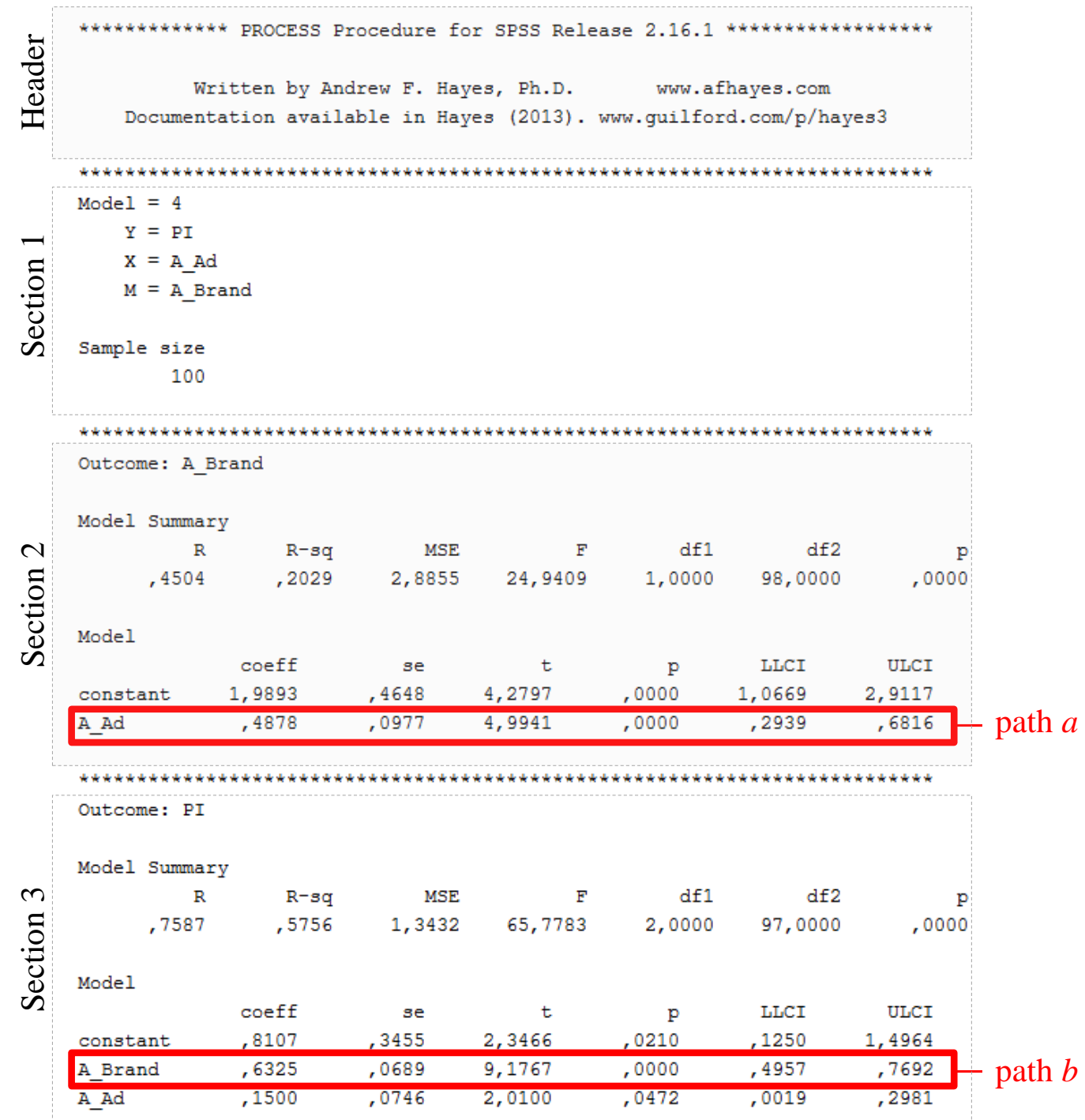

DIRECT AND INDIRECT EFFECTS

Direct effect of $\mathrm{X}$ on $\mathrm{Y}$

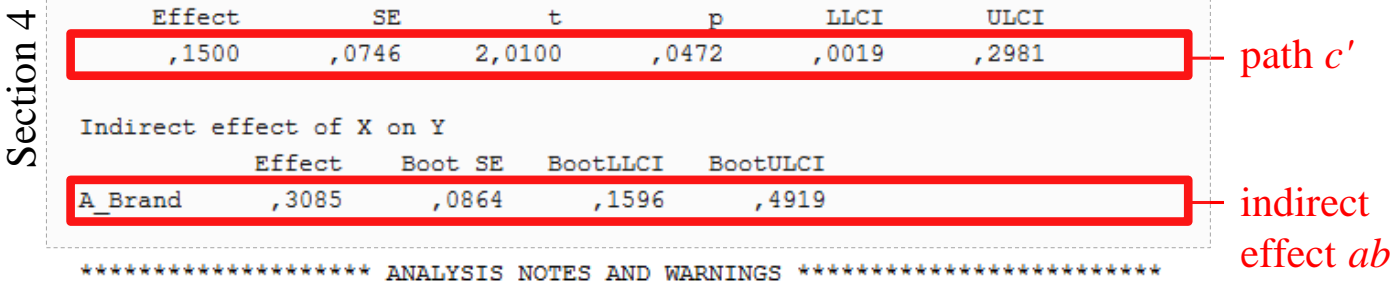

$n$
0
0
0
0
0

Number of bootstrap samples for bias corrected bootstrap confidence intervals: 5000

Level of confidence for all confidence intervals in output: 95,00 


\section{Chapter 4}

Just below the header, the model specification and sample size is presented. The second and third sections plot the simple regression results for paths $a$ and $b$, respectively. The section 4, titled "Direct and indirect effects," is the central part of the mediation analysis output. The last section entails information about the number of bootstrap samples and the level of confidence for all confidence intervals reported in the output.

We can determine whether mediation exists by interpreting the indirect effect of $A_{A d}$ on PI through $A_{\text {Brand }}$ (depicted in the output section 4 as "indirect effect $a b$ "). Using the bootstrap sample distribution explained above (see Section 3.2.), PROCESS reports the point estimate of the indirect effect ("Effect") and its standard error ("Boot SE"). This is followed by the upper and lower limits of the bootstrap confidence interval ("BootLLCI" and "BootULCI"). As can be seen from the output, the bootstrap confidence interval $(C I)$ is (.16 to .49). As the $95 \%$ confidence interval does not include zero, we can infer significant mediation of $A_{A d}$ 's effect on PI through $A_{\text {Brand }}$ at $\alpha=.05$. Next, the importance of the mediation can be assessed by interpreting $A_{A d}$ 's direct effect on $P I$ (depicted as path $c^{\prime}$ ). In the example, the $p$-value of the direct effect is .047 and can therefore be considered significant. Using the framework depicted in Figure 12, we can conclude complementary partial mediation (Zhao et al., 2010). In addition to the direct and indirect effects of the independent variable, PROCESS plots the estimates for the single paths of the model. For the simple mediation model, these can be derived from the results of two regressions underlying the model: one having mediator $A_{\text {Brand }}$ and one having PI as outcome variable. As the indirect effect consists of two single effects, path $A_{A d} \rightarrow A_{B r a n d}$ and path $A_{\text {Brand }} \rightarrow P I$, one can inspect them to reveal that attitude toward the ad impacts purchase intent because it increases attitude toward the brand (effect: .49, $p<.001$; depicted as path $a$ ), while the latter increases purchase intent (effect: .63, $p<.001$; depicted as path $b$ ). Note that the point estimate of the indirect effect $a b$ equals the product of $a \times b: .31=.49 \times .63$. 


\section{Parallel Mediation}

Extending the simple mediation situation, recall could be another potential mediator between $A_{A d}$ and PI (S. Brown \& Stayman, 1992), which would lead to a conceptual model structure like the one shown in Figure 17.

Figure 17: Parallel Mediation Example (PROCESS Model 4).

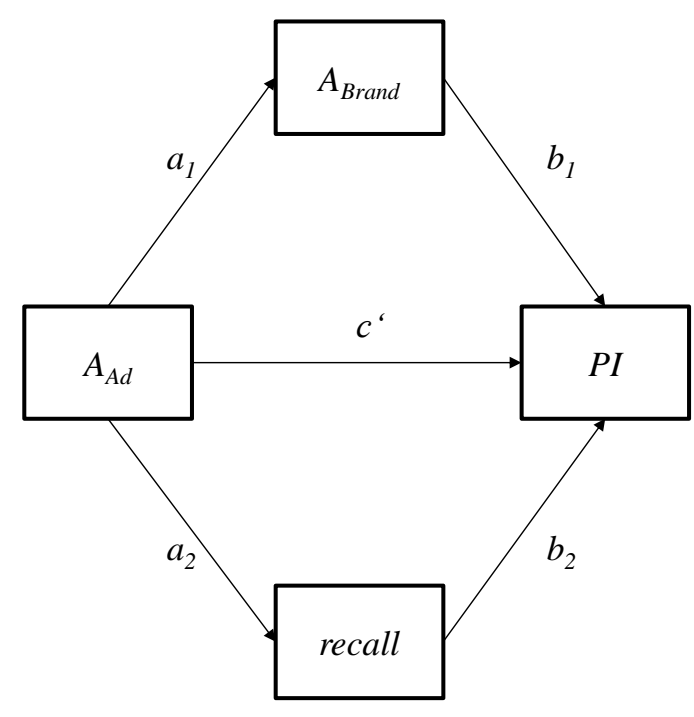

\author{
$A_{A d}:$ predictor variable \\ $A_{\text {Brand }}$ : mediator 1 \\ recall: mediator 2 \\ PI: outcome variable \\ $a_{l} b_{l}$ : specific indirect effect \\ of $A_{A d}$ on $P I$ through $A_{\text {Brand }}$ \\ $a_{2} b_{2}$ : specific indirect effect \\ of $A_{A d}$ on $P I$ through recall \\ $c^{\prime}:$ direct effect of $A_{A d}$ on $P I$ \\ total indirect effect: $a_{1} b_{1}+a_{2} b_{2}$
}

When comparing two proposed mediations it may be of interest to assess whether the corresponding specific indirect effects differ in magnitude. In PROCESS, the "contrast $=1$ " command provides a significance test that can carry out this comparison. If enabled, PROCESS estimates bootstrap confidence intervals for a pairwise comparison of specific indirect effects. It is expressed by a confidence interval because it is based on the bootstrap sampling distributions of both specific indirect effects. If the confidence interval does not entail zero, it implies that the two specific indirect effects are statistically different from each other (if it does include zero, difference between the effects cannot be assumed). It is important to note that this test can only be interpreted as a comparison of effect size when both effects have the same sign. Compared to the simple mediation example, the syntax command is changed in two ways. First, 


\section{Chapter 4}

the variable recall is entered and assigned the role of (second) mediator (" $m=$ recall"). Second, the pairwise comparison option for the specific indirect effects is enabled with "contrast $=1$. ."

process vars $=A \_A d$ A_Brand $P I$ recall $/ y=P I / x=A \_A d / m=A$ B $/$ model $=4 /$ contrast $=1$.

The PROCESS output summary in Figure 18 looks similar to the one-mediator case in Figure 16. However, in this model there are now two specific indirect effects $\left(A_{A d} \rightarrow A_{B r a n d} \rightarrow P I\right.$ and $A_{A d} \rightarrow$ recall $\left.\rightarrow P I\right)$, which together constitute a total indirect effect. For the parallel mediation model, it is most important to interpret these specific indirect effects. In our example, $A_{\text {Brand }}$ (indirect effect: .26; $95 \%$ CI: .14 to .43) as well as recall (indirect effect: .08; $95 \%$ CI: .02 to .17) are significant mediators. If a comparison of the two indirect effects is intended, the contrast bootstrap interval (see line $\mathrm{C} 1$, which means "contrast 1") is examined (depicted as contrast test). In the example, two positive mediations coexist in parallel and differ significantly in size, as the $\mathrm{C} 1$ bootstrap confidence interval does not encompass zero (difference: .17; $95 \%$ CI: .01 to .36). This means that $A_{\text {Brand }}$ can explain the effect of $A_{A d}$ on $P I$ significantly better than recall does. Finally, the total indirect effect should only be interpreted if the researcher wants to investigate the extent to which all mediators together can explain the causal relationship between $A_{A d}$ and $P I$ (depicted as total indirect effect). In the example, the total indirect effect is positive and significant (effect: .34; $95 \%$ CI: 20 to .50). Next, we can interpret the direct effect (depicted as path $c^{\prime}$ ). As the effect is insignificant (effect: .12; $p=$ .075), we can infer full mediation (see Figure 12) ${ }^{13}$. It can also be an option to further investigate the detailed regression results (as in the previous section), but we do not illustrate this here.

\footnotetext{
${ }^{13}$ Some journals, including the Journal of Consumer Research, require reporting $p$-values between .05 and .10 as marginally significant Journal of Consumer Research (2017). Although some researchers argue that marginally significant results should be dismissed Iacobucci (2005), it frequently happens that marginally significant effects are treated as "almost (highly) significant." In the latter case, one would infer complementary partial mediation from the above results.
} 
Figure 18: PROCESS Output Summary of Parallel Mediation Example (Model 4).

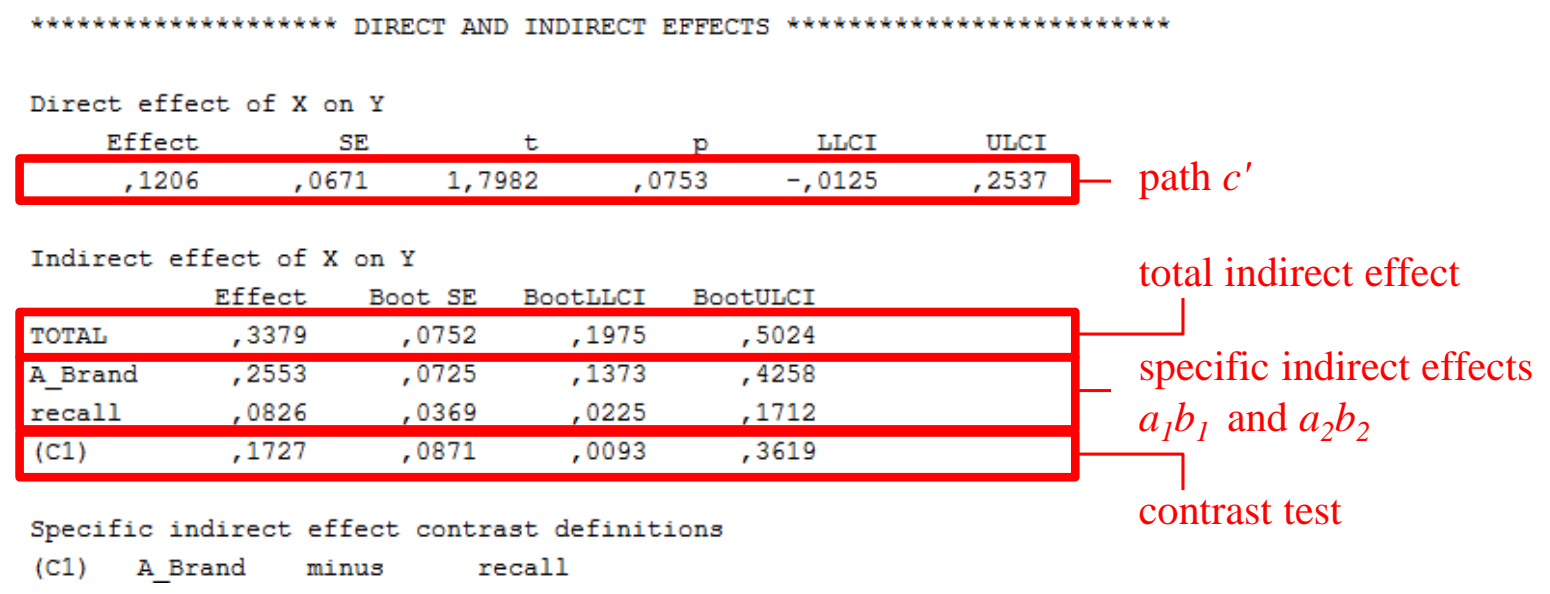

Taken together, the analysis reveals that attitude toward the ad impacts purchase intent through both attitude toward the brand as well as product recall. However, the indirect effect through attitude toward the brand is greater in magnitude than that through product recall. This indicates that attitude toward the brand plays a greater role in explaining the effect of attitude toward the ad than product recall does.

\section{Serial Mediation}

Transferring the perspective of serial mediation to our example, one can also hypothesize that the two mediator variables, $A_{\text {Brand }}$ and recall, are causally related. One plausible assumption could be that $A_{\text {Brand }}$ impacts recall (S. Brown \& Stayman, 1992), which would result in a conceptual model like the one depicted in Figure 19. 
Figure 19: Serial Mediation Example (PROCESS Model 6).
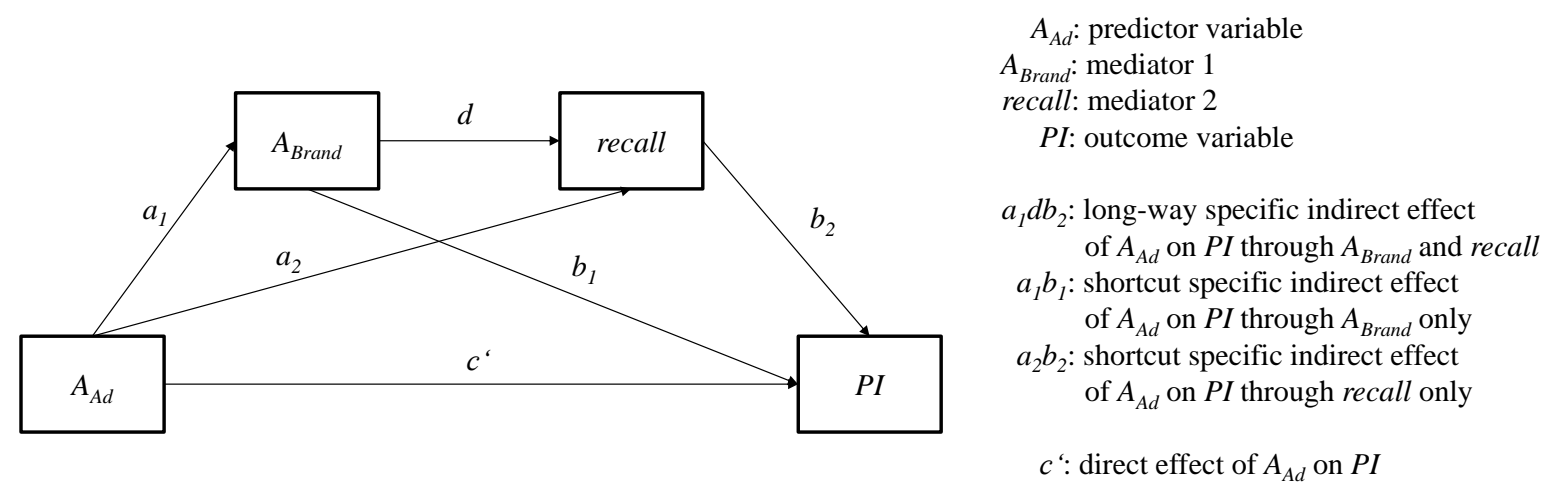

total indirect effect: $a_{1} d b_{2}+a_{1} b_{1}+a_{2} b_{2}$

When specifying serial mediation with model 6 , it is particularly important to consider the order of the mediator variables in the " $m=$ " list, as the variables listed earlier will be regarded causally prior to those listed later. We note that in our example no adjustment of the mediator order is necessary as it already complied with the proposed causal chain. Specifying the framework thus requires two changes to the parallel mediation syntax command: First, the model number must be changed to "model $=6$." Second, the "contrast $=1$ " option is no longer necessary, as the aim of serial mediation is not to compare the mediators. These changes result in the following model specification:

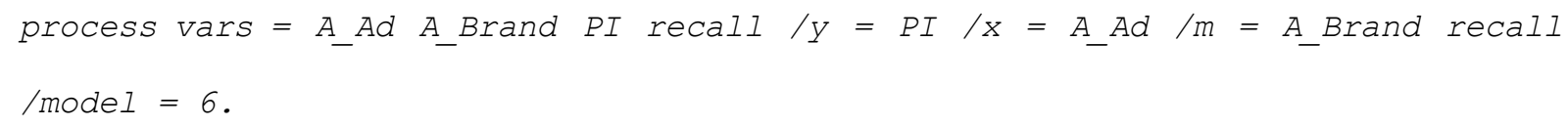

Again, the most important part of the output is the summary section ("Direct and indirect effects"), displayed in Figure 20. PROCESS plots each specific indirect effect as well as the total indirect effect. Central to the serial mediation hypothesis is the long-way mediation $A_{A d} \rightarrow A_{\text {Brand }} \rightarrow$ recall $\rightarrow P I$ (named "Ind2" in the output). If the long-way specific indirect effect is significant, serial mediation can be claimed (depicted as long-way specific indirect effect $a_{1} d b_{2}$ ). Second, we investigate the shortcut indirect effects (depicted as shortcut specific 
indirect effects $a_{1} b_{1}$ and $a_{2} b_{2}$ ) and the direct effect (depicted as path $c^{\prime}$ ) to better understand the character of the proposed mediation paths. As before, it can also be an option to further investigate the detailed regression results (which are not illustrated here).

Figure 20: PROCESS Output Summary of Serial Mediation Example (Model 6).

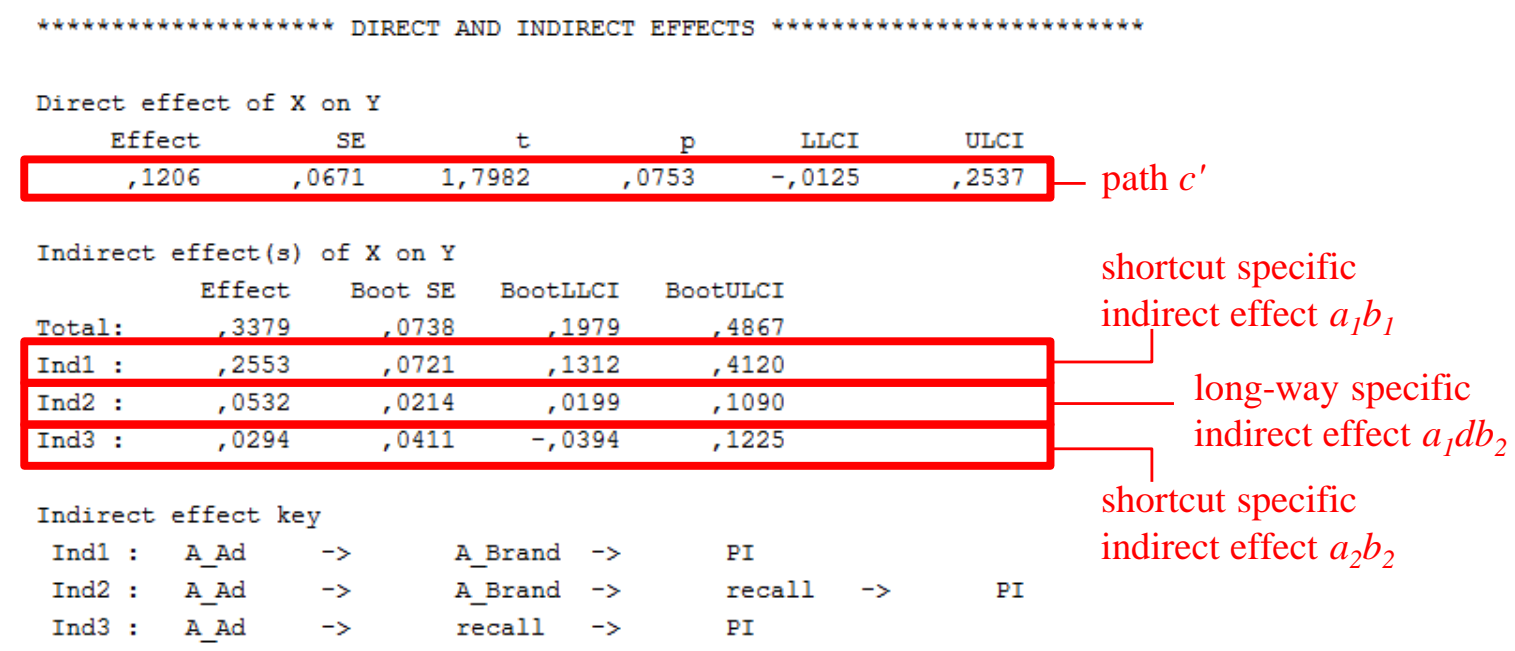

The output of the example in Figure 20 suggests that the long-way mediation $A_{A d} \rightarrow A_{\text {Brand }} \rightarrow$ recall $\rightarrow P I$ is significant (effect: .05; $95 \%$ CI: .02 to .11), as is the indirect path $A_{A d} \rightarrow A_{\text {Brand }} \rightarrow P I$ (effect: $.25 ; 95 \%$ CI: .13 to .41 ). In contrast, the indirect effect of $A_{A d} \rightarrow$ recall $\rightarrow P I$ is not significant (95\% CI: -.04 to .12). Like in the parallel mediation example, the direct effect is insignificant $(p=.075)$. Taken together, the data support the serial mediation hypothesis: attitude toward the ad increases attitude toward the brand, which in turn increases product recall, which ultimately affects purchase intent. In addition, attitude toward the ad affects purchase intent via attitude toward the brand, without product recall being involved. This finding qualifies the result of the parallel mediation example: product recall does not act as an independent mediator but rather is part of a longer causal chain that involves attitude toward the brand. 


\section{Chapter 4}

\section{Moderated Mediation}

Extending the "pure mediation" example, one could imagine that the test person's processing elaboration of the ad (elabo) might be a variable that determines whether the proposed mediation process $A_{A d} \rightarrow A_{B r a n d} \rightarrow P I$ exists. Specifically, it is conceivable that in the case of low elaboration the proposed mediation works, while $A_{A d}$ does not lead to an increase in $A_{B r a n d}$ in case of high elaboration (MacKenzie and Spreng 1992). In this case a first-stage moderated mediation model in PROCESS is suitable, as shown in its most basic form (model 7) in Figure 21.

Figure 21: Moderated Mediation Example (PROCESS Model 7).

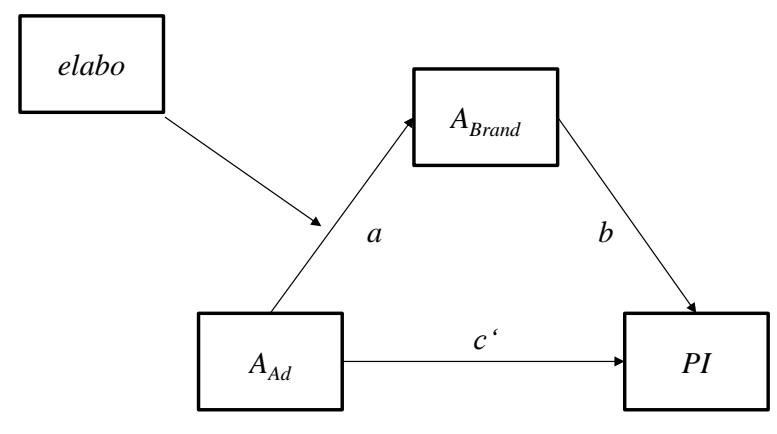

$$
\begin{aligned}
& A_{A d}: \text { predictor variable } \\
& A_{B r a n d}: \text { mediator } \\
& P I \text { : outcome variable } \\
& \text { elabo: moderator } \\
& a b \text { : indirect effect of } A_{A d} \text { on } P I \text { through } A_{B r a n d} \\
& \quad c^{\prime}: \text { direct effect of } A_{A d} \text { on } P I \\
& \text { conditional indirect effect: } \\
& \text { indirect effect } a b \text {, conditional on levels of elabo } \\
& \text { being high (1) or low (2) }
\end{aligned}
$$

To specify such a model in PROCESS, one must adjust the syntax command of the simple mediation model to include the variable elabo and assign it the moderator role with " $/ w$ $=$ elabo." Moreover, the model number must be changed to "model $=7 . "$

process vars $=A \_A d$ A_Brand $P I$ elabo $/ y=P I / x=A \_A d / m=A \_B r a n d$ $/$ model $=7 / \mathrm{w}=$ elabo

The output shown in Figure 22 provides a summary of the direct and indirect effects. The indirect effect is now conditional on the values of the moderator (the subsection is therefore called "Conditional indirect effect( $(s)$ of $X$ on $Y$ at values of the moderator( $(s)$ "). 
Figure 22: PROCESS Output Summary of Moderated Mediation Example (Model 7).

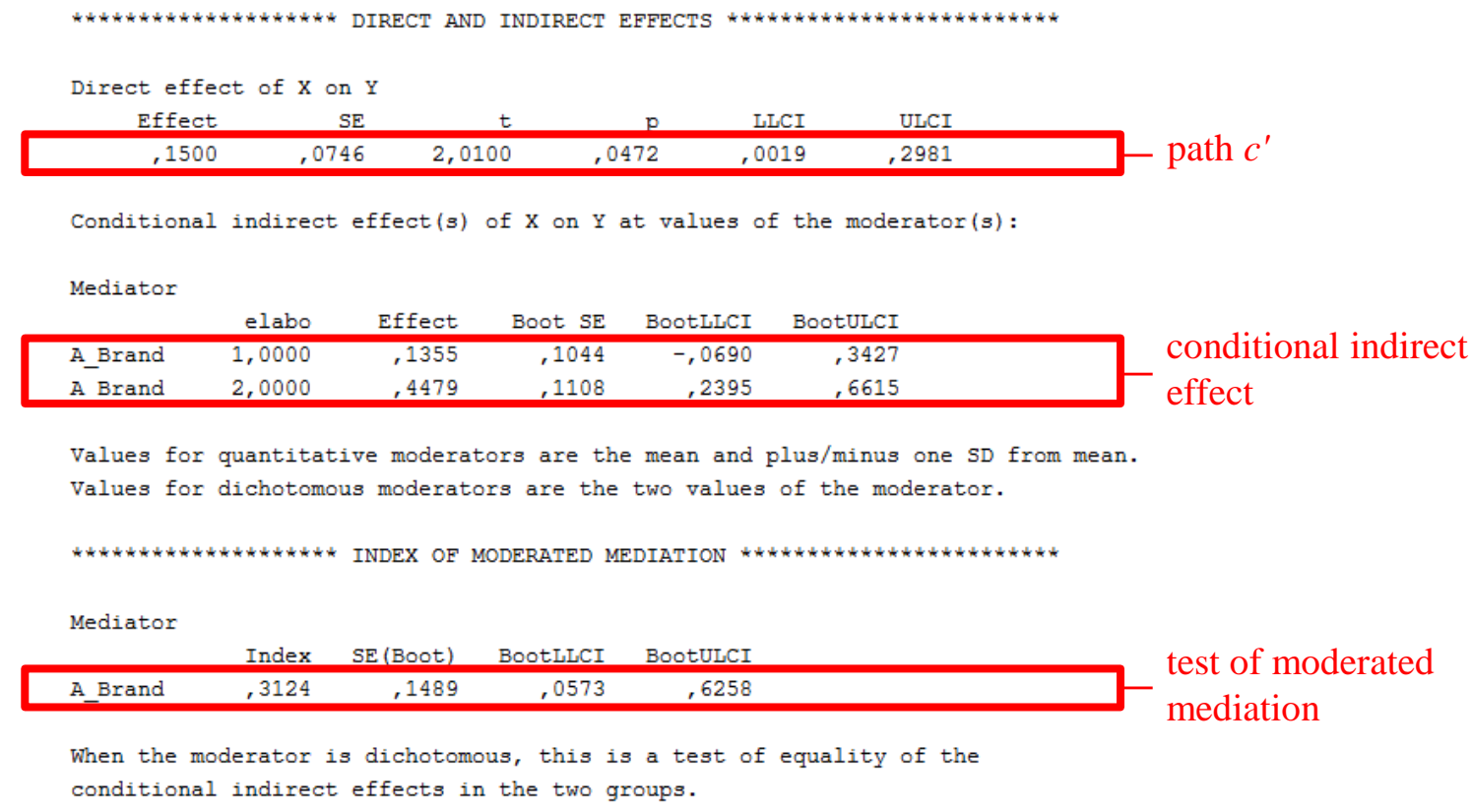

To interpret the output, it is necessary to examine whether the proposed moderated mediation exists. This can be achieved by carrying out a formal test of moderated mediation called the index of moderated mediation (Hayes, 2015), depicted as such in Figure 22. The index represents the quantification of the linear association between the moderator and the indirect effect. Like before, it is a bootstrap confidence interval that is interpreted as support for the existence of moderated mediation if it does not include zero. As here the confidence interval does not include zero (95\% CI: .06 to .63), the hypothesis of moderated mediation is supported. This means that the indirect effect of $A \_A d$ on $P I$ through $A \_B r a n d$ depends on levels of elabo.

If the index of moderated mediation supports the existence of moderated mediation, one may wish to investigate the indirect effect at representative values of the moderator (depicted as conditional indirect effect) to further explore the conditions under which mediation does (not) exist (Preacher et al. 2007). This method is also called spotlight analysis (Fitzsimons, 


\section{Chapter 4}

2008; Spiller, Fitzsimons, Lynch, \& McClelland, 2013). If the moderator is dichotomous, it results in exactly two conditional indirect effects. For a continuous moderator, by default the conditional indirect effects for the moderator mean and at values of one standard deviation above and below the mean are plotted. As in the examples before, the mediation type can be determined by additionally considering the direct effect (depicted as path $c^{\prime}$ ). In the example we see that while there is a significant indirect effect for test persons with low elaboration (effect: $.45 ; 95 \%$ CI: .24 to .66), the effect is not significant in the high-elaboration group (95\% CI: -.07 to .34). The direct effect is significant ( $p=.047)$, implying partial mediation. From a theory perspective, we can hence conclude that attitude toward the ad affects purchase intent via attitude toward the brand only when the ad is not elaborated deeply.

\section{Further Modifications of the Mediation Analysis}

Besides the extensions discussed, PROCESS allows further syntax statements to modify the analysis according to the researcher's goals. In Table 5 we describe other potential needs for modification of the default syntax command and how to address them. 
Table 5: Additional Statements of the PROCESS Syntax.

\begin{tabular}{|c|c|c|}
\hline Goal & Description & Syntax Statement \\
\hline $\begin{array}{l}\text { Control for the influence of other variables in } \\
\text { the model by including covariates }\end{array}$ & $\begin{array}{l}\text { Listing variables after "process vars =" without } \\
\text { assigning roles makes PROCESS treat these variables } \\
\text { as covariates. }\end{array}$ & $\begin{array}{l}\text { "process vars = } \\
\text { [additionally include } \\
\text { name of covariate]" }\end{array}$ \\
\hline $\begin{array}{l}\text { Make bootstrap results reproducible and } \\
\text { avoid variances in results due to resampling } \\
\text { when repeating the same analysis }\end{array}$ & $\begin{array}{l}\text { PROCESS output values always differ slightly due to } \\
\text { the random resampling process. The statement is } \\
\text { seeding the random number generator responsible for } \\
\text { resampling with an arbitrary value, which leads to } \\
\text { exactly reproducible results. }\end{array}$ & $"$ "/seed $=$ [number]" \\
\hline $\begin{array}{l}\text { Make the estimation of confidence intervals } \\
\text { more precise }\end{array}$ & $\begin{array}{l}5,000 \text { bootstrap resamples is the default in } \\
\text { PROCESS. The higher the number of bootstraps, the } \\
\text { more reliable the results become. } 10,000 \text { bootstraps } \\
\text { are a good compromise between desired precision and } \\
\text { required computation time. }\end{array}$ & "boot = [number]" \\
\hline $\begin{array}{l}\text { Change the bootstrapping approach from the } \\
\text { default bias-correct bootstrap to percentile } \\
\text { bootstrapping }\end{array}$ & $\begin{array}{l}\text { Percentile bootstrapping is especially robust in small } \\
\text { samples or adverse situations. }\end{array}$ & $"$ percent $=1 "$ \\
\hline $\begin{array}{l}\text { Save the estimated bootstraps of all paths of } \\
\text { the model in a new SPSS data set to compare } \\
\text { confidence intervals of parameters, for } \\
\text { example. }\end{array}$ & $\begin{array}{l}\text { Saving the bootstraps makes it possible to manually } \\
\text { compare paths or function of paths (e. g., the indirect } \\
\text { effect is a function of a and b). In the new data set, } \\
\text { each row contains the coefficients from one bootstrap } \\
\text { sample (e. g., } \mathrm{i}_{1}, \mathrm{a}, \mathrm{i}_{2}, \mathrm{~b}, \mathrm{c}^{\prime} \text { for the simple mediation } \\
\text { model named COL1 to COL5). }\end{array}$ & $"$ save $=1 "$ \\
\hline $\begin{array}{l}\text { Perform mediation analysis with a } \\
\text { multicategorical independent variable (e. g., } \\
\text { two experimental conditions plus a control } \\
\text { group coded " } 1=\text { control," " } 2=\text { treatment } \\
\text { A," and " } 3 \text { = treatment B") }\end{array}$ & $\begin{array}{l}\text { Unlike continuous or dichotomous variables, } \\
\text { multicategorical independent variables cannot just be } \\
\text { included in mediation analysis without } \\
\text { transformation (categorical independent variables } \\
\text { with more than two levels are interpreted as linear, } \\
\text { which leads to biased parameter estimates). } \\
\text { PROCESS is able to recode such a multicategorical } \\
\text { variable automatically via the command "/mcx =", } \\
\text { with "/mcx = 1" being the flag for dummy coding (for } \\
\text { a detailed description of the analysis, see Hayes \& } \\
\text { Preacher, 2014). Note that multicategorical } \\
\text { independent variables are currently possible in simple } \\
\text { mediation models only. }\end{array}$ & $" / m c x=1 "$ \\
\hline
\end{tabular}




\section{Chapter 4}

The following syntax command shows an example application of these extensions based on the simple mediation syntax command for the control variable age, a seed starting the random number generator at position 100, and 10,000 bootstrap resamples using the percentile bootstrapping approach and a multicategorical independent variable:

process vars $=A \_A d$ A_Brand $P I$ age $/ y=P I / x=A \_A d / m=A \_B r a n d / m o d e I=4$

$/$ seed $=100 /$ boot $=10000 /$ percent $=1 /$ save $=1 / \mathrm{mcx}=1$.

\subsection{Robustness Assessment and Complex Model Testing}

In the last section, we examine two topics worth considering for a deeper understanding of how to conduct mediation analysis. Although there have been many advanced topics discussed in the recent literature, such as how to handle longitudinal data (Preacher, 2015) or analyzing nonlinear effects (Hayes \& Preacher, 2010), we limit the scope of this section to two basic topics: (i) the importance of making robust causal inference via correct specification and (ii) the use of structural equation modeling as an alternative for analyzing complex mediation models.

\subsubsection{Assessing the Robustness of the Causal Inference}

As discussed in the context of partial mediation, a rigorous specification of the proposed model is of utmost importance in order to identify genuine mediation processes. We also examined the omitting of alternative mediators or moderators as examples of incomplete mediation findings. However, there are further misspecifications that could turn a genuine full mediation into a result of partial mediation or insignificance, such as non-linearity of causal relationships, measurement error, missing paths, or outliers (Creedon \& Hayes, 2015). To overcome these potential sources of misspecification, the standard means known from regression analysis should be applied (e. g., visual inspection, outlier detection, inspection of residuals). 
Even in the case of a significant full mediation, the researcher should reflect upon the specification of the proposed model to ensure that the results are meaningful. If this is not done carefully, what has been identified as a mediator in the proposed model might in reality play a different causal role (MacKinnon, Krull, \& Lockwood, 2000; Stelzl, 1986). For example, the proposed mediator $M$ may not be a real causal mediator, but rather just a correlate of $X, Y$, or the true mediator that is not specified in the model at all. Therefore, inference about the proposed mediation cannot be based on a significant indirect effect alone; instead, significance of the mediation is just a necessary condition following a-priori conceptual reasoning (Fiedler, Schott, \& Meiser, 2011). It is therefore important to make sure that one can conceptually and empirically justify the proposed model against rival explanations (Iacobucci et al., 2007). In this regard, it makes sense to test other possible model specifications and compare them to the focal model (and report the results accordingly). If one can rule out the potential sources of misspecification discussed above, this enhances confidence in the proposed theoretical framework.

It is important to note that there are no formal means of examining the fit of a model (like the multiple fit indices known from SEM; see Section 5.2.) when using PROCESS. In situations where more than one model is both conceptually and empirically meaningful (e. g., significant and interpretable indirect effects in parallel and serial mediation analysis), we follow Hayes (2013) and recommend preferring those models which are less restricted in their assumptions. In the given example, this would mean preferring the serial model over the parallel model, as it allows both mediators to be connected, while the parallel model implicitly assumes that the mediators are unrelated (i. e., a correlation of exactly zero). 


\section{Chapter 4}

\subsubsection{Testing Complex Mediation Models with SEM}

In this paper we have illustrated how to use regression-based mediation analysis via PROCESS for moderately complex mediation models. This approach is sufficient for most research settings, as simpler models with fewer variables are generally preferable to more complex ones (J. Cohen, 1990). However, there might be situations that require complex mediation models that cannot be analyzed with PROCESS. For example, a requested model may contain more than one independent or dependent variable.

SEM offers a methodology for analyzing causal relationships between multiple latent variables (Bollen, 1989; Iacobucci, 2010). Hence, SEM allows the examination of complex nomological networks (Iacobucci, 2008), such as serial mediation models with multiple dependent and independent (in SEM terminology: exogenous) variables. In addition to the number of structural relationships, SEM is also flexible regarding the type of relationships. For example, it is possible to model non-recursive relations of the form $\mathrm{Y} 1 \longleftrightarrow \longrightarrow \mathrm{Y} 2$ (Iacobucci, 2009).

Another advantage of SEM is that it explicitly considers latent variables with multiple indicators that are measured with error, whereas in regression-based research multi-item measurements of variables are typically collapsed to mean scores (Iacobucci, 2009). If the items measure the latent construct inconsistently (i. e., factor loadings are not uniformly high), this simplification can reduce the likelihood of finding systematic relationships in regression-based mediation analysis (Danner, Hagemann, \& Fiedler, 2015). Iacobucci et al. (2007) demonstrate that inferring mediation from mean scores for $X, M$, and $Y$ may lead to misleading results compared to full measurement models, especially when the mediation effect is small. In conclusion, it may be beneficial to consider measurement error if the measurement of variables is problematic (which can be identified via factor analysis or reliability analysis). 
A third advantage of SEM is its provision of model fit indices (Hu \& Bentler, 1998; Iacobucci, 2010). Fit indices can be used to assess the adequacy of a proposed model, but they also enable the comparison of different models. Formal comparison is useful because sometimes a researcher is unsure which theoretical model is most promising. For instance, the so-called $\chi^{2}$ difference test is able to test whether two nested models significantly differ; the better fitting model is indicated by the smaller $\chi^{2}$ value (for details on model comparison, see Danner et al., 2015). Furthermore, SEM allows one to constrain paths inside the structural model if there is theoretical reason to do so (e. g., to set them to zero or to set several paths to the same value). Such constrained models can also be compared to more parsimonious alternatives via fit indices that take into account the parsimony of the models (e. g., Bayesian Information Criterion; see Danner et al., 2015).

In the case of models that cannot be specified with PROCESS, using established SEM software like AMOS (Arbuckle, 2016) or Mplus (Muthen \& Muthen, 1998-2015) appears to be a good choice. Such SEM programs offer bootstrapping as an estimation method (Cheung \& Lau, 2008), which we also recommend using in SEM because of the non-normal nature of the indirect effect. If bootstrapping is applied, SEM yields nearly the same results as PROCESS (for the four examples, the results in AMOS differ from the ones in PROCESS only on the third decimal). Despite the opportunities SEM presents for specifying mediation models, it also means more complexity in setup and analysis - for example, when comparing alternative models in the presence of contradicting fit indices (Iacobucci et al., 2007).

Furthermore, most of the SEM programs do not offer the full functionality of PROCESS regarding mediation analysis. For instance, while it is possible to test the total indirect effect in most of the SEM programs, parameters and confidence intervals of specific indirect effects in mediation models are usually not part of the output (exceptions are Mplus, Mx, and OpenMx; 


\section{Chapter 4}

Macho \& Ledermann, 2011). Nonetheless, it is possible to manually implement some of the features. For example, there exists a methodology to estimate specific indirect effects in SEM programs such as AMOS, called the phantom model approach (Macho \& Ledermann, 2011). Macho and Ledermann's idea for estimating a specific indirect effect is to build a separate partial model (phantom model) that mimics the full model but only encompasses the paths of the specific effect (for detailed instruction on how to build a phantom model, see Macho \& Ledermann, 2011). Other examples in which SEM programs do not provide as much default features as PROCESS is in the interpretation of moderated mediation (e. g., they do not yet provide an index of moderated mediation) or mediation models with multicategorical independent variables (the so-called omnibus test; Hayes \& Preacher, 2014). Researchers interested in these specific features in complex model settings may prefer Mplus. The syntaxbased nature of Mplus makes it possible to access code concerning such advanced mediation analysis features (e. g., code for complex moderated mediation models in Hayes \& Preacher, 2014).

In summary, SEM is a powerful approach that can enhance mediation analysis in several ways. However, one should keep in mind that its sophistication poses several challenges and therefore inexperienced researchers might face some error potential when using it. A decision rule would be that regression-based bootstrap approaches (such as those offered by PROCESS) are preferable unless the issues described in this section become crucial.

\subsection{Conclusion}

The goal of this paper was to give an overview of mediation analysis. To achieve this goal, we reviewed the basic concept of mediation as well as its main elements, and we discussed how to interpret mediation results based on indirect and direct effects. We focused on simple mediation, parallel mediation, serial mediation, and moderated mediation, which represent the 
most common mediation model groups in marketing research. After reviewing the conceptual background of mediation analysis, we turned our attention to the methodological aspects of mediation analysis. Here, we compared three different regression-based approaches of mediation analysis. In particular, we examined the rationale of the bootstrapping approach, discussed why it yields superior results compared to traditional approaches of mediation analysis, and argued that it is particularly suitable for estimating the indirect effect. While we concentrated on conceptual and methodological considerations in the first part of the paper, the second part is organized as a tutorial. Here, we illustrated how to conduct mediation analysis and interpret the output of the SPSS/SAS macro PROCESS.

In the tutorial, we presented a typical case of more than one model specification being theoretically meaningful and recommended a hierarchical procedure. Hereby one examines simple mediation models first and, step by step, extends those simple models to more complex models. Following such a stepwise approach, we opted for inspecting the indirect effect at each step to determine whether a proposed mediator can explain the proposed causal relationship. Moreover, we recommended inspecting the direct effect to reveal to what extent the causal relationship can be explained by the mediator. This information is useful for further conceptual reasoning, and we describe a suitable framework in Section 2.3. Besides inspecting the direct and indirect effect in simple and parallel mediation, we also illustrated how to test more sophisticated hypotheses of a serial mediation model and a moderated mediation model. For the serial mediation model, we emphasized the significance of the long-way specific indirect effect as most important. For the moderated mediation model, we recommended to test whether the proposed indirect effect is conditional on levels of a moderator, revealed by the index of moderated mediation. After giving detailed information on how to specify the models with PROCESS syntax and interpret the relevant elements of the PROCESS output, in Appendix 3 


\section{Chapter 4}

we illustrated how to report results. Concluding, we hope that this review and tutorial will contribute to a consistent and cognizant use of mediation analysis. 


\section{General Conclusions}

Recommendations effectively influence product judgments and purchase decisions. However, as consumers nowadays are faced with many recommendations, they often have to choose which recommenders they follow. Previous research has examined whether performing good or bad in prior recommendation leads to changes in intent to follow a recommender, and under which conditions performance information is especially considered. Specifically, it has been shown that consumers utilize performance information when it is presented in an aggregated form (Gershoff et al., 2001), and that negative performance information, i.e., failed recommendations, especially count (Yaniv \& Kleinberger, 2000). Prior research has also yielded valuable insights for the question how other characteristics like expertise or similarity of recommenders shape the evaluation of recommenders and, consequently, promote intentions to follow (Tuk, Verlegh, Smidts, \& Wigboldus, 2019; e.g., White, 2005). Perhaps the best researched characteristic in recommendation literature is the relationship closeness between recommender and recommendation receiver (e.g., Brown \& Reingen, 1987; Granovetter, 1973).

However, prior research has so far largely neglected the interplay of recommendation performance and relationship closeness and instead separately examined its effects. This may have led to an incomplete picture suggesting that both cues work independently. Thus, research that examines how recommendation receivers respond towards recommendation performance when this information comes from close others is lacking. The starting point for this dissertation was the attempt to understand how consumers process recommendation performance information when recommenders have strong ties and how this information processing differs from those of recommendations of distant others. We were especially interested how consumers respond to unexpectedly bad recommendation performances of close others. 


\section{Chapter 5}

\subsection{Research Implications}

Our work advances the existing paradigm of recommender evaluation by providing extensive insights for research and practice regarding the questions of whether, how and when the response to recommendation performance of close others differs from that of distant others. First and foremost, this work provides clear evidence that relationships matter when recommendation receivers evaluate performances of recommenders. However, our results from Paper 1 suggest that there is no general favorability of close others in terms of recommender evaluation. On the one hand, this means that recommendation performance of close others is not interpreted more favorably. This finding rules out the claim that a biased interpretation of recommendation outcomes may be responsible for the overestimated belief in close others' ability to recommend as shown in other contexts. For example, research has demonstrated that mechanism of biased interpretation in order to stick with existing beliefs may bias the evaluation of politicians (Slothuus \& Vreese, 2010) and that relational bonds can lead to more favorable interpretations of a person's traits (Boucher, 2013). In contrast to other contexts, our findings suggest that consumers do not substantially "overvalue" recommendation performances of close others. This result seems to suggests that the unbiased interpretation of performance is simply used in a more favorable way for the evaluation of close recommenders. However, we established that recommendation performance information is not always utilized in a way that favors the evaluation of close others. In contrast, good recommendation performance appears to increase the influence of close and distant recommenders in a similar way, while only bad recommendation performance information for close others is utilized less. We refer to it as the asymmetric effect of relationship closeness on performance utilization. This finding is in line with an asymmetric effect Gerhoff and Johar (2006) found with regard to the calibration of personal knowledge of close and distant others. Interestingly, the observed 
asymmetric effect of relationship closeness also attenuated the general finding that recommender evaluation underlies a negativity bias (Yaniv \& Kleinberger, 2000). We showed that individuals do not always perceive negative performance as more meaningful than positive performance. Instead, they weigh information about good and bad prior performance of close others equally high. Although such a strategy is weak in its ability to avoid unsuitable recommenders, it might not always be bad. For example, it gives a second chance to recommenders with high abilities who have performed poorly before for some reason.

One major finding from Paper 2 is that not only recommenders but also companies can benefit from relationship closeness to recommenders in the case of negative recommendation outcomes. We showed that the acquisition by recommendations of close others protects firms from negative consequences in case of service failure in the first encounter. Further, we established that this effect is driven by a shift in attribution about the failure. This is remarkable as it means that favorable reasoning about the recommender appears to spill over to the causal reasoning about a service failure caused by a firm. Consequently, our findings complement research that has established that close firm-customer relationships act as a buffer that may alter how consumers reason about occurring failures (Hess et al., 2003).

Across both two empirical papers, besides the focal effect of relationship closeness we also analyzed the effect of recency (recent vs. less recent recommendation performance) and examined potential underlying psychological mechanisms (interpretation of performance information and attribution of negative outcome). The results of the papers show the relevance of these important factors to explain our findings.

Generally, the examination of response to bad recommendations of friends, in addition to the previously studied general performance information processing, is a valuable extension of 


\section{Chapter 5}

extant research and adds to an evolving body of literature that studies the role of relationships on consumer decision making (Canavaugh 2016). Our research contributes to the conversation on how consumers use performance and relational information that are not consistent. We establish that relationship closeness not only acts as a cue that enhances recommender influence but also inhibits utilization of performance information when it is inconsistent with valued beliefs.

Lastly, Paper 3 provides an overview of what mediation analysis means, which approaches exist to establish mediation, and how to conduct mediation analysis with state-of-the-art methodology. The detailed methodological discussion of mediation analysis in Paper 3 should help researchers to plan, execute, and analyze research that includes complex mediation relationships.

\subsection{Managerial Implications}

Our research may also provide guidance to managers. For example, company representatives often act as advisors to consumers. Paper 1 suggests that deep relationships with clients can buffer response to recent advice that was suboptimal and may give company representatives a second chance to advise. When companies have built close relationships with customers they could even advocate controversial options without instantly losing their influence. Another finding is that a recent successful recommendation substantially enhances recommender influence independent from relationship closeness. For example, companies could use an initial recommendation in an irrelevant matter with a new client to increase the acceptance rate for a subsequent focal recommendation.

Paper 1 also offers managerial implications for companies that utilize WOM marketing. Our findings suggest that targeting friends for seeding strategies is a sustainable strategy, as 
they do not loose influence quickly even when they provide bad advice. Also, we find that distant recommenders who have performed well recently are nearly as influential as close recommenders. These recommenders have not yet been targeted by seeding campaigns but offer high potential.

In Paper 2 we suggest that retention of other customers after service failure is an additional value driver of customers that have recommended close others. These close relationship recommenders seem to have a special importance for companies beyond only acquiring new customers. In addition, new customers appear to be more forgiving towards failure as a consequence of recommendations by close others. Companies should consider the protective effect of close recommenders as an additional component of a customer's engagement value (Kumar et al., 2010). Finally, our findings underline the importance of encouraging existing customers to generate company-related WOM especially to strong-ties, for example by tell-a-friend campaigns.

Overall, the results of the dissertation help researchers to understand individual processes on the effect of bad recommendations from close others and provide managers guidelines to implement recommendation marketing strategies that consider our findings. Our results suggest that consumers process bad recommendation performance from close others substantially different than those of distant others, whilst no difference was evident for positive performance. Finally, we show how companies can benefit from our findings. While our research does not necessarily suggest that all recommendation marketing efforts should be targeted at close recommenders, we provide novel insights that extend previous perspectives about the suitability of close others as recommenders. 
This page is intentionally left blank. 


\section{Appendix}

\section{Appendix 1A: Scenarios, Manipulations and Phrasing of Questions in Paper 1, Main Study}

Introduction. In this study, you will be presented a recommendation scenario for movies. We kindly ask you to read the scenario and really try to imagine yourself as the consumer. In other words, please respond just as you would if you were actually experiencing the described situation:

Imagine that you want to go to the cinema on the weekend, but still have no idea which movie you want to see.

\section{Manipulation of Relationship Closeness.}

(Acquaintance Condition)

Please think of an acquaintance (someone who is not as close to you as a friend), who you could imagine recommending you a movie.

It is especially important that you think of a real person you actually know.

Take a few moments to find a suitable person.

\section{(Friend Condition)}

Please think of a close friend (someone who is closer to you than an acquaintance), who you could imagine recommending you a movie.

It is especially important that you think of a real person you actually know.

Take a few moments to find a suitable person.

Recommender Name. In the following scenario, we will refer several times to the person you just thought of. Please enter the first name of this person in the input field below. (text field)

Scenario of First Movie Recommendation. Imagine, you are telling [name] that you intend to go to the cinema on the weekend but do not have any idea what movie you would like to see. [name] recommends you a certain movie with the words:

"You should watch this movie, it's just the way you like movies!"

\section{Covariate.}

Initial Intent to Follow Recommender. When assuming that [name] would not know if you actually chose the recommended movie or not:

How likely would you follow the recommendation and choose the movie that [name] just recommended to you? (7-point scale, screenshot of computer interface below)

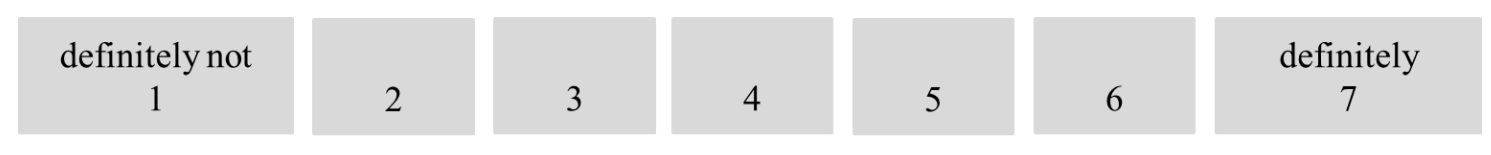




\section{Appendix 1A: Scenarios, Manipulations and Phrasing of Questions in Paper 1, Main Study (cont.)}

\section{Manipulation of Recommendation Performance. \\ (Good Performance Condition $)^{14}$}

Please imagine that you actually watched the movie recommended by [name].

When you watch the movie at the cinema, you experience it like this:

The plot develops and has many turns. There are medium-fast scene changes, but overall the pace is not hectic. The plot focuses on a manageable number of protagonists whose dialogues are concise. The movie ends in a way you did not expect it would from the start. The movie lasts about two hours.

\section{(Bad Performance Condition)}

Please imagine that you actually watched the movie recommended by [name].

When you watch the movie in the cinema, you experience it like this:

The plot unfolds more in the background and contains hardly any twists. There are few scene changes, the overall pace is rather slow. Many characters appear in the movie and extensive dialogues dominate. The movie ends as you expected it would from the start. The movie lasts about three hours.

Performance Evaluation. (3 items, 7-point scales, anchors below)

How well does this movie meet your taste? (not at all --- very much)

How would you rate the quality of this movie? (very low --- very high)

How would you rate the recommendation of [name]? (very unsuitable --- very suitable)

Scenario of Subsequent Movie Recommendation. A few weeks later you want to go to the cinema once more, again without having a specific movie in mind. When you tell [name] about it, [name] again recommends you a certain movie with the words:

"You should watch this movie; it really meets your preferences!"

Main Dependent Variable.

Intent to Follow Recommender. When assuming that [name] again would not get to know if you actually chose the recommended movie or not:

How likely would you follow the recommendation and choose the movie that [name] just recommended? (7-point scale, screenshot of computer interface below)

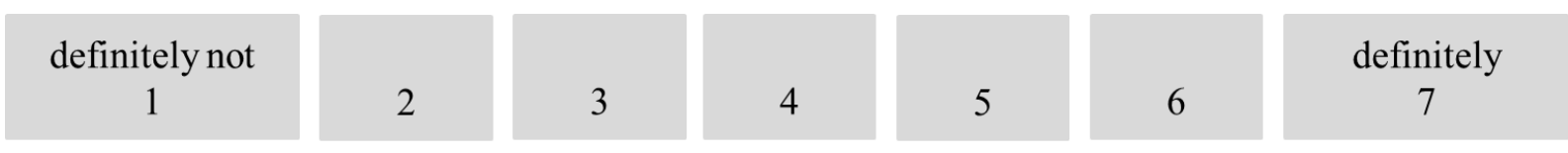

\footnotetext{
${ }^{14} \mathrm{We}$ chose these vignettes from a pre-test. In this pre-test we present six potential vignettes in a randomized order to 53 students from a marketing course for partial course credit. The participants of the pre-test evaluated each of the vignettes on a 7-point scale. We created the six vignettes using attributes that are used frequently in movie reviews. However, we avoided genre-specific attributes. The selected vignettes were evaluated best (worst) on average and showed least standard devation in evaluation.
} 


\section{Appendix 1B: Demographics, Controls and Manipulation Checks in Paper 1, Main Study}

\section{Covariates.}

Now that we have shown you a hypothetical scenario with [name], in the following we are interested in some additional information about [name] and you.

Number of Movie Recommendations. From what you can recall, how many times did [name] recommend a movie to you? (text field, only numerics)

Number of Total Recommendations. From what you can recall, how many times did [name] recommend a product to you in general? (text field, only numerics)

Description of Relationship. Please try to describe the nature of your relationship to [name] in a few keywords. (text field)

Relationship Closeness. (4 items, 7-point scales, anchors below)

How much time do you spend on joint activities with [name]? (very little time --- very much time)

How much time do you spend talking to [name]? (very little time --- very much time) To what extent do you and [name] share concerns and sorrow? (not at all --- very much) How much do you like [name]? (not at all --- very much)

Movie Expertise of Recommender. In your opinion, how knowledgeable is [name] about movies?

(7-point scale, anchors: not at all --- very much)

Duration of Relationship. How many years have you and [name] known each other? (text field, only numerics)

Age of Participant. Please state your year of birth. (text field, only numerics)

Gender of Participant. Please state your gender. (3-point scale: male, female, other)

Movie Expertise of Participant. In your opinion, how knowledgable are you about movies? (7-point scale, anchors: not at all --- very much)

\section{Appendix 1C: Screening After Data Collection in Paper 1, Main Study}

We excluded 12 participants from the analysis that did not state a real name of the hypothetical recommender in the text field. These participants wrote either comments, insults or fantasy names (e.g., "Mickey Mouse"). Please note that we nevertheless kept participants stating initials as a recommender name because of feedback that may have been done due to privacy reasons. We also excluded 18 participants that completed the questionnaire either unusually fast (lower than our test of minimum plausible session time, $n=11$ ) or especially slow (mean session time $+3 \mathrm{IQR}, \mathrm{n}=7$ ). 


\section{Appendix 2A: Scenarios, Manipulations and Phrasing of Questions in Paper 2, Study 1}

(Signaling expressions for manipulation are highlighted by gray background)

Introduction. In this study, you will be presented a scenario with a service provider. We kindly ask you to read the scenario and really try to imagine yourself as the consumer. In other words, please respond just as you would if you were actually experiencing the described situation:

Imagine that you are in the city center for Christmas shopping, but you already feel sick all day. You therefore decide to call your practitioner's office for an appointment. When you call there, the answering machine surprisingly informs you that the practice no longer exists due a move.

\section{Manipulation of Relationship Closeness.}

(Traditional Marketing Condition)

Shortly afterwards, on your way home you realize an advertisement sign that states that there is a practitioner's practice very close to your home. You now remember that practice and that it made a good impression on you from the outside. You quickly found out the phone number of the practice. Since you can't think of a more suitable practice spontaneously, you call there and make an appointment for the next day at 10:00 a.m.

\section{(Neigbor Condition)}

Shortly afterwards, on your way home you meet a neighbor with whom you don't really have much to do. When the neighbor mentions that you look sick, the conversation comes to the closed doctor's office. The neighbor spontaneously recommends a practitioner's practice with the words: "I can recommend this practice and it is very close to you. I've been there for a long time." Since you can't think of a more suitable practice, follow the neighbor's recommendation and make an appointment in the recommended practice for the next day at 10:00 am.

\section{(Friend Condition)}

Shortly afterwards, on your way home you meet a very good friend who you are very close to. When the good friend mentions that you look sick, the conversation comes to the closed doctor's office. The good friend spontaneously recommends a practitioner's practice with the words: "I can recommend this practice and it is very close to you. I've been there for a long time." Since you can't think of a more suitable practice, follow the recommendation of your good friend and make an appointment in the recommended practice for the next day at 10:00 am.

Initial Encounter Service Failure Scenario. The next morning you enter the practice on time at 9:55 a.m. You greet the receptionist and say that you have an appointment in five minutes. The woman looks at you harshly. "You are new here and then you come here just five minutes before the appointment? It must have been clear to you that you have to fill out the registration forms first and that it will take some time! I hope that, at least, you have your insurance card with you... What on earth is the matter with you?!" With this, the woman from reception hands you over the registration sheet and a ballpoint pen. You are puzzled by the abrupt address, but fill out the registration form. 


\section{Appendix 2A: Scenarios, Manipulations and Phrasing of Questions in Paper 2, Study 1 (cont.)}

Shortly afterwards it is your turn.

The attending doctor welcomes you and leads you to the treatment room. Overall, he makes a competent impression on you and takes time for the treatment. After making sure that you only have a harmless cold, he gives you a sick note for three days and says goodbye.

\section{Main Dependent Variable.}

Intent to Retain. After experiencing this situation: The next time you need to see a practitioner, e.g. because you were not feeling better a few days later, how would you behave? o I would probably retain and make an appointment in this practice.

o I would probably switch and make an appointment in another practice.

o I would not be sure what I would do.

(first two options rotated)

\section{Appendix 2B: Demographics, Controls and Manipulation Checks in Paper 2, Study 1}

\section{Covariates.}

Satisfaction with the Service. (7-point scale, anchors: do not agree at all --- fully agree)

I would have liked the described visit to the doctor.

I would be unhappy with the doctor's visit described. (r)

I would be satisfied with the doctor's visit described.

Service Failure Severity. (7-point scale, anchors: do not agree at all --- fully agree)

The harsh address would be an insignificant problem. (r)

The harsh address would be a considerable inconvenience.

The rough address would not annoy me. (r)

Reality Check. How realistic do you estimate the situation just described?

o The situation could happen like this.

o The situation could never happen like this.

Manipulation Check. Now it's all about how you got to the doctor's office in this situation.

o I came got to the practice by traditional marketing.

o I came to practice through the recommendation of a good friend.

o I came to practice on the recommendation of a neighbor.

Real-Life IESF. If you think about your real experience now. Has there been at least one situation in the past 12 months where a service directly below your expectations when you first contacted the service provider or where problems arose? Please do not only think of doctor visits, but also other services such as restaurant visits, hotel accommodation, insurance and other service providers.

o Yes, that has happened.

o No, that did not happen.

o I'm not sure.

(first two options rotated) 


\section{Appendix 2B: Demographics, Controls and Manipulation Checks in Paper 2, Study 1 (cont.)}

Intent to Complain. After the harsh address at the reception, how would you behave?

o I would probably complain.

o I would probably not complain.

o I would not be sure if I would complain.

(first two options rotated)

Age of Participant. Please state your year of birth. (text field, only numerics)

Gender of Participant. Please state your gender. (3-point scale: male, female, other)

\section{Appendix 2C: Screening After Data Collection in Paper 2, Study 1}

From 219 participants that took part in the study, 179 completed the questionnaire. We excluded 9 participants from the analysis that did not pass the manipulation check. Another 9 participants answered negatively in the reality check and were excluded. We also excluded 2 participants that completed the questionnaire unusually fast $(<90 \mathrm{sec})$. There were no participants that answered especially slow.

Appendix 2D: Logistic Regression Analysis in Paper 2, Study 1

\begin{tabular}{ccccc}
\hline & $\boldsymbol{B}$ & Wald & Sig. & $\operatorname{Exp}(\boldsymbol{B})$ \\
\hline Intercept $_{\text {Friend }^{\mathrm{a}}}$ & -.365 & .065 & .799 & 0,694 \\
Acquaintance $^{\mathrm{a}}$ & .999 & 4.448 & .035 & 2,714 \\
Satisfaction & .567 & 1.53 & .216 & 1,762 \\
Failure Severity & .701 & 9.375 & .002 & 2,015 \\
\hline
\end{tabular}

Note: $\mathrm{N}=159 ;$ Nagelkerke $\mathrm{R}^{2}=.36$.

${ }^{a}$ Reference category is Traditional Marketing. 


\section{Appendix 2E: Scenarios, Manipulations and Phrasing of Questions in Paper 2, Study 2}

(Signaling expressions for manipulation are highlighted by gray background)

Introduction. The study is about a hypothetical restaurant recommendation from a person in your social environment.

\section{Manipulation Relationship Closeness.}

(Friend Condition)

First of all, we would like to ask you to think of a very close friend of yours, where such a recommendation would be conceivable.

So please think of a real person that is

1. very close

2. where a restaurant recommendation would be realistic. Take a moment to think about it.

\section{(Acquaintance Condition)}

First of all, we would like to ask you to think of an acquaintance of yours (this does NOT mean a friend, rather a fellow student or colleague), where such a recommendation would be conceivable.

So please think of a real person that is

1. not very close

2. where a restaurant recommendation would be realistic. Take a moment to think about it.

Description Recommender Relationship. Please now indicate the relationship you have with this person. (text field)

Recommender Name. In the following scenario, we will refer several times to the person you just thought of. Please enter the first name of this person in the input field below. (text field)

Scenario of Restaurant Recommendation. You just happened to meet [name]. While you are chatting, you receive a call. As it turns out, your parents are calling because they want to visit you at your current location in the next few days. On the occasion of the reunion, your parents would like to have dinner with you. You tell your parents on the phone that you are taking care of a table in a restaurant and hang up. Since you can't think of a suitable restaurant, you ask [name] for advice. [name] recommends the little Italian "La Sicilia" in the following words: "La Sicilia is actually perfect for going out with your parents. Been there a few times. They have very delicious and authentic Italian food." You follow the recommendation of [name] and reserve a table for three people, which works without any problems.

Initial Encounter Service Failure Scenario. When your parents arrive a few days later, you go to the "La Sicilia" restaurant in the evening. The restaurant is tastefully decorated and makes a good impression, but is very crowded for the day and the time. It is almost filled to the last seat. You are glad that you have reserved in advance. The waitress accompanies you to the table and hands you the menus. After you have all made up your mind for main dishes and drinks, you place your orders. After a short time, you receive your drinks. After waiting another 30 minutes, you have already emptied your drinks, but still have not received your food. On request, when the food is going to be served, the waitress only asks you for a little more patience. Another 30 minutes pass without receiving your food. After waiting for a total of 60 minutes, the waitress finally serves your selected dishes, which, as expected, taste delicious and typically Italian. 


\section{Appendix 2E: Scenarios, Manipulations and Phrasing of Questions in Paper 2, Study 2 (cont.)}

\section{Main Dependent Variable.}

Customer Loyalty. (7-point scale, anchors: do not agree at all --- fully agree)

I would tell other people positive things about the restaurant.

I would recommend the restaurant to people who ask me for advice.

I would encourage friends and relatives to visit the restaurant.

The restaurant would be my preferred choice in the future.

I would visit the restaurant more often in the future.

\section{Mediator Variable.}

Controllability Attribution. (7-point scale, anchors: do not agree at all --- fully agree)

The long wait would have been controllable by the restaurant.

Nobody in the restaurant could have reduced the long wait.

The restaurant could have done little to reduce the waiting time.

\section{Mediator Variable.}

Stability Attribution. (7-point scale, anchors: do not agree at all --- fully agree)

It is likely that a long wait would also occur if you visit again.

I would have the feeling that such a long wait does not occur again.

I would expect the same waiting time at the restaurant in the future.

\section{Appendix 2F: Demographics, Controls and Manipulation Checks in Paper 2, Study 2}

\section{Covariates.}

Satisfaction with the Service. (7-point scale, anchors: do not agree at all --- fully agree)

I would have liked the described visit to the doctor.

I would be unhappy with the doctor's visit described. (r)

I would be satisfied with the doctor's visit described.

Service Failure Severity. (7-point scale, anchors: do not agree at all --- fully agree)

The harsh address would be an insignificant problem. (r)

The harsh address would be a considerable inconvenience.

The rough address would not annoy me. (r)

Taste Similarity. (7-point scale, anchors: do not agree at all --- fully agree)

[name] and I have a similar taste when it comes to restaurants.

[name] pays attention to the same things I do when rating a restaurant.

Conciousness Recommendation. (7-point scale, anchors: do not agree at all --- fully agree) [name] would consider carefully which restaurant he / she recommends to me.

[Name] would never give me a rash restaurant recommendation. 


\section{Appendix 2F: Demographics, Controls and Manipulation Checks in Paper 2, Study 2 (cont.)}

Reality Check. How realistic do you estimate the situation just described?

o The situation could happen like this.

o The situation could never happen like this.

Manipulation Check Waiting Time. How long was the total waiting time for your meal in the La Sicilia restaurant?

o About 15 min.

o About $45 \mathrm{~min}$

o About 60 min

Manipulation Check Filling Level. How do you rate the filling level of the restaurant?

o The restaurant was rather empty.

o The restaurant was about half full.

o The restaurant was almost completely filled.

Manipulation Check Relationship Closeness. (4 items, 7-point scales, anchors below) How much time do you spend on joint activities with [name]? (very little time --- very much time)

How much time do you spend talking to [name]? (very little time --- very much time) To what extent do you and [name] share concerns and sorrow? (not at all --- very much) How much do you like [name]? (not at all --- very much)

Age of Participant. Please state your year of birth. (text field, only numerics)

Gender of Participant. Please state your gender. (3-point scale: male, female, other)

\section{Appendix 2G: Screening After Data Collection in Paper 2, Study 2}

From 173 participants that took part in the study, 137 completed the questionnaire. We excluded 6 participants from the analysis that did not pass the manipulation checks. Another 2 participants answered negatively in the reality check and were excluded. 9 participants were excluded because manipulation was doubtable ( 2 participants stated a fantasy name for their recommender, 7 participants did not describe their relationship to the recommender or described him/her in a way that conflicted with the experimental condition). We also excluded 2 participants that completed the questionnaire unusually fast $(<150 \mathrm{sec})$. There were no participants that answered especially slow. 


\section{Appendix 2H: Mediation Analysis in Paper 2, Study 2}

Hayes Model 4 Parallel Mediation, 5000 Bootstraps

$\mathrm{X}$ : Relationship Closeness

Mediator 1: Controllability Attribution

Mediator 2: Stability Attribution

Y: Customer Loyalty

Covariates: Satisfaction, Severity

Outcome Variable: Controllability Attribution

\begin{tabular}{ccccc}
\hline & Coefficient & SE & $\boldsymbol{t}$ & $\boldsymbol{p}$ \\
\hline Constant & 2.300 & .751 & 3.060 & .003 \\
Relationship Closeness & .016 & .055 & .288 & .774 \\
Satisfaction & .060 & .091 & .664 & .508 \\
Severity & .461 & .901 & 5.120 & .000 \\
\hline
\end{tabular}

Outcome Variable: Stability Attribution

\begin{tabular}{ccccc}
\hline & Coefficient & SE & $\boldsymbol{t}$ & $\boldsymbol{p}$ \\
\hline Constant & 3.410 & .985 & 3.462 & .000 \\
Relationship Closeness & -.198 & .072 & -2.771 & .007 \\
Satisfaction & .159 & .119 & 1.335 & .185 \\
Severity & .322 & .118 & 2.722 & .008 \\
\hline
\end{tabular}

Outcome Variable: Customer Loyalty

\begin{tabular}{ccccc}
\hline & Coefficient & SE & $\boldsymbol{t}$ & $\boldsymbol{p}$ \\
\hline Constant & 4.391 & .700 & 6.270 & .000 \\
Relationship Closeness & .103 & .049 & 2.073 & .041 \\
Controllability Att. & -.021 & .086 & -.238 & .812 \\
Stability Att. & -.363 & .660 & -5.506 & .000 \\
Satisfaction & .418 & .080 & 5.234 & .000 \\
Severity & -.135 & .088 & -1.542 & .126 \\
\hline & Direct Effect of X on Y & & \\
\hline & Effect & SE & $\boldsymbol{t}$ & $\boldsymbol{p}$ \\
\hline & .103 & .049 & 2.073 & .040
\end{tabular}

Indirect Effects of X on Y

\begin{tabular}{ccccc}
\hline & Effect & BootSE & BootLLCI & BootULCI \\
\hline TOTAL & .072 & .030 & .017 & .136 \\
Controllability Att. & -.000 & .005 & -.011 & .012 \\
Stability Att. & .072 & .030 & .021 & .134 \\
\hline
\end{tabular}




\section{Appendix 3: Suggestions for reporting PROCESS results (based on the data examples)}

\section{Simple Mediation}

We used PROCESS model 4 (Hayes 2013) to test the proposed mediation. The data is consistent with the claim that $A_{A d}$ impacts $A_{B r a n d}$, which in turn increases $P I(\mathrm{~b}=.31 ; 95 \% \mathrm{CI}=.16$ to .49). The mediation partially explains the effect of $A_{A d}$ on $P I$; in addition, $A_{A d}$ influences $P I$ independently from the proposed mechanism $(\mathrm{b}=.15, p=.047)$. Hence, we infer complementary partial mediation (Zhao et al. 2010).

The reader may refer to Schrift and Amar (2015) and Siddiqui et al. (2017) for further examples of reporting simple mediation results yielded from PROCESS in the marketing literature.

\section{Parallel Mediation}

We used PROCESS model 4 (Hayes 2013) to test the proposed mediations. Overall, we could establish a mediation of both proposed mediators, resulting in a significant mediation from $A_{A d}$ to $P I$ through $A_{\text {Brand }}(\mathrm{b}=.26 ; 95 \% \mathrm{CI}: .14$ to .43 ) and recall $(\mathrm{b}=.08 ; 95 \% \mathrm{CI}$ : .02 to .17$)$. The proposed mediation through $A_{B r a n d}$ is significantly stronger than the one through product recall $\left(\Delta_{\mathrm{b}}=.17 ; 95 \% \mathrm{CI}: .01\right.$ to .36$)$. There is an insignificant direct effect of $A_{A d}$ on $P I(\mathrm{~b}=.12, p=$ .075). Taken together, the findings indicate full parallel mediation.

The reader may refer to Hur et al. (2015) and Winterich and Zhang (2014) for further examples of reporting parallel mediation results yielded from PROCESS in the marketing literature.

\section{Serial Mediation}

Using PROCESS model 6 (Hayes 2013) we could establish a serial mediation from $A_{A d}$ through $A_{\text {Brand }}$ through recall to PI ( $\mathrm{b}=.05 ; 95 \% \mathrm{CI}$ : .02 to .11$)$. In addition, $A_{A d}$ had an indirect effect on $P I$ through $A_{\text {Brand }}(\mathrm{b}=.25,95 \% \mathrm{CI} .13$ to .41$)$ but not through recall $(\mathrm{b}=.03,95 \% \mathrm{CI}-.04$ to .12). Finally, there is no direct effect of $A_{A d}$ on $P I(\mathrm{~b}=.12, p=.075)$, indicating full serial mediation.

The reader may refer to Hur et al. (2015) and Winterich and Zhang (2014) for further examples of reporting serial mediation results yielded from PROCESS in the marketing literature.

\section{Moderated Mediation}

We used PROCESS model 7 (Hayes 2013) to test the proposed moderated mediation. Overall, we could establish a moderated mediation from $A_{A d}$ through $A_{B r a n d}$ to PI, dependent on elaboration mode (index of moderated mediation: .31; $95 \% \mathrm{CI}$ : .06 to .63). While for the low elaboration group there is a significant indirect effect of $A_{A d}$ on $P I$ through $A_{B r a n d}(\mathrm{~b}=.45,95 \%$ CI .24 to .66), the effect disappears when elaboration is high ( $b=.14,95 \% \mathrm{CI}-.07$ to .34$)$.

The reader may refer to Blanchard et al. (2016) and Hur et al. (2015) for further examples of reporting moderated mediation results yielded from PROCESS in the marketing. 
This page is intentionally left blank. 


\section{References}

Alexander, M. J., Jaakkola, E., \& Hollebeek, L. D. (2018). Zooming Out: Actor Engagement Beyond the Dyadic. Journal of Service Management, 29(3), 333-351.

Anderson, J., \& Gerbing, D. W. (1988). Structural Equation Modeling in Practice: A Review and Recommended Two-Step Approach. Psychological Bulletin, 103(3), 411-423.

Anderson. (1981). Foundations of Information Integration Theory. New York: Academic Press.

Arbuckle, J. L. (2016). IBM SPSS Amos 24 User's Guide. Chicago: Amos Development Corporation.

Arndt, J. (1967). Word of Mouth Advertising: A Review of the Literature: Advertising Research Foundation.

Aroian, L. A. (1947). The Probability Function of the Product of Two Normally Distributed Variables. The Annals of Mathematical Statistics, 265-271.

Bagozzi, R. P., \& Yi, Y. (1988). On the Evaluation of Structural Equation Models. Journal of the Academy of Marketing Science, 16(1), 74-94.

Bansal, H. S., \& Voyer, P. A. (2000). Word-of-Mouth Processes within a Services Purchase Decision Context. Journal of Service Research, 3(2), 166-177.

Baron, R. M., \& Kenny, D. A. (1986). The Moderator-Mediator Variable Distinction in Social Psychological Research: Conceptual, Strategic, and Statistical Considerations. Journal of Personality and Social Psychology, 51(6), 1173-1182.

Baxter, L. A., \& Bullis, C. (1986). Turning Points in Developing Romantic Relationships. Human Communication Research, 12(4), 469-493. 
Berger, J. (2014). Word of Mouth and Interpersonal Communication: A Review and Directions for Future Research. Journal of Consumer Psychology, 24(4), 586-607. Berry, L. L. (1995). Relationship Marketing of Services: Growing Interest, Emerging Perspectives. Journal of the Academy of Marketing Science, 23(4), 236-245.

Berry, L. L., \& Parasuraman, Anantharanthan. (2004). Marketing Services: Competing Through Quality. New York: Simon and Schuster.

Bitner, M. J., Booms, B. H., \& Tetreault, M. S. (1990). The Service Encounter: Diagnosing Favorable and Unfavorable Incidents. Journal of Marketing, 54(1), 71-84.

Blanchard, S. J., Carlson, K. A., \& Hyodo, J. D. (2016). The Favor Request Effect: Requesting a Favor from Consumers to Seal the Deal. Journal of Consumer Research, 42(6), 985-1001.

Bollen, K. A. (1989). A New Incremental Fit Index for General Structural Equation Models. Sociological Methods \& Research, 17(3), 303-316.

Bollen, K. A., \& Stine, R. (1990). Direct and Indirect Effects: Classical and Bootstrap Estimates of Variability. Sociological Methodology, 20(1), 15-140.

Bolton, R. N. (1998). A Dynamic Model of the Duration of the Customer's Relationship with a Continuous Service Provider: The Role of Satisfaction. Marketing Science, 17(1), 45-65. Bonifield, C., \& Cole, C. (2007). Affective Responses to Service Failure: Anger, Regret, and Retaliatory versus Conciliatory Responses. Marketing Letters, 18(1), 85-99.

Boulding, W., Kalra, A., Staelin, R., \& Zeithaml, V. A. (1993). A Dynamic Process Model of Service Quality: From Expectations to Behavioral Intentions. Journal of Marketing Research, 30(1), 7-27. 
Brodie, R. J., Fehrer, J. A., Jaakkola, E., \& Conduit, J. (2019). Actor Engagement in Networks: Defining the Conceptual Domain. Journal of Service Research, 22(2), 173-188.

Brown, \& Reingen, P. H. (1987). Social Ties and Word-of-Mouth Referral Behavior. Journal of Consumer Research, 14(3), 350-362.

Brown, S., \& Stayman, D. M. (1992). Antecedents and Consequences of Attitude Toward the Ad: A Meta-Analysis. Journal of Consumer Research, 19(1), 34-51.

Cavanaugh, L. A. (2016). Consumer Behavior in Close Relationships. Current Opinion in Psychology, 10, 101-106.

Cheng, S., \& Lam, T. (2008). The Role of the Customer-Seller Relationship in the Intention of the Customer to Complain: A Study of Chinese Restaurateurs. International Journal of Hospitality Management, 27(4), 552-562.

Cheung, G. W., \& Lau, R. S. (2008). Testing Mediation and Suppression Effects of Latent Variables Bootstrapping With Structural Equation Models. Organizational Research Methods, 11(2), 296-325.

Cohen, G., Aronson, J., \& Steele, C. M. (2000). When Beliefs Yield to Evidence: Reducing Biased Evaluation by Affirming the Self. Personality and Social Psychology Bulletin, 26(9), 1151-1164.

Cohen, J. (1990). Things I Have Learned (So Far). American Psychologist, 45(12), 13041312.

Creedon, P. S., \& Hayes, A. F. (2015). Small Sample Mediation Analysis: How Far Can We Push the Bootstrap. Poster presented at Ohio State University, Columbus. 
Danner, D., Hagemann, D., \& Fiedler, K. (2015). Mediation Analysis with Structural Equation Models: Combining Theory, Design, and Statistics. European Journal of Social Psychology, 45(4), 460-481.

Denrell, J. (2005). Why Most People Disapprove of Me: Experience Sampling in Impression Formation. Psychological Review, 112(4), 951-978.

DeWitt, T., \& Brady, M. K. (2003). Rethinking Service Recovery Strategies. Journal of Service Research, 6(2), 193-207.

Duhan, D. F., Johnson, S. D., Wilcox, J. B., \& Harrell, G. D. (1997). Influences on Consumer Use of Word-of-Mouth Recommendation Sources. Journal of the Academy of Marketing Science, 25(4), 283-295.

Efron, B. (1982). The Jackknife, the Bootstrap and Other Resampling Plans. Stanford: SIAM.

Efron, B. (1987). Better Bootstrap Confidence Intervals. Journal of the American Statistical Association, 82(397), 171-185.

Efron, B., \& Tibshirani, R. J. (1993). An Introduction to the Bootstrap. New York: Chapman \& Hall.

Eggleston, C. M., Wilson, T. D., Lee, M., \& Gilbert, D. T. (2015). Predicting What We Will Like: Asking a Stranger Can Be as Good as Asking a Friend. Organizational Behavior and Human Decision Processes, 128, 1-10.

Feick, L., \& Higie, R. A. (1992). The Effects of Preference Heterogeneity and Source Characteristics on Ad Processing and Judgements about endorsers. Journal of Advertising, 21(2), 9-24.

Fiedler, K., Schott, M., \& Meiser, T. (2011). What Mediation Analysis Can (Not) Do. Journal of Experimental Social Psychology, 47(6), 1231-1236. 
Fitzsimons, G. J. (2008). Death to Dichotomizing. Journal of Consumer Research, 35(1), 5-8.

Fritz, M. S., \& MacKinnon, D. P. (2007). Required Sample Size to Detect the Mediated Effect. Psychological Science, 18(3), 233-239.

Germann, F., Grewal, R., Ross, W. T., \& Srivastava, R. K. (2014). Product Recalls and the Moderating Role of Brand Commitment. Marketing Letters, 25(2), 179-191.

Gershoff, A. D., Broniarczyk, S. M., \& West, P. (2001). Recommendation or Evaluation? Task Sensitivity in Information Source Selection. Journal of Consumer Research, 28(3), $418-438$.

Gershoff, A. D., \& Johar, G. V. (2006). Do You Know Me? Consumer Calibration of Friends' Knowledge. Journal of Consumer Research, 32(4), 496-503.

Gershoff, A. D., Mukherjee, A., \& Mukhopadhyay, A. (2003). Consumer Acceptance of Online Agent Advice: Extremity and Positivity Effects. Journal of Consumer Psychology, 13(1\&2), 161-170.

Gershoff, A. D., Mukherjee, A., \& Mukhopadhyay, A. (2007). Few Ways to Love, But Many Ways to Hate: Attribute Ambiguity and the Positivity Effect in Agent Evaluation. Journal of Consumer Research, 33(4), 499-505.

Goodman, L. A. (1960). On the Exact Variance of Products. Journal of the American Statistical Association, 55(292), 708-713.

Granovetter, M. S. (1973). The Strength of Weak Ties. American Journal of Sociology, 13601380.

Guevarra, D. A., \& Howell, R. T. (2015). To Have in Order to Do: Exploring the Effects of Consuming Experiential Products on Well-Being. Journal of Consumer Psychology, 25(1), $28-41$. 
Harmeling, C. M., Palmatier, R. W., Houston, M. B., Arnold, M. J., \& Samaha, S. A. (2015). Transformational Relationship Events. Journal of Marketing, 79(5), 39-62.

Harvey, N., \& Fischer, I. (1997). Taking Advice: Accepting Help, Improving Judgment, and Sharing Responsibility. Organizational Behavior and Human Decision Processes, 70(2), $117-133$.

Hayes, A. F. (2009). Beyond Baron and Kenny: Statistical Mediation Analysis in the New Millennium. Communication Monographs, 76(4), 408-420.

Hayes, A. F. (2013). Introduction to Mediation, Moderation, and Conditional Process Analysis: A Regression-Based Approach. New York: Guilford Press.

Hayes, A. F. (2015). An Index and Test of Linear Moderated Mediation. Multivariate Behavioral Research, 50(1), 1-22.

Hayes, A. F. (2017). The PROCESS Macro for SPSS and SAS. Retrieved from http://processmacro.org/index.html

Hayes, A. F. (2018). Introduction to Mediation, Moderation, and Conditional Process Analysis: A Regression-Based Approach (2nd ed.). New York: Guilford.

Hayes, A. F., \& Preacher, K. J. (2010). Quantifying and Testing Indirect Effects in Simple Mediation Models When the Constituent Paths are Nonlinear. Multivariate Behavioral Research, 45(4), 627-660.

Hayes, A. F., \& Preacher, K. J. (2014). Statistical Mediation Analysis with a Multicategorical Independent Variable. British Journal of Mathematical and Statistical Psychology, 67(3), 451-470. 
Hayes, A. F., \& Scharkow, M. (2013). The Relative Trustworthiness of Inferential Tests of the Indirect Effect in Statistical Mediation Analysis: Does Method Really Matter? Psychological Science, 24(10), 1918-1927.

Heath, T. B., Chatterjee, S., Basuroy, S., Hennig-Thurau, T., \& Kocher, B. (2015). Innovation Sequences Over Iterated Offerings: A Relative Innovation, Comfort, and Stimulation Framework of Consumer Responses. Journal of Marketing, 79(6), 71-93.

Heide, J. B. (1994). Interorganizational Governance in Marketing Channels. Journal of Marketing, 58(1), 71-85.

Heilman, C. M., Bowman, D., \& Wright, G. P. (2000). The Evolution of Brand Preferences and Choice Behaviors of Consumers New to a Market. Journal of Marketing Research, $37(2), 139-155$.

Hess, R. L. (2008). The Impact of Firm Reputation and Failure Severity on Customers' Responses to Service Failures. Journal of Services Marketing, 22(5), 385-398.

Hess, R. L., Ganesan, S., \& Klein, N. (2003). Service Failure and Recovery: The Impact of Relationship Factors on Customer Satisfaction. Journal of the Academy of Marketing Science, 31(2), 127-145.

Hogarth, R. M., \& Einhorn, H. J. (1992). Order Effects in Belief Updating: The BeliefAdjustment Model. Cognitive Psychology, 24(1), 1-55.

Hu, L.-t., \& Bentler, P. M. (1998). Fit Indices in Covariance Structure Modeling: Sensitivity to Underparameterized Model Misspecification. Psychological Methods, 3(4), 424-453.

Hui, M. K., \& Toffoli, R. (2002). Perceived Control and Consumer Attribution for the Service Encounter. Journal of Applied Social Psychology, 32(9), 1825-1844. 
Hur, J. D., Koo, M., \& Hofmann, W. (2015). When Temptations Come Alive: How Anthropomorphism Undermines Self-Control. Journal of Consumer Research, 42(2), 340358.

Iacobucci, D. (2005). From the Editor: On p-Values. Journal of Consumer Research, 32(1), $6-11$.

Iacobucci, D. (2008). Mediation Analysis. Thousand Oaks: Sage.

Iacobucci, D. (2009). Everything You Always Wanted to Know about SEM (Structural Equations Modeling) But Were Afraid to Ask. Journal of Consumer Psychology, 19(4), $673-680$.

Iacobucci, D. (2010). Structural Equations Modeling: Fit Indices, Sample Size, and Advanced Topics. Journal of Consumer Psychology, 20(1), 90-98.

Iacobucci, D., Saldanha, N., \& Deng, X. (2007). A Meditation on Mediation: Evidence That Structural Equations Models Perform Better Than Regressions. Journal of Consumer Psychology, 17(2), 140-154.

James, L. R., \& Brett, J. M. (1984). Mediators, Moderators, and Tests for Mediation. Journal of Applied Psychology, 69(2), 307-321.

Journal of Consumer Research. (2017). JCR Style Sheet. Retrieved from http://www.ejcr.org/newstylesheet.pdf

Judd, C. M., \& Kenny, D. A. (1981). Process Analysis: Estimating Mediation in Treatment Evaluations. Evaluation Review, 5(5), 602-619.

Jussim, L., \& Osgood, D. W. (1989). Influence and Similarity Among Friends: An Integrative Model Applied to Incarcerated Adolescents. Social Psychology Quarterly, 98-112. 
Kalamas, M., Laroche, M., \& Makdessian, L. (2008). Reaching the Boiling Point: Consumers' Negative Affective Reactions to Firm-Attributed Service Failures. Journal of Business Research, 61(8), 813-824.

Keaveney, S. M. (1995). Customer Switching Behavior in Service Industries: An Exploratory Study. Journal of Marketing, 59(2), 71-82.

Kenny, D. A. (2008). Reflections on Mediation. Organizational Research Methods, 11(2), $353-358$.

Kenny, D. A., \& Acitelli, L. K. (2001). Accuracy and Bias in the Perception of the Partner in a Close Relationship. Journal of Personality and Social Psychology, 80(3), 439-448.

Kisbu-Sakarya, Y., MacKinnon, D. P., \& Miočević, M. (2014). The Distribution of the Product Explains Normal Theory Mediation Confidence Interval Estimation. Multivariate Behavioral Research, 49(3), 261-268.

Klein, W., \& Kunda, Z. (1992). Motivated Person Perception: Constructing Justifications for Desired Beliefs. Journal of Experimental Social Psychology, 28(2), 145-168.

Koopman, J., Howe, M., Hollenbeck, J. R., \& Sin, H.-P. (2015). Small Sample Mediation Testing: Misplaced Confidence in Bootstrapped Confidence Intervals. Journal of Applied Psychology, 100(1), 194-202.

Kruglanski, A. W., \& Freund, T. (1983). The Freezing and Unfreezing of Lay-Inferences: Effects on Impressional Primacy, Ethnic Stereotyping, and Numerical Anchoring. Journal of Experimental Social Psychology, 19(5), 448-468.

Kumar, V., Aksoy, L., Donkers, B., Venkatesan, R., Wiesel, T., \& Tillmanns, S. (2010). Undervalued or Overvalued Customers: Capturing Total Customer Engagement Value. Journal of Service Research, 13(3), 297-310. 
Kunda, Z., \& Thagard, P. (1996). Forming Impressions from Stereotypes, Traits, and Behaviors: A Parallel-Constraint-Satisfaction Theory. Psychological Review, 103(2), 284308.

Lerouge, D., \& Warlop, L. (2006). Why It Is So Hard to Predict Our Partner's Product Preferences: The Effect of Target Familiarity on Prediction Accuracy. Journal of Consumer Research, 33(3), 393-402.

Locke, K. D., Craig, T., Baik, K.-D., \& Gohil, K. (2012). Binds and Bounds of Communion: Effects of Interpersonal Values on Assumed Similarity of Self and Others. Journal of Personality and Social Psychology, 103(5), 879-897.

Lord, C. G., \& Taylor, C. A. (2009). Biased Assimilation: Effects of Assumptions and Expectations on the Interpretation of New Evidence. Social and Personality Psychology Compass, 3(5), 827-841.

Macho, S., \& Ledermann, T. (2011). Estimating, Testing, and Comparing Specific Effects in Structural Equation Models: The Phantom Model Approach. Psychological Methods, 16(1), 34-43.

MacKenzie, S. B., Lutz, R. J., \& Belch, G. E. (1986). The Role of Attitude Toward the Ad as a Mediator of Advertising Effectiveness: A Test of Competing Explanations. Journal of Marketing Research, 23(2), 130-143.

MacKenzie, S. B., \& Spreng, R. A. (1992). How Does Motivation Moderate the Impact of Central and Peripheral Processing on Brand Attitudes and Intentions? Journal of Consumer Research, 18(4), 519-529.

MacKinnon, D. P., Krull, J. L., \& Lockwood, C. M. (2000). Equivalence of the Mediation, Confounding and Suppression Effect. Prevention Science, 1(4), 173-181. 
MacKinnon, D. P., Lockwood, C. M., Hoffman, J. M., West, S., \& Sheets, V. (2002). A Comparison of Methods to Test Mediation and Other Intervening Variable Effects. Psychological Methods, 7(1), 83-104.

MacKinnon, D. P., Warsi, G., \& Dwyer, J. H. (1995). A Simulation Study of Mediated Effect Measures. Multivariate Behavioral Research, 30(1), 41.

Matos, C. A. de, Henrique, J. L., \& Rossi, C. A. V. (2007). Service Recovery Paradox: A Meta-Analysis. Journal of Service Research, 10(1), 60-77.

Mattila, A. S. (2001). The impact of Relationship Type on Customer Loyalty in a Context of Service Failures. Journal of Service Research, 4(2), 91-101.

Mattila, A. S. (2003). The Impact of Cognitive Inertia on Postconsumption Evaluation Processes. Journal of the Academy of Marketing Science, 31(3), 287-299.

Mattila, A. S. (2004). The Impact of Service Failures on Customer Loyalty. International Journal of Service Industry Management, 15(2), 134-149.

McCollough, M. A., Berry, L. L., \& Yadav, M. S. (2000). An Empirical Investigation of Customer Satisfaction After Service Failure and Recovery. Journal of Service Research, 3(2), 121-137.

Mittal, V., Huppertz, J., \& Khare, A. (2008). Customer Complaining: The Role of Tie Strength and Information Control. Journal of Retailing, 84(2), 195-204.

Muller, D., Judd, C. M., \& Yzerbyt, V. Y. (2005). When Moderation is Mediated and Mediation is Moderated. Journal of Personality and Social Psychology, 89(6), 852-863.

Muthen, L., \& Muthen, B. (1998-2015). Mplus User's Guide (7th ed.). Los Angeles: Muthén \& Muthén.

Nielsen. (2015). Nielsen Global Trust in Advertising Survey. 
Nitzan, I., \& Libai, B. (2011). Social Effects on Customer Retention. Journal of Marketing, 75(6), 24-38.

Oliver, R. L., \& Swan, J. E. (1989). Consumer Perceptions of Interpersonal Equity and Satisfaction in Transactions: A Field Survey Approach. Journal of Marketing, 53(2), 2135.

Preacher, K. J. (2015). Advances in Mediation Analysis: A Survey and Synthesis of New Developments. Annual Review of Psychology, 66, 825-852.

Preacher, K. J., \& Hayes, A. F. (2004). SPSS and SAS Procedures for Estimating Indirect Effects in Simple Mediation Models. Behavior Research Methods, 36(4), 717-731.

Preacher, K. J., \& Hayes, A. F. (2008). Asymptotic and Resampling Strategies for Assessing and Comparing Indirect Effects in Multiple Mediator Models. Behavior Research Methods, 40(3), 879-891.

Preacher, K. J., Rucker, D. D., \& Hayes, A. F. (2007). Addressing Moderated Mediation Hypotheses: Theory, Methods, and Prescriptions. Multivariate Behavioral Research, 42(1), $185-227$.

Rosen, D. L., \& Olshavsky, R. W. (1987). A Protocol Analysis of Brand Choice Strategies Involving Recommendations. Journal of Consumer Research, 14(3), 440-444.

Rucker, D. D., Preacher, K. J., Tormala, Z. L., \& Petty, R. E. (2011). Mediation Analysis in Social Psychology: Current Practices and New Recommendations. Social and Personality Psychology Compass, 5(6), 359-371.

Sajtos, L., Brodie, R. J., \& Whittome, J. (2010). Impact of Service Failure: The Protective Layer of Customer Relationships. Journal of Service Research, 13(2), 216-229. 
Schmitt, P., Skiera, B., \& van den Bulte, C. (2011). Referral Programs and Customer Value. Journal of Marketing, 75(1), 46-59.

Scott, C. A. (1977). Modifying Socially-Conscious Behavior: The Foot-in-the-Door Technique. Journal of Consumer Research, 4(3), 156-164.

Shrout, P. E., \& Bolger, N. (2002). Mediation in Experimental and Nonexperimental Studies: New Procedures and Recommendations. Psychological Methods, 7(4), 422-445.

Simpson, J. A., Griskevicius, V., \& Rothman, A. J. (2012). Consumer Decisions in Relationships. Journal of Consumer Psychology, 22(3), 304-314.

Singh, J. (1990). Voice, Exit, and Negative Word-of-Mouth Behaviors: An Investigation Across Three Service Categories. Journal of the Academy of Marketing Science, 18(1), 115.

Smith, A. K., \& Bolton, R. N. (1998). An Experimental Investigation of Customer Reactions to Service Failure and Recovery Encounters. Journal of Service Research, 1(1), 65-81.

Smith, A. K., Bolton, R. N., \& Wagner, J. (1999). A Model of Customer Satisfaction With Service Encounters Involving Failure and Recovery. Journal of Marketing Research, $36(3), 356-372$.

Sobel, M. E. (1982). Asymptotic Confidence Intervals for Indirect Effects in Structural Equation Models. Sociological Methodology, 13, 290-312.

Sobel, M. E. (1986). Some New Results on Indirect Effects and Their Standard Errors in Covariance Structure Models. Sociological Methodology, 16, 159-186.

Spiller, S. A., Fitzsimons, G. J., Lynch, J. G., \& McClelland, G. H. (2013). Spotlights, Floodlights, and the Magic Number Zero: Simple Effects Tests in Moderated Regression. Journal of Marketing Research, 50(2), 277-288. 
Stelzl, I. (1986). Changing a Causal Hypothesis Without Changing the Fit: Some Rules for Generating Equivalent Path Models. Multivariate Behavioral Research, 21(3), 309-331.

Stone, C. A., \& Sobel, M. E. (1990). The Robustness of Estimates of Total Indirect Effects in Covariance Structure Models Estimated By Maximum. Psychometrika, 55(2), 337-352.

Swann, W. B., \& Gill, M. J. (1997). Confidence and Accuracy in Person Perception: Do We Know What We Think We Know About Our Relationship Partners? Journal of Personality and Social Psychology, 73(4), 747-757.

Thomas, J. S. (2001). A Methodology for Linking Customer Acquisition to Customer Retention. Journal of Marketing Research, 38(2), 262-268.

Trusov, M., Bucklin, R. E., \& Pauwels, K. (2009). Effects of Word-of-Mouth Versus Traditional Marketing: Findings From an Internet Social Networking Site. Journal of Marketing, 73(5), 90-102.

Tubbs, R. M., Gaeth, G. J., Levin, I. P., \& van Osdol, L. A. (1993). Order Effects in Belief Updating With Consistent and Inconsistent Evidence. Journal of Behavioral Decision Making, 6(4), 257-269.

Tuk, M. A., Verlegh, P. W.J., Smidts, A., \& Wigboldus, D. H.J. (2019). You and I have nothing in common: The role of dissimilarity in interpersonal influence. Organizational Behavior and Human Decision Processes, 151, 49-60.

Van den Bulte, C., Bayer, E., Skiera, B., \& Schmitt, P. (2018). How Customer Referral Programs Turn Social Capital Into Economic Capital. Journal of Marketing Research, $55(1), 132-146$.

Van Raaij, W. F., \& Pruyn, A. T.H. (1998). Customer Control and Evaluation of Service Validity and Reliability. Psychology and Marketing, 15(8), 811-832. 
Van Vaerenbergh, Y., Orsingher, Chiara, Vermeir, I., \& Larivière, B. (2014). A MetaAnalysis of Relationships Linking Service Failure Attributions to Customer Outcomes. Journal of Service Research, 17(4), 381-398.

Vázquez-Casielles, R., del Río-Lanza, A. B., \& Díaz-Martín, A. M. (2007). Quality of Past Performance: Impact on consumers' Responses to Service Failure. Marketing Letters, $18(4), 249-264$.

Villanueva, J., Yoo, S., \& Hanssens, D. M. (2008). The Impact of Marketing-Induced Versus Word-of-Mouth Customer Acquisition on Customer Equity Growth. Journal of Marketing Research, 45(1), 48-59.

Voorhees, C. M., Fombelle, P. W., Gregoire, Y., Bone, S., Gustafsson, A., Sousa, R., \& Walkowiak, T. (2017). Service Encounters, Experiences and the Customer Journey: Defining the Field and a Call to Expand Our Lens. Journal of Business Research, 79, 269280.

Weiner, B. (1985). An Attributional Theory of Achievement Motivation and Emotion. Psychological Review, 92(4), 548-573.

Weiner, B. (2000). Attributional Thoughts About Consumer Behavior. Journal of Consumer Research, 27(3), 382-387.

White, T. B. (2005). Consumer Trust and Advice Acceptance: The Moderating Roles of Benevolence, Expertise, and Negative Emotions. Journal of Consumer Psychology, 15(2), $141-148$.

Winterich, K. P., \& Zhang, Y. (2014). Accepting Inequality Deters Responsibility: How Power Distance Decreases Charitable Behavior. Journal of Consumer Research, 41(2), 274-293. 
Wirtz, J., Orsingher, C., Chew, P., \& Tambyah, S. K. (2013). The Role of Metaperception on the Effectiveness of Referral Reward Programs. Journal of Service Research, 16(1), 8298.

Yaniv, I. (2004). Receiving Other People's Advice: Influence and Benefit. Organizational Behavior and Human Decision Processes, 93(1), 1-13.

Yaniv, I., Choshen-Hillel, S., \& Milyavsky, M. (2011). Receiving Advice on Matters of Taste: Similarity, Majority Influence, and Taste Discrimination. Organizational Behavior and Human Decision Processes, 115(1), 111-120.

Yaniv, I., \& Kleinberger, E. (2000). Advice Taking in Decision Making: Egocentric Discounting and Reputation Formation. Organizational Behavior and Human Decision Processes, 83(2), 260-281.

Zeithaml, V. A., Berry, L. L., \& Parasuraman, A. (1996). The Behavioral Consequences of Service Quality. Journal of Marketing, 60(2), 31-46.

Zhao, X., Lynch, J. G., \& Chen, Q. (2010). Reconsidering Baron and Kenny: Myths and Truths About Mediation Analysis. Journal of Consumer Research, 37(2), 197-206. 


\section{Eidesstattliche Erklärung}

Ich versichere,

1. dass ich die eingereichte Dissertation When Friends Give Bad Advice - Analyzing Response to Recommendation Performance of Close Others selbstständig angefertigt habe und nicht die Hilfe Dritter in einer dem Prüfungsrecht und wissenschaftlicher Redlichkeit widersprechenden Weise in Anspruch genommen habe,

2. dass ich das Prüfungsrecht einschließlich der wissenschaftlichen Redlichkeit - hierzu gehört die strikte Beachtung des Zitiergebots, so dass die Übernahme fremden Gedankenguts in der Dissertation deutlich gekennzeichnet ist - beachtet habe,

3. dass beim vorliegenden Promotionsverfahren kein Vermittler gegen Entgelt eingeschaltet worden ist sowie im Zusammenhang mit dem Promotionsverfahren und seiner Vorbereitung kein Entgelt gezahlt oder entgeltgleiche Leistungen erbracht worden sind - keine Dienste unentgeltlich in Anspruch genommen wurden, die dem Sinn und Zweck eines Prüfungsverfahrens widersprechen,

4. dass ich eine entsprechende Promotion nicht anderweitig beantragt und hierbei die eingereichte Dissertation oder Teile daraus vorgelegt habe. Mir ist bekannt, dass Unwahrheiten hinsichtlich der vorstehenden Versicherung die Zulassung zur Promotionsprüfung ausschließen und im Falle eines späteren Bekanntwerdens die Promotionsprüfung für ungültig erklärt werden oder der Doktorgrad aberkannt werden kann.

Göttingen, 03.04.2020

Carsten Leo Demming 